\title{
GENERAL LOGICAL METATHEOREMS FOR FUNCTIONAL ANALYSIS
}

\author{
PHILIPP GERHARDY AND ULRICH KOHLENBACH
}

\begin{abstract}
In this paper we prove general logical metatheorems which state that for large classes of theorems and proofs in (nonlinear) functional analysis it is possible to extract from the proofs effective bounds which depend only on very sparse local bounds on certain parameters. This means that the bounds are uniform for all parameters meeting these weak local boundedness conditions. The results vastly generalize related theorems due to the second author where the global boundedness of the underlying metric space (resp. a convex subset of a normed space) was assumed. Our results treat general classes of spaces such as metric, hyperbolic, CAT(0), normed, uniformly convex and inner product spaces and classes of functions such as nonexpansive, Hölder-Lipschitz, uniformly continuous, bounded and weakly quasinonexpansive ones. We give several applications in the area of metric fixed point theory. In particular, we show that the uniformities observed in a number of recently found effective bounds (by proof theoretic analysis) can be seen as instances of our general logical results.
\end{abstract}

\section{INTRODUCTION}

In [25], the second author established - as part of a general project of applied proof theory - logical metatheorems which guarantee a priorily the extractability of effective uniform bounds from large classes of proofs in functional analysis. 'Uniformity' here refers to the independence of the bounds from parameters ranging over compact subspaces (in the case of concrete Polish metric spaces) as well as abstract bounded (not necessarily compact!) metric spaces or bounded convex subsets of hyperbolic, CAT(0), normed, uniformly convex or inner product spaces. By 'abstract' spaces we mean that the proofs only use the general axioms for e.g. metric or hyperbolic spaces. If these axioms have a strong uniformity built in (as in the classes just mentioned), then this property also prevails for theorems proved in strong theories based on these axioms. The metatheorems were derived using a monotone proof interpretation, namely an extension of Gödel's so-called functional interpretation combined with a novel form of majorizability over function spaces of arbitrary types. The theorems were applied to results in metric fixed point theory to explain the extractability of strong uniform bounds that had been observed previously in several concrete cases $([21,23,28])$ as well as to predict new

Received by the editors March 17, 2006.

2000 Mathematics Subject Classification. Primary 03F10, 03F35, 47H09, 47H10. 
such bounds which subsequently could, indeed, be found following the extraction algorithm provided by monotone functional interpretation $([26,24])$.

In the concrete applications it usually turned out that instead of the assumption of the whole space or some convex subset being bounded only some sparse local boundedness conditions were actually needed. This observation was the starting point of the present paper which establishes far reaching extensions of the results from [25] to unbounded spaces which guarantee effective uniform bounds under exactly such limited local boundedness assumptions. As we will show below, in most applications our new metatheorems completely close the gap which was left between the conclusions predicted by the old metatheorems and the general form of actual bounds constructed in the case studies. In particular, we now for the first time can explain a quantitative version of a well-known theorem of Borwein, Reich and Shafrir [2] on Krasnoselski-Mann iterations of nonexpansive mappings in unbounded hyperbolic spaces, which was found in [28], as an instance of the new metatheorems. The proofs still use a combination of functional interpretation and majorization, but this time in a much more subtle way: both the functional interpretation as well as the majorization relation to be applied are parametrized by a point $a$ of the space $X$ in question. In the applications we will be able to achieve by a suitable choice of $a$ (which in turn depends on the parameters of the problem) that the object constructed by the $a$-functional interpretation can be $a$-majorized by a term which no longer depends on $a$ (nor the parameters involving the space $X$ ). This applies, furthermore, to large classes of mappings between such spaces, as e.g. nonexpansive, weakly quasi-nonexpansive, Lipschitz-Hölder, uniformly continuous or bounded mappings.

The results in this paper not only allow one to strengthen known existence results in functional analysis by establishing qualitatively new forms of uniform existence as well as by the extractability of explicit quantitative bounds, but also by weakening the assumptions needed. E.g. assumptions of the form ' $f$ has a fixed point' can for large classes of proofs and theorems be replaced by the much weaker assumption ' $f$ has approximate fixed points'. Finally, we will indicate how our results extend to contexts where several spaces $X_{1}, \ldots, X_{n}$ from the aforementioned classes of spaces as well as their products are simultaneously present. We are confident that these results will have many more applications also outside the context of fixed point theory (see [29] and - in particular - [18] for surveys of different topics to which this kind of 'proof mining' approach can be applied).

Let us now sketch the general shape of the results we are going to prove in this paper: we work in an appropriate formal system $\mathcal{A}^{\omega}$ of analysis in which the bulk of classical analysis can be formalized. To this system we add as a kind of atom our abstract structures. E.g. in the case of metric spaces $(X, d)$ or hyperbolic spaces $(X, d, W)$ we add the defining axioms for these structures to our system resulting in systems $\mathcal{A}^{\omega}[X, d]_{-b}$ and $\mathcal{A}^{\omega}[X, d, W]_{-b}$, respectively. Here ' $-b$ ' refers to the fact that in contrast to the systems defined in [25] we do not stipulate that our spaces are bounded. Although we are not able to quantify over the class of all structures of a certain kind we can treat them as parameters and can quantify over the elements of $X$ the functions $f: X \rightarrow X$ and so on which suffices in most cases to represent theorems that hold for these structures. Both in metric spaces as well as in hyperbolic (or CAT(0)) spaces no point $a \in X$ plays any particular role, and so we define a majorizability relation relative to a variable reference point 
$a \in X$. We now describe this relation for some particular cases: An element $x \in X$ is $a$-majorized by a natural number $n \in \mathbb{N}$ (short: $n \gtrsim_{\mathbb{N}}^{a} x$ ) if

$$
n \geq d(x, a) \text {. }
$$

An $a$-majorant of a function $f: X \rightarrow X$ is a function $f^{*}: \mathbb{N} \rightarrow \mathbb{N}$ such that

$$
\forall n \in \mathbb{N}\left(f^{*}(n+1) \geq f^{*}(n) \wedge \forall x \in X\left(n \geq d(x, a) \rightarrow f^{*}(n) \geq d(f(x), a)\right)\right)
$$

and we write $f^{*} \gtrsim_{X \rightarrow X}^{a} f$.

Now suppose that we have a property

$$
A(k, x, f) \equiv \exists m \in \mathbb{N} A_{\exists}(k, x, f, m),
$$

where $A_{\exists}$ is a purely existential formula, $x, f$ range over elements in $X$ and functions $X \rightarrow X$, respectively, and $k \in \mathbb{N}$.

A special form of one of our main results states the following (see Corollaries 4.17 and 4.20): suppose that we can prove in $\mathcal{A}[X, d]_{-b}$ a theorem

$$
\forall k \in \mathbb{N} \forall x \in X \forall f: X \rightarrow X \exists m \in \mathbb{N} A_{\exists}(k, x, f, m) .
$$

Then from that proof one can extract a computable function $\Phi: \mathbb{N} \times \mathbb{N} \times \mathbb{N}^{\mathbb{N}} \rightarrow \mathbb{N}$ such that

$$
\exists m \leq \Phi\left(k, n, f^{*}\right) A_{\exists}(k, x, f, m)
$$

whenever for some $a \in X$

$$
n \gtrsim_{X}^{a} x \text { and } f^{*} \gtrsim_{X \rightarrow X}^{a} f .
$$

Let us now take $a:=x$. Then we can put $n:=0$. For general $f: X \rightarrow X$ an $x$-majorant $f^{*}$ might not exist, but for many classes of functions e.g. Lipschitz continuous functions and - in the case of hyperbolic spaces - uniformly continuous functions, such an $f^{*}$ can easily be constructed. A particularly simple (and important in the context of metric fixed point theory) case is that of nonexpansive functions $f$, i.e.

$$
\forall y, z \in X(d(f(y), f(z)) \leq d(y, z)) .
$$

Then for any $\mathbb{N} \ni b \geq d(x, f(x))$ the function $f_{b}^{*}(m):=m+b$ is an $x$-majorant of $f$. Hence putting $\Psi(k, b):=\Phi\left(k, 0, f_{b}^{*}\right)$ we obtain a computable bound which depends on the $X$-data $x, f$ only via an upper bound $b \geq d(x, f(x))$.

We will give a number of applications to metric fixed point theory. One application (see Application 8.1 below) is concerned with the approximate fixed point property

$$
d\left(x_{n}, f\left(x_{n}\right)\right) \stackrel{n \rightarrow \infty}{\rightarrow} 0
$$

of certain bounded (so-called Krasnoselski-Mann) iterations $\left(x_{n}\right)$ of nonexpansive selfmappings $f: X \rightarrow X$ of hyperbolic spaces starting from an arbitrary point $x_{0}:=$ $x \in X$. Since the sequence $\left(d\left(x_{n}, f\left(x_{n}\right)\right)\right)_{n \in \mathbb{N}}$ can be shown to be nonincreasing, $(*)$ is equivalent to

$$
\forall k, b \in \mathbb{N}\left(\forall n, m \in \mathbb{N}\left(d\left(x_{n}, x_{m}\right) \leq b\right) \rightarrow \exists m \in \mathbb{N}\left(d\left(x_{m}, f\left(x_{m}\right)\right)<2^{-k}\right)\right),
$$

where (representing real numbers as Cauchy sequences of rational numbers with a fixed rate of convergence)

$$
\forall n, m \in \mathbb{N}\left(d\left(x_{n}, x_{m}\right) \leq b\right) \rightarrow \exists m \in \mathbb{N}\left(d\left(x_{m}, f\left(x_{m}\right)\right)<2^{-k}\right)
$$

is logically equivalent to a purely existential formula in the language of $\mathcal{A}^{\omega}[X, d, W]_{-b}$. The assumption $\forall n, m \in \mathbb{N}\left(d\left(x_{n}, x_{m}\right) \leq b\right)$ can be proved to imply 
a bound on $d(x, f(x))$ (in our case $5 b$ suffices) and so in total one can extract a computable $\Psi(k, b)^{1}$ such that (using that the sequence $\left(d\left(x_{n}, f\left(x_{n}\right)\right)\right)_{n \in \mathbb{N}}$ is nonincreasing) the following holds in all hyperbolic spaces $(X, d, W)$, all points $x \in X$ and all nonexpansive functions $f: X \rightarrow X$ :

$$
\forall k, b \in \mathbb{N}\left(\forall n, m \in \mathbb{N}\left(d\left(x_{n}, x_{m}\right) \leq b\right) \rightarrow \forall m \geq \Psi(k, b)\left(d\left(x_{m}, f\left(x_{m}\right)\right)<2^{-k}\right)\right) .
$$

The most important aspect of this result is that the rate of convergence is - except for the bound $b$ on the sequence $\left(x_{n}\right)$ - totally independent from the starting point $x$, the function $f$ and the space $X$. E.g. if $X$ is a bounded space, then we get total independence from $x, f$.

The bound $\Psi$ extractable from the proof not only is computable but of certain limited computational complexity (depending on the principles used in the given proof). The proof of the metatheorem provides an algorithm for actually extracting $\Psi$ from a given proof, and in many cases this has been carried out (see e.g. [21, 22, $23,24,26,31,32]$ and the discussion in section 8 below).

\section{Definitions}

The classes of general spaces we are considering are metric spaces, hyperbolic spaces (including CAT(0)-spaces), as well as normed spaces. By a hyperbolic space we understand the following:

Definition 2.1. $(X, d, W)$ is called a hyperbolic space if $(X, d)$ is a metric space and $W: X \times X \times[0,1] \rightarrow X$ a function satisfying
(i) $\forall x, y, z \in X \forall \lambda \in[0,1](d(z, W(x, y, \lambda)) \leq(1-\lambda) d(z, x)+\lambda d(z, y))$,
(ii) $\forall x, y \in X \forall \lambda_{1}, \lambda_{2} \in[0,1]\left(d\left(W\left(x, y, \lambda_{1}\right), W\left(x, y, \lambda_{2}\right)\right)=\left|\lambda_{1}-\lambda_{2}\right| \cdot d(x, y)\right)$,
(iii) $\forall x, y \in X \forall \lambda \in[0,1](W(x, y, \lambda)=W(y, x, 1-\lambda))$,
(iv) $\left\{\begin{array}{l}\forall x, y, z, w \in X, \lambda \in[0,1] \\ (d(W(x, z, \lambda), W(y, w, \lambda)) \leq(1-\lambda) d(x, y)+\lambda d(z, w)) .\end{array}\right.$

Remark 2.2. As discussed in detail in [25], we obtain Takahashi's [41] 'convex metric spaces' if the axioms (ii)-(iv) are dropped, and a notion which is equivalent to the concept of 'space of hyperbolic type' from [11] if we drop only (iv). As observed in $[37,2]$ and [38], several arguments in metric fixed point theory require a bit more linear structure which gave rise to a notion of 'hyperbolic space'" in $[14,37]$ which adds axiom (iv) (for $\lambda:=\frac{1}{2}$ ) and the requirement that any two points are not only connected by a metric segment but by a metric line. As a consequence of this (just as in the case of normed spaces) nontrivial hyperbolic spaces in the sense of $[14,37]$ always are unbounded and convex subsets of a hyperbolic space in general are no longer hyperbolic spaces themselves. The existence of metric lines allows one to derive the general axiom (iv) from the special case of $\lambda=\frac{1}{2}$. It turns out that if we state (iv) directly for general $\lambda$ as above, then the proofs in metric fixed point theory we are interested in all go through for our more liberal notion of 'hyperbolic space' which not only has a simpler logical structure but also includes all convex subsets of hyperbolic (and in particular normed) spaces as well as all CAT(0)-spaces, whereas the more restricted notion used in $[14,37,38]$ only covers

\footnotetext{
${ }^{1}$ More precisely, $\Psi$ additionally depends on two parameters having to do with the sequence of scalars used to define the iteration.

${ }^{2}$ Unfortunately, Kirk calls this notion in [14] 'space of hyperbolic type' although it differs from the definition of the latter in [11].
} 
CAT(0)-spaces having the geodesic line extension property (see [3] and below for details on $\operatorname{CAT}(0)$-spaces).

As carried out in detail in [25] we formalize our classes of spaces on top of a formal system $\mathcal{A}^{\omega}$ of classical analysis which is based on a language of functionals of finite type.

Definition 2.3. The set $\mathbf{T}$ of all finite types is defined inductively over the ground type 0 by the clauses

$$
\text { (i) } 0 \in \mathbf{T} \text {, (ii) } \rho, \tau \in \mathbf{T} \Rightarrow(\rho \rightarrow \tau) \in \mathbf{T} \text {. }
$$

The type 0 represents natural numbers and so we sometimes use ' $\mathbb{N}$ ' instead of ' 0 '. Objects of type $\rho \rightarrow \tau$ represent operations that map objects of type $\rho$ to objects of type $\tau$.

A system $\mathcal{A}^{\omega}$ for analysis (which is based on the axioms of countable and dependent choice which, in particular, yield full comprehension for numbers) is defined as in [25]. Higher type equality is not a primitive predicate but is defined extensionally. Instead of the full axiom of extensionality in all types, the system $\mathcal{A}^{\omega}$ only has a quantifier-free rule of extensionality. ${ }^{3}$

Before we can describe the extensions $\mathcal{A}^{\omega}[X, d], \mathcal{A}^{\omega}[X, d, W]$, etc. of $\mathcal{A}^{\omega}$ by an abstract metric space $(X, d)$ or hyperbolic space $(X, d, W)$ we briefly have to recall the representation of real numbers in the formal system $\mathcal{A}^{\omega}$ :

In our formal systems based on $\mathcal{A}^{\omega}$, real numbers are represented by Cauchy sequences $\left(a_{n}\right)_{n \in \mathbb{N}}$ of rational numbers with Cauchy modulus $2^{-n}$, i.e.

$$
\forall m, n\left(m, n \geq k \rightarrow\left|a_{m}-a_{n}\right|<2^{-k}\right) .
$$

Rational numbers are represented as pairs $(n, m)$ of natural numbers coded into a single natural number $j(n, m)$, where $j$ is the Cantor pairing function. If $n$ is even $j(n, m)$ represents the rational number $\frac{n / 2}{m+1}$, and if $n$ is odd $j(n, m)$ represents the negative number $-\frac{(n+1) / 2}{m+1}$. Thus every natural number can be conceived of as the code of a unique rational number. An equality relation $=_{\mathbb{Q}}$ on the representatives of the rational numbers, as well as the usual operators $+_{\mathbb{Q}},-_{\mathbb{Q}}, \cdot_{\mathbb{Q}}$, etc. and the predicates $<_{\mathbb{Q}}, \leq_{\mathbb{Q}}$, are defined (primitive recursively) in the obvious way. Thus natural and rational numbers are represented by objects of type 0 and sequences of rational numbers by objects of type 1 , i.e. by functions of type $0 \rightarrow 0$.

Real numbers are represented by functions $f: \mathbb{N} \rightarrow \mathbb{N}$ (i.e. of type 1 ) s.t.

$$
\forall n\left(|f(n)-\mathbb{Q} f(n+1)|<\mathbb{Q} 2^{-n-1}\right) .
$$

To ensure that each function $f: \mathbb{N} \rightarrow \mathbb{N}$ represents a real number we use the following construction:

$$
\widehat{f}(n):=\left\{\begin{array}{l}
f(n) \text { if } \forall k<n\left(|f(k)-\mathbb{Q} f(k+1)|_{\mathbb{Q}}<\mathbb{Q} 2^{-k-1}\right), \\
f(k) \text { for } \min k<n \text { with }|f(k)-\mathbb{Q} f(k+1)|_{\mathbb{Q}} \geq_{\mathbb{Q}} 2^{-k-1} \text { otherwise. }
\end{array}\right.
$$

For better readability we usually write e.g. $2^{-n}$ instead of its (canonical) code $\left\langle 2^{-n}\right\rangle:=j\left(2,2^{n}-1\right)$.

For every $f: \mathbb{N} \rightarrow \mathbb{N}$ the construction $\widehat{f}$, which can be carried out in $\mathcal{A}^{\omega}$, satisfies $(*)$, and if $f$ already satisfies $(*)$, then $\forall n\left(f(n)=_{0} \widehat{f}(n)\right)$. Thus every $f$ codes a unique real number, namely the one given by the Cauchy sequence coded by $\widehat{f}$.

\footnotetext{
${ }^{3}$ See $[25]$ for an extensive discussion of this crucial point.
} 
The construction $f \mapsto \widehat{f}$ enables us to reduce quantifiers ranging over $\mathbb{R}$ to $\forall f^{1}$, resp. $\exists f^{1}$, without introducing additional quantifiers. For natural numbers $b \in \mathbb{N}$ we have the embedding $(b)_{\mathbb{R}}$ via the construction $(b)_{\mathbb{R}}={ }_{1} \lambda n \cdot j(2 b, 0)$.

The equivalence relation $=_{\mathbb{R}}$ and the relations $\leq_{\mathbb{R}}$ and $<_{\mathbb{R}}$ on (representatives of) real numbers are defined notions. The relations $=_{\mathbb{R}}$ and $\leq_{\mathbb{R}}$ are given by $\Pi_{1}^{0}$ predicates while $<_{\mathbb{R}}$ is given by a $\Sigma_{1}^{0}$-predicate:

$$
\begin{aligned}
& f_{1}=_{\mathbb{R}} f_{2}: \equiv \forall n\left(\left|\widehat{f}_{1}(n+1)-\mathbb{Q} \widehat{f}_{2}(n+1)\right|<_{\mathbb{Q}} 2^{-n}\right), \\
& f_{1}<_{\mathbb{R}} f_{2}: \equiv \exists n\left(\widehat{f}_{2}(n+1)-\widehat{\mathbb{f}}_{1}(n+1) \geq_{\mathbb{Q}} 2^{-n}\right), \\
& f_{1} \leq_{\mathbb{R}} f_{2}: \equiv \neg\left(f_{2}<_{\mathbb{R}} f_{1}\right) .
\end{aligned}
$$

The operators $+_{\mathbb{R}},-\mathbb{R}, \cdot \mathbb{R}$, etc. on representatives of real numbers can be defined by simple primitive recursive functionals. For further details see [25].

For the interval $[0,1]$, which plays an important role in the formal treatment of hyperbolic spaces, we use a special representation by number theoretic functions $\mathbb{N} \rightarrow \mathbb{N}$ (which are bounded by a fixed function $M$ ):

\section{Definition 2.4.}

$$
\tilde{x}(n):=j\left(2 k_{0}, 2^{n+2}-1\right), \text { where } k_{0}=\max k \leq 2^{n+2}\left[\frac{k}{2^{n+2}} \leq_{\mathbb{Q}} \widehat{x}(n+2)\right]
$$

(if $k$ does not exist we take $k_{0}:=0$; recall that $j\left(2 k_{0}, 2^{n+2}-1\right)$ encodes the rational number $\left.k_{0} / 2^{n+2}\right)$.

One easily verifies the following:

Lemma 2.5. Provably in $\mathcal{A}^{\omega}$, for all $x^{1}$ :

(1) $0_{\mathbb{R}} \leq_{\mathbb{R}} x \leq_{\mathbb{R}} 1_{\mathbb{R}} \rightarrow \tilde{x}=\mathbb{R} x$,

(2) $0_{\mathbb{R}} \leq_{\mathbb{R}} \tilde{x} \leq_{\mathbb{R}} 1_{\mathbb{R}}$

(3) $\tilde{x} \leq_{1} M:=\lambda n \cdot j\left(2^{n+3}, 2^{n+2}-1\right)$,

(4) $x>_{\mathbb{R}} 1_{\mathbb{R}} \rightarrow \tilde{x}=\mathbb{R} 1_{\mathbb{R}}, x<_{\mathbb{R}} 0_{\mathbb{R}} \rightarrow \tilde{x}=_{\mathbb{R}} 0_{\mathbb{R}}$

The theories of classical analysis extended with metric or normed linear spaces and their variants are defined almost as in [25]. The crucial difference is that while in [25] only bounded metric spaces $(X, d)$ and bounded convex subsets $C$ of normed linear spaces $(X,\|\cdot\|)$ are considered, we now permit unbounded metric spaces $(X, d)$ and unbounded convex subsets $C$. In [25], the boundedness is expressed by an axiom stating explicitly that $(X, d)$, resp. the convex subsets $C$, are bounded by $b$. In our unbounded variants we omit this axiom. To distinguish the unbounded theories from the $b$-bounded theories $\mathcal{A}^{\omega}[X, d]$ and $\mathcal{A}^{\omega}[X,\|\cdot\|, C]$ defined in [25], we will denote the former by $\mathcal{A}^{\omega}[X, d]_{-b}$ and $\mathcal{A}^{\omega}[X,\|\cdot\|, C]_{-b}$. We also consider theories $\mathcal{A}^{\omega}[X, d, W]_{-b}$ capturing hyperbolic spaces and $\mathcal{A}^{\omega}[X, d, W, \operatorname{CAT}(0)]_{-b}$ capturing CAT(0)-spaces. More precisely, the theories $\mathcal{A}^{\omega}[X, d]_{-b}, \mathcal{A}^{\omega}[X, d, W]_{-b}$ and $\mathcal{A}^{\omega}[X, d, W, \operatorname{CAT}(0)]_{-b}$ result by

(i) extending $\mathcal{A}^{\omega}$ to the set $\mathbf{T}^{X}$ of all finite types over the two ground types 0 and $X$, i.e.

$$
\text { (i) } 0, X \in \mathbf{T}^{X} \text {, (ii) } \rho, \tau \in \mathbf{T}^{X} \Rightarrow(\rho \rightarrow \tau) \in \mathbf{T}^{X}
$$

(in particular, the constants $\Pi_{\rho, \tau}, \Sigma_{\delta, \rho, \tau}, \underline{R}_{\rho}$ for $\lambda$-abstraction and simultaneous primitive recursion (in the extended sense of Gödel [10]) and their defining axioms and the schemes IA (induction), QF-AC (quantifier-free 
choice in all types), DC (dependent countable choice) ${ }^{4}$ and the weak extensionality rule QF-ER are now taken over the extended language),

(ii) adding a constant $0_{X}$ of type $X$,

(iii) adding a new constant $d_{X}$ of type $X \rightarrow X \rightarrow 1$ (representing the metric) together with the axioms

(1) $\forall x^{X}\left(d_{X}(x, x)=_{\mathbb{R}} 0_{\mathbb{R}}\right)$,

(2) $\forall x^{X}, y^{X}\left(d_{X}(x, y)=_{\mathbb{R}} d_{X}(y, x)\right)$,

(3) $\forall x^{X}, y^{X}, z^{X}\left(d_{X}(x, z) \leq_{\mathbb{R}} d_{X}(x, y)+_{\mathbb{R}} d_{X}(y, z)\right)$.

In these axioms we refer to the representation of real numbers (including the definition of $=_{\mathbb{R}}, \leq_{\mathbb{R}}$ ) as sketched above.

Equality $=_{0}$ at type 0 is the only primitive equality predicate. $x^{X}={ }_{X} y^{X}$ is defined as $d_{X}(x, y)={ }_{\mathbb{R}} 0_{\mathbb{R}}$. Equality for complex types is defined as before as extensional equality using $=_{0}$ and $=_{X}$ for the base cases.

$\mathcal{A}^{\omega}[X, d, W]_{-b}$ results from $\mathcal{A}^{\omega}[X, d]_{-b}$ by adding a new constant $W_{X}$ of type $X \rightarrow X \rightarrow 1 \rightarrow X$ together with the axioms (where $\tilde{\lambda}$ is defined as above)

(4) $\forall x^{X}, y^{X}, z^{X} \forall \lambda^{1}\left(d_{X}\left(z, W_{X}(x, y, \lambda)\right) \leq_{\mathbb{R}}\left(1_{\mathbb{R}}-\mathbb{R}\right) d_{X}(z, x)+_{\mathbb{R}} \tilde{\lambda} d_{X}(z, y)\right)$,

(5) $\forall x^{X}, y^{X} \forall \lambda_{1}^{1}, \lambda_{2}^{1}\left(d_{X}\left(W_{X}\left(x, y, \lambda_{1}\right), W_{X}\left(x, y, \lambda_{2}\right)\right)={ }_{\mathbb{R}} \mid \tilde{\lambda}_{1}-\tilde{R}_{\mathbb{R}} \tilde{\lambda}_{\mathbb{R}} \cdot \mathbb{R} d_{X}(x, y)\right)$,

(6) $\forall x^{X}, y^{X} \forall \lambda^{1}\left(W_{X}(x, y, \lambda)={ }_{X} W_{X}\left(y, x,\left(1_{\mathbb{R}}-\mathbb{R} \lambda\right)\right)\right)$,

(7) $\left\{\begin{array}{l}\forall x^{X}, y^{X}, z^{X}, w^{X}, \lambda^{1} \\ \left(d_{X}\left(W_{X}(x, z, \lambda), W_{X}\right.\right.\end{array}\right.$

$\mathcal{A}^{\omega}[X, d, W, \operatorname{CAT}(0)]_{-b}$ results from $\mathcal{A}^{\omega}[X, d, W]_{-b}$ by adding as a further axiom the formalized form of the Bruhat-Tits or $\mathbf{C N}^{-}$-inequality [4], i.e.

$\forall x^{X}, y_{1}^{X}, y_{2}^{X}\left(d_{X}\left(x, W_{X}\left(y_{1}, y_{2}, \frac{1}{2}\right)\right)^{2} \leq_{\mathbb{R}} \frac{1}{2} d_{X}\left(x, y_{1}\right)^{2}+\mathbb{R} \frac{1}{2} d_{X}\left(x, y_{2}\right)^{2}-\mathbb{R} \frac{1}{4} d_{X}\left(y_{1}, y_{2}\right)^{2}\right)$.

Remark 2.6. (1) The additional axioms of $\mathcal{A}^{\omega}[X, d]_{-b}$ express (modulo our representation of $\mathbb{R}$ sketched above) that $d_{X}$ represents a pseudo-metric $d$ (on the universe the type- $X$ variables are ranging over). ${ }^{5}$ Hence $d_{X}$ represents a metric on the set of equivalence classes generated by $=_{X}$. We do not form these equivalence classes explicitly but talk instead only about representatives $x^{X}, y^{X}$. However, it is important to stress that a functional $f^{X \rightarrow X}$ represents a function $X \rightarrow X$ only if it respects this equivalence relation, i.e.

$$
\forall x^{X}, y^{X}\left(x=_{X} y \rightarrow f(x)={ }_{X} f(y)\right) .
$$

Due to our weak (quantifier-free) rule of extensionality we in general only can infer from a proof of $s={ }_{X} t$ that $f(s)={ }_{X} f(t)$. The restriction on the availability of extensionality is crucial for our results to hold (see the discussion in [25]). However, the full extensionality of the constants $d_{X}, W_{X}$ as well as the constants for normed linear spaces can all be proved from their defining axioms. Likewise, for most (but not all) of the classes of functions which we will consider below (notably the nonexpansive functions) the full extensionality will follow from their defining properties.

\footnotetext{
${ }^{4}$ We allow in both QF-AC and DC tuples of variables.

${ }^{5}$ Note that $(1)-(3)$ imply that $\forall x^{X}, y^{X}\left(d_{X}(x, y) \geq \geq_{\mathbb{R}} 0_{\mathbb{R}}\right)$.
} 
(2) Our axiomatization of $W_{X}$ given by the axioms (4)-(7) differs slightly from the one given in [25]. Our present axiomatization is equivalent to the extension of the one given in [25] by the additional axiom

$$
W_{X}(x, y, \lambda)=_{X} W_{X}(x, y, \tilde{\lambda})
$$

using the property

$$
\widetilde{1-\mathbb{R} \lambda}=\mathbb{R} 1-\mathbb{R} \tilde{\lambda}
$$

of our operation $\lambda \mapsto \tilde{\lambda}$ (which follows using Lemma 2.5 (4)). This additional axiom is trivially satisfied by the interpretation of $W_{X}$ in the model $\mathcal{S}^{\omega, X}$ from [25] so that it can be added without causing problems. The benefit of this is that then the axioms on $W_{X}$ can be stated in the simple form given above compared to the more complicated formulation in [25]. The intuitive interpretation of $W_{X}$ in a hyperbolic space $(X, d, W)$ is that for $x, y \in X$ we interpret $W_{X}(x, y, \lambda)$ by $W\left(x, y, r_{\tilde{\lambda}}\right)$ where $r_{\tilde{\lambda}}$ is the unique real number in $[0,1]$ that is represented by $\tilde{\lambda}$.

(3) Our additional axiom $\mathbf{C N}^{-}$used in defining $\mathcal{A}^{\omega}[X, d, W, \mathrm{CAT}(0)]$ differs from the one used in [25] (under the name $\mathbf{C N}^{*}$ ) but is in fact equivalent to the latter: $\mathbf{C N}^{-}$is (over the $W$-axioms (i)-(iv)) equivalent to the more usual formulation $\mathbf{C N}$ of the Bruhat-Tits inequality [4] which states that every midpoint of $y_{1}, y_{2}$ satisfies the inequality stated for $W\left(y_{1}, y_{2}, \frac{1}{2}\right)$. The latter point provably (in $\mathcal{A}^{\omega}[X, d, W]$ ) is a midpoint so that $\mathbf{C N}$ implies $\mathbf{C N}^{-}$. From $\mathbf{C N}^{-}$it easily follows not only that every midpoint of $y_{1}, y_{2}$ has to coincide with $W\left(y_{1}, y_{2}, \frac{1}{2}\right)$ (so that $\mathbf{C N}$ follows) but even the quantitative version $\mathbf{C N}^{*}$ of $\mathbf{C N}$. In [25], $\mathbf{C N}^{*}$ was used as an axiom as it is (in contrast to $\mathbf{C N}$ ) purely universal which is crucially used in the proofs. However, $\mathbf{C N}^{-}$is purely universal too and equivalent to $\mathbf{C N}^{*}$. Since it is easier to state, we use this formulation here.

As for $\mathcal{A}^{\omega}[X,\|\cdot\|, C]_{-b}$, the corresponding theories for uniformly convex spaces, $\mathcal{A}^{\omega}[X,\|\cdot\|, C, \eta]_{-b}$, and real inner product spaces, $\mathcal{A}^{\omega}[X,\langle\cdot, \cdot\rangle, C]_{-b}$, are defined as in [25] except that the axioms stating the boundedness of $C$ is dropped. ${ }^{6}$

Finally, various moduli naturally occurring in analysis, such as e.g. a modulus of uniform continuity or a modulus of uniform convexity, are also represented by number theoretic functions $\mathbb{N} \rightarrow \mathbb{N}$, i.e. objects of type 1 . Thus e.g. the statement $f: X \rightarrow X$ is uniformly continuous with modulus $\omega: \mathbb{R}_{+}^{*} \rightarrow \mathbb{R}_{+}^{*}$ :

$$
\forall x, y \in X \forall \varepsilon>0(d(x, y) \leq \omega(\varepsilon) \rightarrow d(f(x), f(y)) \leq \varepsilon)
$$

is translated into

$$
\forall x, y \in X \forall k \in \mathbb{N}\left(d(x, y)<2^{-\omega(k)} \rightarrow d(f(x), f(y)) \leq 2^{-k}\right)
$$

where $\omega: \mathbb{N} \rightarrow \mathbb{N}$ and the translated statement is purely universal.

\section{A generalized APproach to majorization}

In [25] the strong majorization relation, first introduced by Bezem [1] for the finite types $\mathbf{T}$ over $\mathbb{N}$, is extended to the types $\mathbf{T}^{X}$ with the new ground type $X$ for metric spaces $(X, d)$ and normed linear spaces $(X,\|\cdot\|)$. Furthermore, a mapping $\widehat{\rho}$ between types $\rho \in \mathbf{T}^{X}$ and $\widehat{\rho} \in \mathbf{T}$, and a relation $\sim_{\rho}$ between functionals of type $\rho \in \mathbf{T}^{X}$ and $\widehat{\rho} \in \mathbf{T}$ are defined inductively. By relating the constants of the theories

\footnotetext{
${ }^{6}$ Correction to $[25]$ : add the axiom $\left\|1_{X}\right\|={ }_{\mathbb{R}}(1)_{\mathbb{R}}$ to $\mathcal{A}^{\omega}[X,\|\cdot\|, C]$.
} 
$\mathcal{A}^{\omega}[X, d]$ and $\mathcal{A}^{\omega}[X,\|\cdot\|]$ (and their variants) to suitable functionals in $\mathcal{A}^{\omega}$ via the relation $\sim_{\rho}$, one can, combined with majorization in the types $\mathbf{T}^{X}$, systematically eliminate the dependency on the type $X$ in the extracted terms and obtain bounds independent of parameters ranging over bounded metric spaces, resp. bounded convex subsets of normed linear spaces.

In this section we present a generalized approach to extending the strong majorization relation to the types $\mathbf{T}^{X}$. The (strong) majorization relation was defined by Howard and Bezem:

Definition 3.1 (Howard-Bezem, $[12,1]$ ). The strong majorization relation s-maj over the finite types $\mathbf{T}$ is defined as follows:

- $x^{*}{ }^{\mathrm{s}-\mathrm{maj}_{0}} x: \equiv x^{*} \geq_{\mathbb{N}} x$, where $\geq_{\mathbb{N}}$ is the usual (primitive recursive) order on $\mathbb{N}$

- $x^{*}{ }^{\mathrm{s}-\mathrm{maj}_{\rho \rightarrow \tau}} x: \equiv \forall y^{*}, y\left(y^{*} \mathrm{~s}_{\rho}-\mathrm{maj}_{\rho} y \rightarrow x^{*} y^{*}{ }_{\mathrm{s}-\mathrm{maj}_{\tau}} x^{*} y, x y\right)$.

In [25], two different approaches are employed for metric and normed linear spaces, respectively, to extend the majorization relation to the new type $X$. For metric spaces only the restricted case of $b$-bounded spaces is treated, where $b$ is an integer upper bound on the metric of the space. For bounded metric spaces the relation s-maj is extended to the types $\mathbf{T}^{X}$ by defining:

$$
x^{*}{ }^{\mathrm{s}-\mathrm{maj}_{X}} x: \equiv(0=0) \text {, i.e. always true. }
$$

Usually extending majorization to a new type $X$ imposes a kind of order on the elements of $X$ which the majorization of the constants $0_{X}, d_{X}$ and $W_{X}$ must respect. Since here the metric $d_{X}$ can be bounded independently of the elements $x, y \in X$ to which it is applied, namely by $\lambda x^{X}, y^{X} .(b)_{\mathbb{R}}$, and since the function $W_{X}$ merely produces new elements of $X$, in [25] the majorization relation on $X$ could be defined to be always true (corresponding to a trivial order on $X$ ).

For normed linear spaces this approach does not work, as nontrivial normed linear spaces always are unbounded. Instead in [25] the extension of the majorization relation to the new type $X$ for normed linear spaces $(X,\|\cdot\|)$ is defined via the norm:

$$
x^{*}{ }^{\mathrm{s}-\mathrm{maj}_{X}} x: \equiv\left\|x^{*}\right\|_{X} \geq_{\mathbb{R}}\|x\|_{X} .
$$

The majorization of extracted terms in [25] then consists of three steps: First one majorizes the extracted terms in $\mathbf{T}^{X}$ - these majorants may still depend on some of the constants of $\mathcal{A}^{\omega}[X,\|\cdot\|]$. Next one eliminates the dependency on $X$ using the relation $\sim_{\rho}$ and an ineffective operator $(\cdot)_{\circ}$ (to be defined below). Finally, the resulting terms are majorized once more in the types $\mathbf{T}$ to eliminate uses of the ineffective $(\cdot)_{\circ}$-operator.

As mentioned above, using these techniques it is possible to derive the independence of extracted bounds from parameters ranging over bounded metric spaces, resp. norm-bounded convex subsets $C$ of normed linear spaces. The generalized approach to majorization we describe in this section aims to treat the more general cases of unbounded metric and hyperbolic spaces and normed linear spaces with unbounded convex subsets $C$, i.e. the theories $\mathcal{A}^{\omega}[X, d]_{-b}, \mathcal{A}^{\omega}[X, d, W]_{-b}$ and $\mathcal{A}^{\omega}[X,\|\cdot\|, C]_{-b}$, and to derive similar uniformities under certain local boundedness conditions to be discussed in detail later. This generalized approach is based upon the first two steps of the previous treatment of normed linear spaces: (strong) majorization in the types $\mathbf{T}^{X}$ and the relation $\sim_{\rho}$. In [25], by the mapping $\widehat{\cdot}$, the 
type $X$ with $(X, d)$ a metric space, was mapped to the type 0 , while the type $X$ with $(X,\|\cdot\|)$ a normed linear space was mapped to the type 1 . In this paper, we will map the type $X$ to 0 in both cases:

Definition 3.2. For $\rho \in \mathbf{T}^{X}$ we define $\widehat{\rho} \in \mathbf{T}$ inductively as follows:

$$
\widehat{0}:=0, \widehat{X}:=0,(\widehat{\rho \rightarrow \tau}):=(\widehat{\rho} \rightarrow \widehat{\tau}),
$$

i.e. $\widehat{\rho}$ is the result of replacing all occurrences of the type $X$ in $\rho$ by the type 0 .

The generalized approach to majorization will again involve the ()o-operator, but restricted to cases where the circle operator is effectively computable. Hence, the second application of strong majorization, used in the previous treatment of normed linear spaces to get rid of ineffective instances of the ()o-operator, is no longer necessary.

Combining Bezem's notion of strong majorization s-maj and the idea of the relation $\sim \rho$ we define a family of (majorization) relations $\gtrsim_{\rho}^{a}$ between objects of type $\rho \in \mathbf{T}^{X}$ and their majorants of type $\widehat{\rho} \in \mathbf{T}$. The relation is parametrized by an element $a \in X$, where $X$ is the underlying metric or normed linear space and $a \in X$ serves as a reference point for comparing and majorizing elements of $X$. In $\mathcal{L}\left(\mathcal{A}^{\omega}[X, d]_{-b}\right)$, resp. $\mathcal{L}\left(\mathcal{A}^{\omega}[X,\|\cdot\|]\right)$, this is syntactically expressed as follows:

Definition 3.3. We define a ternary relation $\gtrsim_{\rho}^{a}$ between objects $x, y$ and $a$ of type $\widehat{\rho}, \rho$ and $X$, respectively, by induction on $\rho$ as follows:

- $x^{0} \gtrsim_{0}^{a} y^{0}: \equiv x \geq_{\mathbb{N}} y$,

- $x^{0} \gtrsim_{X}^{a} y^{X}: \equiv(x)_{\mathbb{R}} \geq_{\mathbb{R}} d_{X}(y, a)$,

- $x \gtrsim_{\rho \rightarrow \tau}^{a} y: \equiv \forall z^{\prime}, z\left(z^{\prime} \gtrsim_{\rho}^{a} z \rightarrow x z^{\prime} \gtrsim_{\tau}^{a} y z\right) \wedge \forall z^{\prime}, z\left(z^{\prime} \gtrsim_{\widehat{\rho}}^{a} z \rightarrow x z^{\prime} \gtrsim_{\widehat{\tau}}^{a} x z\right)$.

For normed linear spaces we choose $a=0_{X},{ }^{7}$ so that $d_{X}(x, a)=\mathbb{R}\|x\|_{X}$.

As $\gtrsim^{a}$ is a relation between objects of different types, the definition of $\gtrsim_{\rho \rightarrow \tau}^{a}$ is slightly more complicated than the corresponding definition of s-maj $\mathrm{j}_{\rho \rightarrow \tau}$. The first part of the clause ensures that $x$ is a "majorant" for $y$, and the second part ensures that a majorant $x$ also majorizes itself. Since majorants are of type $\widehat{\rho} \in \mathbf{T}$ (where $\gtrsim_{\widehat{\rho}}^{a}$ coincides with $\mathrm{s}-\mathrm{maj}_{\hat{\rho}}$ ), this corresponds to requiring that for all majorants $x$ s-maj $x$. Hence the definition of $\gtrsim_{\rho \rightarrow \tau}^{a}$ could equivalently be rewritten as:

$$
x \gtrsim_{\rho \rightarrow \tau}^{a} y: \equiv \forall z^{\prime}, z\left(z^{\prime} \gtrsim_{\rho}^{a} z \rightarrow x z^{\prime} \gtrsim_{\tau}^{a} y z\right) \wedge x \operatorname{s}^{-m_{a j} \rightarrow \widehat{\tau}}{ }_{\widehat{\rho}} x .
$$

Remark 3.4. Restricted to the types $\mathbf{T}$ the relation $\gtrsim^{a}$ is identical with the HowardBezem notion of strong majorizability s-maj, and hence for $\rho \in \mathbf{T}$ we may freely write s-maj $j_{\rho}$ instead of $\gtrsim_{\rho}^{a}$, as here the parameter $a \in X$ is irrelevant. Without the requirement that "majorants" must be strongly self-majorizing, $\gtrsim^{a}$ restricted to $\mathbf{T}$ is identical with Howard's notion of majorizability maj.

In the following, we call majorization in the sense of the relation $\gtrsim^{a}$ (strong) " $a$-majorization", i.e. if $t_{1} \gtrsim^{a} t_{2}$ for terms $t_{1}, t_{2}$ we say that $t_{1} a$-majorizes $t_{2}$ and we call $t_{1}$ an $a$-majorant. If neither term $t_{i}$ depends on $a$ we say that $t_{1}$ uniformly $a$-majorizes $t_{2}$. We will in general aim at uniform majorants so that we can choose $a$ appropriately (without having an effect on the majorants of the constants of our theories) to obtain bounds with the intended uniformity features.

\footnotetext{
${ }^{7}$ While it will turn out to be independent of the choice of $a$ whether a given functional is $a$-majorizable or not, the choice of $a$ is crucial to obtain "nice" majorants. See Section 9 for a detailed discussion.
} 
For the normed case we also need a pointwise $\geq_{\rho}$ relation between functionals of type $\rho$ :

Definition 3.5. $\geq_{\rho}$ is a binary relation between functionals of type $\rho \in \mathbf{T}^{X}$ and which is defined by induction on $\rho$ as follows:

- $x^{0} \geq_{0} y^{0}: \equiv x \geq_{\mathbb{N}} y$,

- $x^{X} \geq_{X} y^{X}: \equiv\|x\|_{X} \geq_{\mathbb{R}}\|y\|_{X}$,

- $x \geq_{\rho \rightarrow \tau} y: \equiv \forall z^{\rho}\left(x z \geq_{\tau} y z\right)$.

Lemma 3.6. For all $x^{*}, x, y$ of type $\hat{\rho}, \rho, \rho$, resp., the following holds (provably in $\left.\mathcal{A}^{\omega}[X,\|\cdot\|]\right)$ :

$$
x^{*} \gtrsim_{\rho}^{0_{X}} x \wedge x \geq_{\rho} y \rightarrow x^{*} \gtrsim_{\rho}^{0_{X}} y .
$$

Proof. Easy induction on $\rho \in \mathbf{T}^{X}$.

\section{Metatheorems For Metric AND hyperbolic SPACES}

Before we state the new metatheorems, we recall and add the following definitions:

Definition 4.1 ([25]). We say that a type $\rho \in \mathbf{T}^{X}$ has degree

- 1 if $\rho=0 \rightarrow \ldots \rightarrow 0$ (including $\rho=0$ ),

- 2 if $\rho=\tau_{1} \rightarrow \ldots \rightarrow \tau_{k} \rightarrow 0$ (including $\rho=0$ ) with $\tau_{1}, \ldots, \tau_{k}$ of degree 1 ,

- $(0, X)$ if $\rho=0 \rightarrow \ldots \rightarrow 0 \rightarrow X$ (including $\rho=X$ ),

- $(1, X)$ if it has the form $\tau_{1} \rightarrow \ldots \rightarrow \tau_{k} \rightarrow X$ (including $\rho=X$ ), where $\tau_{i}$ has degree 1 or $(0, X)$.

Definition 4.2. We say that a type $\rho \in \mathbf{T}^{X}$ has degree $\hat{1}$ if $\widehat{\rho}$ has degree 1 . Amongst others, the type degree $\hat{1}$ covers (writing $\mathbb{N}$ instead of 0 ) types $\mathbb{N}, X, \mathbb{N} \rightarrow$ $\mathbb{N}, \mathbb{N} \rightarrow X, X \rightarrow \mathbb{N}$ and $X \rightarrow X$.

Definition 4.3. A formula $F$ is called a $\forall$-formula (resp. $\exists$-formula) if it has the form $F \equiv \forall \underline{a} \underline{\underline{\sigma}} F_{q f}(\underline{a})$ (resp. $F \equiv \exists \underline{a} \underline{\underline{\sigma}} F_{q f}(\underline{a})$ ) where $F_{q f}$ does not contain any quantifiers and the types in $\underline{\sigma}$ are of degree $\hat{1}$ or $(1, X)$.

The ()o-operator is defined as follows:

Definition $4.4([25])$. For $x \in[0, \infty)$ define $(x)_{\circ} \in \mathbb{N}^{\mathbb{N}}$ by

$$
(x)_{\circ}(n):=j\left(2 k_{0}, 2^{n+1}-1\right) \text {, }
$$

where

$$
k_{0}:=\max k\left[\frac{k}{2^{n+1}} \leq x\right]
$$

Remark 4.5. ()。 is a 'semantic' operator defined on the real numbers themselves (rather than representatives of real numbers). However, it has a counterpart 0 : $\mathbb{N}^{\mathbb{N}} \rightarrow \mathbb{N}^{\mathbb{N}}$ defined as $\left(f^{1}\right)_{\circ}=(|r|)_{\circ}$, where $r$ is the real number represented by $f$, i.e. $\left(f^{1}\right)_{\circ} \in \mathbb{N}^{\mathbb{N}}$ is defined by

$$
(f)_{\circ}(n):=j\left(2 k_{0}, 2^{n+1}-1\right)
$$

where

$$
k_{0}:=\max k\left[\frac{k}{2^{n+1}} \leq_{\mathbb{R}}|f|_{\mathbb{R}}\right] .
$$

In contrast to $\tilde{f}^{1}$ defined before, this functional of type $1 \rightarrow 1$ is not computable, but in our bounds it will only be used in the form $\lambda n^{0} \cdot\left((n)_{\mathbb{R}}\right)$ 。 which is elementary 
computable: $\left((n)_{\mathbb{R}}\right)_{\circ}(k)=j\left(n \cdot 2^{k+2}, 2^{k+1}-1\right)$. It will be clear from the context whether we refer to $\circ$ defined on $[0, \infty)$ or on $\mathbb{N}^{\mathbb{N}}$.

We will use the following properties of the ()o-operator:

Lemma $4.6([25]) . \quad$ (1) If $x \in[0, \infty)$, then $(x)$ 。 is a representative of $x$ in the sense of the representation of real numbers described in Section 2.

(2) If $x, y \in[0, \infty)$ and $x \leq y$ (in the sense of $\mathbb{R})$, then $(x)_{\circ} \leq \mathbb{R}(y) \circ$ and also $(x) \circ \leq_{1}(y) \circ$ (i.e. $\left.\forall n \in \mathbb{N}((x) \circ(n) \leq(y) \circ(n))\right)$.

(3) If $x \in[0, \infty)$, then $(x)$ 。 is monotone, i.e. $\forall n \in \mathbb{N}\left((x) \circ(n) \leq_{0}(x) \circ(n+1)\right)$.

(4) If $x, y \in[0, \infty)$ and $x \leq y$ (in the sense of $\mathbb{R}$ ), then $(y) \circ s$-maj $j_{1}(x)$ 。

Proof. (1)-(3) are part of Lemma 2.10 in [25]. (4) follows from (2) and (3).

Definition 4.7. Let $X$ be a nonempty set. The full set-theoretic type structure $\mathcal{S}^{\omega, X}:=\left\langle S_{\rho}\right\rangle_{\rho \in \mathbf{T}^{X}}$ over $\mathbb{N}$ and $X$ is defined by

$$
S_{0}:=\mathbb{N}, \quad S_{X}:=X, \quad S_{\rho \rightarrow \tau}:=S_{\tau}^{S_{\rho}} .
$$

Here $S_{\tau}^{S_{\rho}}$ is the set of all set-theoretic functions $S_{\rho} \rightarrow S_{\tau}$.

Using this and the ()o-operator we state the following definition:

Definition 4.8. We say that a sentence of $\mathcal{L}\left(\mathcal{A}^{\omega}[X, d, W]_{-b}\right)$ holds in a nonempty hyperbolic space $(X, d, W)$ if it holds in the models ${ }^{8}$ of $\mathcal{A}^{\omega}[X, d, W]_{-b}$ obtained by letting the variables range over the appropriate universes of the full set-theoretic type structure $\mathcal{S}^{\omega, X}$ with the set $X$ as the universe for the base type $X, 0_{X}$ is interpreted by an arbitrary element of $X, W_{X}\left(x, y, \lambda^{1}\right)$ is interpreted as $W\left(x, y, r_{\tilde{\lambda}}\right)$, where $r_{\tilde{\lambda}} \in[0,1]$ is the unique real number represented by $\tilde{\lambda}^{1}$ and $d_{X}$ is interpreted as $d_{X}(x, y):={ }_{1}(d(x, y))_{\text {。 }}$.

Analogously for $\mathcal{L}\left(\mathcal{A}^{\omega}[X, d]_{-b}\right)$ and nonempty metric spaces $(X, d)$ where the interpretation of $W_{X}$ is dropped.

Finally, we define a maximum functional, which is particularly useful for constructing majorants for functionals of degree 1.

Definition 4.9 ([25]). For $\rho=\rho_{1} \rightarrow \ldots \rightarrow \rho_{k} \rightarrow 0 \in \mathbf{T}$ we define $\max _{\rho}$ by

$$
\max _{\rho}(x, y):=\lambda v_{1}^{\rho}, \ldots, v_{k}^{\rho_{k}} \cdot \max _{\mathbb{N}}\{x \underline{v}, y \underline{v}\} .
$$

For types $0 \rightarrow \rho$ with $\rho=\rho_{1} \rightarrow \ldots \rightarrow \rho_{k} \rightarrow 0$, we define functionals $(\cdot)^{M}$ of types $(0 \rightarrow \rho) \rightarrow 0 \rightarrow \rho$ by :

$$
x^{M}\left(y^{0}\right):=\lambda \underline{v} \underline{\rho} \cdot \max _{\mathbb{N}}\{x(i, \underline{v}) \mid i=1, \ldots, y\} .
$$

We now state the main version of our metatheorem for unbounded metric, hyperbolic and $\mathrm{CAT}(0)$-spaces:

Theorem 4.10. (1) Let $\rho$ be of degree $(1, X)$ or 2 and let $B_{\forall}(x, u)$, resp. $C_{\exists}(x, v)$, be $\forall$-, resp. $\exists$-formulas that contain only $x, u$ free, resp. $x, v$ free. Assume that the constant $0_{X}$ does not occur in $B_{\forall}, C_{\exists}$ and that

$$
\mathcal{A}^{\omega}[X, d]_{-b} \vdash \forall x^{\rho}\left(\forall u^{0} B_{\forall}(x, u) \rightarrow \exists v^{0} C_{\exists}(x, v)\right) .
$$

Then there exists a partial functional $\Phi: S_{\widehat{\rho}} \rightarrow \mathbb{N}$ whose restriction to the strongly majorizable elements $M_{\widehat{\rho}}$ of $S_{\widehat{\rho}}{ }^{9}$ is a total (bar recursively [40])

\footnotetext{
${ }^{8}$ Here we use the plural since the interpretation of $0_{X}$ is not uniquely determined.

${ }^{9}$ In the sense of [1].
} 
computable functional ${ }^{10}$ and the following holds in all nonempty metric spaces $(X, d)$ : for all $x \in S_{\rho}, x^{*} \in S_{\widehat{\rho}}$ if there exists an $a \in X$ s.t. $x^{*} \gtrsim^{a} x$, then ${ }^{11}$

$$
\forall u \leq \Phi\left(x^{*}\right) B_{\forall}(x, u) \rightarrow \exists v \leq \Phi\left(x^{*}\right) C_{\exists}(x, v) .
$$

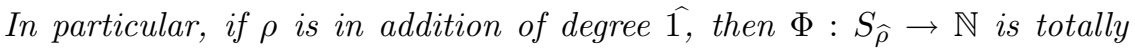
computable.

If $0_{X}$ does occur in $B_{\forall}$ and/or $C_{\exists}$, then the bound $\Phi$ depends (in addition to $\left.x^{*}\right)$ on an upper bound $\mathbb{N} \ni n \geq d\left(0_{X}, a\right)$.

(2) The theorem also holds for $\mathcal{A}^{\omega}[X, d, W]_{-b}$ and nonempty hyperbolic spaces $(X, d, W)$ as well as for $\mathcal{A}^{\omega}[X, d, W, \mathrm{CAT}(0)]_{-b}$ and nonempty $\mathrm{CAT}(0)$ spaces $(X, d, W)$.

Instead of single variables $x, u, v$ and single premises $\forall u B_{\forall}(x, u)$ we may have tuples of variables and finite conjunctions of premises. In the case of a tuple $\underline{x}$ we then have to require that we have a tuple $\underline{x}^{*}$ of a-majorants for a common a $\in X$ for all the components of the tuple $\underline{x}$.

Theorem 4.10 will be proved in section 9 below.

Remark 4.11. The proof of Theorem 4.10 actually provides an algorithm for the extraction of $\Phi$ from a given proof. The complexity of $\Phi$ depends on the principles used in the proof. E.g. if the axiom of dependent choice is not used $\Phi$ will be a primitive recursive functional (in the sense of [10]). Moreover, we then can allow arbitrary types $\rho$ in the parameters (with majorants of type $\widehat{\rho}$ ) since the restriction to types of degree $(1, X)$ or 2 is made necessary only by the interpretation of dependent choice using bar recursive functionals.

Remark 4.12. Another way to treat parameters $x^{\rho}, \rho$ of degree $(1, X)$ or 2 , is to require for a majorant a computable functional $t$ in $S_{\underline{\sigma}} \rightarrow S_{\widehat{\rho}}$, ${ }^{12}$ where all $\sigma_{i}$ are of degree 1 . Then we may obtain a totally computable $\Phi: S_{\underline{\sigma}} \rightarrow \mathbb{N}$ such that given $\underline{c} \in S_{\underline{\sigma}}$, if there exists an $a \in X$ for which $t(\underline{c}) \gtrsim_{\rho}^{a} x$, then the bound $\Phi(\underline{c})$ applies.

Remark 4.13. From the proof of Theorem 4.10 (to be given in section 9 below) two further extensions follow: ${ }^{13}$

(1) The language may be extended by $a$-majorizable constants (in particular constants of types 0 and 1 , which are always uniformly majorizable) where the extracted bounds then additionally depend on ( $a$-majorants for) the new constants.

(2) The theory may be extended by purely universal axioms or, alternatively, axioms which can be reformulated into purely universal axioms using new majorizable constants if the types of the quantifiers are all of degree 2 or $(1, X),{ }^{14}$ as purely universal axioms are their own functional interpretation. Again the extracted bounds depend on ( $a$-majorants for) these new constants. Then the conclusion holds in all metric $(X, d)$, resp. hyperbolic

\footnotetext{
${ }^{10}$ In the sense of [16] relativized to the type structure $\mathcal{M}^{\omega}$ of strongly majorizable functionals from [1].

${ }^{11}$ Note that $x^{*} \gtrsim^{a} x$ implies that $x^{*} \operatorname{s}-\operatorname{maj}_{\widehat{\rho}} x^{*}$ and hence the strong majorizability of $x^{*}$ so that $\Phi\left(x^{*}\right)$ is defined.

${ }^{12}$ Since $t$ is of degree 2, the computability of $t$ implies its (strong) majorizability.

${ }^{13}$ Added in proof: based on this remark, Theorem 4.10 has meanwhile also been adapted to $\delta$-hyperbolic spaces (in the sense of Gromov) and $\mathbb{R}$-trees (in the sense of Tits); see [32].

${ }^{14}$ This ensures that validity in $\mathcal{S}^{\omega, X}$ implies validity in $\mathcal{M}^{\omega, X}$ defined further below.
} 
$(X, d, W)$ spaces which satisfy these axioms (under a suitable interpretation of the new constants if any).

Remark 4.14. The need for the restriction to $\exists$-formulas $C_{\exists}$ in Theorem 4.10 is a consequence of the fact that our theories are based on classical logic, where one can already produce counterexamples for formulas $\exists v^{0} \forall w^{0} C_{q f}(v, w)$ with $C_{q f}$ quantifier-free. If one bases the system on intuitionistic logic instead, this can (even in the presence of many ineffective principles) be avoided and effective bounds for formulas $C$ of arbitrary complexity can be extracted (though no longer bounds on universal premises $\left.\forall u^{0} B_{\forall}\right)$. See [9] for this.

As a corollary to the proof of Theorem 4.10 we obtain Theorem 3.7 in [25]:

Corollary 4.15. (1) Let $\sigma, \rho$ be types of degree 1 and $\tau$ be a type of degree $(1, X)$. Let $s^{\sigma \rightarrow \rho}$ be a closed term of $\mathcal{A}^{\omega}[X, d]$ and let $B_{\forall}(x, y, z, u)$, resp. $C_{\exists}(x, y, z, v)$, be $\forall$-resp. $\exists$-formulas that only contain $x, y, z, u$ free, resp. $x, y, z, v$ free. If

$$
\forall x^{\sigma} \forall y \leq_{\rho} s(x) \forall z^{\tau}\left(\forall u^{0} B_{\forall}(x, y, z, u) \rightarrow \exists v^{0} C_{\exists}(x, y, z, v)\right)
$$

is provable in $\mathcal{A}^{\omega}[X, d]$, then one can extract a computable functional $\Phi$ : $\mathcal{S}_{\sigma} \times \mathbb{N} \rightarrow \mathbb{N}$ such that for all $x \in \mathcal{S}_{\sigma}$ and all $b \in \mathbb{N}$

$$
\forall y \leq_{\rho} s(x) \forall z^{\tau}\left[\forall u \leq \Phi(x, b) B_{\forall}(x, y, z, u) \rightarrow \exists v \leq \Phi(x, b) C_{\exists}(x, y, z, v)\right]
$$

holds in any nonempty metric space $(X, d)$ whose metric is bounded by $b \in \mathbb{N}$.

(2) If the premise is proved in ' $\mathcal{A}^{\omega}[X, d, W]$ ', instead of ' $\mathcal{A}^{\omega}[X, d]$ ', then the conclusion holds in all b-bounded hyperbolic spaces.

(3) If the premise is proved in ' $\mathcal{A}^{\omega}[X, d, W, \mathrm{CAT}(0)]$ ', instead of ' $\mathcal{A}^{\omega}[X, d, W]$ ', then the conclusion holds in all b-bounded CAT(0)-spaces.

Instead of single variables $x, y, z, u, v$ we may also have finite tuples of variables $\underline{x}, \underline{y}, \underline{z}, \underline{u}, \underline{v}$ as long as the elements of the respective tuples satisfy the same type of restrictions as $x, y, z, u, v$.

Moreover, instead of a single premise of the form $\forall u^{0} B_{\forall}(x, y, z, u)^{\prime}$ we may have a finite conjunction of such premises.

Remark 4.16. This result (first proved in [25]) was recently strengthened by extending the formal systems by a strong nonstandard uniform boundedness principle $\exists-\mathrm{UB}^{X}$; see $[17]$.

Proof. Take $a=0_{X}$. For $x$, which has type $\sigma$ of degree 1 (w.l.o.g. $\sigma=1$ ), we easily see (even using only strong majorization s-maj) that $x^{M} \gtrsim^{0_{X}} x$. Next, for the $0_{X}$-majorant $s^{*} \gtrsim^{0_{X}} s$, which we can construct by induction on the structure of $s$ as a closed term of $\mathcal{A}^{\omega}$ (see Lemma 9.9 in Section 9 ), we have that $s^{*}\left(x^{M}\right) \gtrsim_{1}^{0_{X}} y$ for all $y \leq_{1} s(x)$. Given a bound $b \in \mathbb{N}$ on the metric, let $\tau=\tau_{1} \rightarrow \ldots \rightarrow \tau_{k} \rightarrow X$; then also $\lambda \underline{x}^{\widehat{\underline{T}}} . b \gtrsim_{\tau}^{0_{X}} z$. Likewise, independent of the choice of $a$ we have that the distance $d\left(0_{X}, a\right) \leq b$, but for $a=0_{X}$ even $d\left(0_{X}, a\right)=0$.

Then by Theorem 4.10 we can extract a (bar recursive) functional $\phi$ such that $\phi\left(x^{M}, s^{*}\left(x^{M}\right), \lambda \underline{x} \underline{\underline{\tau}} . b, 0\right)$ is a bound on $\exists v$, resp. $\forall u$, for any $b$-bounded metric space. Since both the functional $(\cdot)^{M}$, the $0_{X}$-majorant $s^{*}$ for $s$ and the $0_{X}$-majorant $\lambda \underline{x}^{\widehat{\tau}} . b$ for $z$ are given by closed terms of $\mathcal{A}^{\omega}$ (and hence primitive recursive in the sense of 
[10]), the functional

$$
\Phi: \equiv \lambda x, b \cdot \phi\left(x^{M}, s^{*}\left(x^{M}\right), \lambda \underline{x}^{\widehat{\widehat{T}}} \cdot b, 0\right)
$$

is computable and yields the desired bound.

Note that in $\mathcal{A}^{\omega}[X, d]$ we have the boundedness of $(X, d)$ as an axiom, while Theorem 4.10 only allows one to treat the boundedness of $(X, d)$ as an implicative assumption. Since (due to the restrictions on our weak extensionality rule) our systems do not satisfy the deduction theorem, ${ }^{15}$ strictly speaking this corollary does not follow from Theorem 4.10, but rather from the proof of Theorem 4.10: As mentioned in Remark 4.13, we may freely add another purely universal axiom, i.e. the axiom that $(X, d)$ is a $b$-bounded metric space, to the theory $\mathcal{A}^{\omega}[X, d]_{-b}$.

Similarly, one can derive Corollary 3.11 from [25], but we will state a generalized version of Corollary 3.11 from [25] below. For most applications to be discussed in this paper the following more concrete version of the metatheorem is sufficient:

Corollary 4.17. Let $P$ (resp. K) be a $\mathcal{A}^{\omega}$-definable Polish (resp. compact) metric space, ${ }^{16}$ let $\tau$ be of degree $\hat{1}$ and let $B_{\forall}$, resp. $C_{\exists}$, be $\forall$-resp. $\exists$-formulas that only contain $x, y, z, u$ free, resp. $x, y, z, v$ free, where furthermore $0_{X}$ does not occur in $B_{\forall}, C_{\exists}$. If

$$
\mathcal{A}^{\omega}[X, d, W]_{-b} \vdash \forall x \in P \forall y \in K \forall z^{\tau}\left(\forall u^{0} B_{\forall} \rightarrow \exists v^{0} C_{\exists}\right),
$$

then there exists a computable functional $\Phi: \mathbb{N}^{\mathbb{N}} \times \mathbb{N}^{(\mathbb{N} \times \ldots \times \mathbb{N})} \rightarrow \mathbb{N}$ s.t. the following holds in every nonempty hyperbolic space $(X, d, W)$ : for all representatives $r_{x} \in \mathbb{N}^{\mathbb{N}}$ of $x \in P$ and all $z \in S_{\tau}, z^{*} \in \mathbb{N}^{(\mathbb{N} \times \ldots \times \mathbb{N})}$ if there exists an $a \in X$ for which $z^{*} \gtrsim_{\tau}^{a} z$, then

$$
\forall y \in K\left(\forall u \leq \Phi\left(r_{x}, z^{*}\right) B_{\forall} \rightarrow \exists v \leq \Phi\left(r_{x}, z^{*}\right) C_{\exists}\right) .
$$

As before, instead of single variables $x, y, z$ and a single premise $\forall u^{0} B_{\forall}$, we may have tuples of variables (for $x, y$ ranging over different Polish, resp. compact metric spaces) and a finite conjunction of premises.

Analogously, for $\mathcal{A}^{\omega}[X, d]_{-b}$ or $\mathcal{A}^{\omega}[X, d, W, \mathrm{CAT}(0)]_{-b}$, where then $(X, d)$, resp. $(X, d, W)$, is an arbitrary nonempty metric resp. CAT(0)-space.

Proof. Using the representation of $P$ and $K$ in $\mathcal{A}^{\omega}$, quantification over $x \in P$ and $y \in K$ can be expressed as a quantification over all $x^{1}$, resp. all $y^{1} \leq s$, for some closed function term $s$. Then, for (type 1-)representatives $r_{x}$ of elements $x$ we have

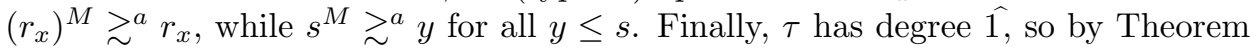
4.10 we obtain a totally computable bound $\Phi\left(r_{x}, z^{*}\right)$.

Remark 4.18. From the proof of the previous corollary it is clear that here and in the results below we can treat parameters $x \in \mathbb{N}$ and $x \in \mathbb{N}^{\mathbb{N}}$ directly (without having to represent these sets as Polish spaces $P$ ). Since rational numbers $q \in \mathbb{Q}_{+}^{*}$ can be encoded by natural numbers, we may also use parameters $x \in \mathbb{Q}_{+}^{*}$.

In Corollary 4.20 below we will single out particularly important special cases where (for a suitable choice of ' $a$ ') a majorant $z^{*}$ of $z$, as assumed to exist in Corollary 4.17, can easily be constructed. For this we need a number of important classes of functions $f: X \rightarrow X$ which we will define next.

\footnotetext{
${ }^{15}$ See $[25]$ for an extensive discussion of this point.

${ }^{16}$ For details on this see [25] and [19].
} 
Definition 4.19. A function $f: X \rightarrow X$ on a metric space $(X, d)$ is called

- nonexpansive (' $f$ n.e.') if $d(f(x), f(y)) \leq d(x, y)$ for all $x, y \in X$,

- quasi-nonexpansive if $\forall p, x \in X(d(p, f(p))=0 \rightarrow d(f(x), f(p)) \leq d(x, p))$,

- weakly quasi-nonexpansive if $\exists p \in X(d(p, f(p))=0 \wedge \forall x \in X(d(f(x), f(p))$ $\leq d(x, p)))$ or, equivalently,

$$
\exists p \in X \forall x \in X(d(f(x), p) \leq d(x, p)) .
$$

- Lipschitz continuous if $d(f(x), f(y)) \leq L \cdot d(x, y)$ for some $L>0$ and for all $x, y \in X$,

- Hölder-Lipschitz continuous if $d(f(x), f(y)) \leq L \cdot d(x, y)^{\alpha}$ for some $L>0$, $0<\alpha \leq 1$ and for all $x, y \in X$.

For normed linear spaces $(X,\|\cdot\|)$ those definitions are to be understood w.r.t. the induced metric $d(x, y):=\|x-y\|$.

The notion of quasi-nonexpansivity was introduced by Dotson in [6], and the notion of weak quasi-nonexpansivity is (implicitly) due to B. Lambov and the second author [26] (note that in contexts where quasi-nonexpansive mappings are used it is always assumed that fixed points exist so that 'weakly quasi-nonexpansive' is indeed weaker than 'quasi-nonexpansive'). ${ }^{17}$ Using that $\leq_{\mathbb{R}}$ and $=_{\mathbb{R}}$ are $\Pi_{1}^{0}$-statements, we observe that the above statements, except for ' $f$ quasi-nonexpansive' and ' $f$ weakly quasi-nonexpansive', can - when formalized in $\mathcal{L}\left(\mathcal{A}^{\omega}[X, d, W]\right)$ - be written as $\forall$ formulas, where in the case of Lipschitz and Hölder-Lipschitz the constants $L$, resp. $L$ and $\alpha$, are assumed to be given as parameters. For ' $f$ weakly quasi-nonexpansive', if we take the fixed point $p$ as a parameter, the remaining formula can be written as a $\forall$-formula, so that to use ' $f$ weakly quasi-nonexpansive' as a premise one needs to quantify over the additional parameter $p$. The statement ' $f$ quasi-nonexpansive' is of the form $\forall \rightarrow \forall$ and hence not of a suitable form to serve as a premise, if we want to apply our metatheorems. Most theorems involving quasi-nonexpansive functions easily extend to the 'weakly quasi-nonexpansive' functions which makes our metatheorems applicable. For examples of this see [26].

As examples of weakly quasi-nonexpansive functions (communicated by L. Leustean) consider in the setting of normed linear spaces (with a convex subset $C$ ) the class of functions satisfying $\|f(x)\| \leq\|x\|$, which are weakly quasinonexpansive in the fixed point $0_{X}$. To see that such functions need not be quasinonexpansive consider $f:[0,1] \rightarrow[0,1]$ (on the convex subset $[0,1]$ of $\mathbb{R}$ ) defined by $f(x):=x^{2}$, which has fixed points 0,1 , but is only weakly quasi-nonexpansive in 0 .

For unbounded hyperbolic spaces $(X, d, W)$ we now state the following corollary:

Corollary 4.20. (1) Let $P$ (resp. $K$ ) be a $\mathcal{A}^{\omega}$-definable Polish (resp. compact) metric space and $B_{\forall}, C_{\exists} \forall$-resp. $\exists$-formulas. Assume we can prove in $\mathcal{A}^{\omega}[X, d, W]_{-b}$ a sentence:

$$
\forall x \in P \forall y \in K \forall z^{X} \forall f^{X \rightarrow X}\left(f \text { n.e. } \wedge \forall u^{0} B_{\forall}(x, y, z, f, u) \rightarrow \exists v^{0} C_{\exists}(x, y, z, f, v)\right),
$$

where $0_{X}$ does not occur in $B_{\forall}$ and $C_{\exists}$.

\footnotetext{
${ }^{17}$ The concept of weakly quasi-nonexpansive mapping has recently been formulated independently - under the name of $J$-type mapping - in [8] where the fixed point $p$ is called a 'center'.
} 
Then there exists a computable functional $\Phi: \mathbb{N}^{\mathbb{N}} \times \mathbb{N} \rightarrow \mathbb{N}$ s.t. for all representatives $r_{x} \in \mathbb{N}^{\mathbb{N}}$ of $x \in P$ and all $b \in \mathbb{N}$

$\forall y \in K \forall z^{X} \forall f X \rightarrow X\left(f\right.$ n.e. $\wedge d_{X}(z, f(z)) \leq_{\mathbb{R}}(b)_{\mathbb{R}}$

$$
\left.\wedge \forall u^{0} \leq \Phi\left(r_{x}, b\right) B_{\forall}(x, y, z, f, u) \rightarrow \exists v^{0} \leq \Phi\left(r_{x}, b\right) C_{\exists}(x, y, z, f, v)\right)
$$

holds in all nonempty hyperbolic spaces $(X, d, W)$. The analogous statements holds for for $\mathcal{A}^{\omega}[X, d, W, \mathrm{CAT}(0)]_{-b}$ and $\mathrm{CAT}(0)$-spaces $(X, d, W)$.

(2) The corollary also holds for an additional parameter $\forall z^{\prime} X$ if we add the additional premise $d_{X}\left(z, z^{\prime}\right) \leq_{\mathbb{R}}(b)_{\mathbb{R}}$ to the conclusion.

(3) Furthermore, the corollary holds for an additional parameter $\forall c^{0 \rightarrow X}$ if one adds the premise $\forall n\left(d_{X}(z, c(n)) \leq_{\mathbb{R}}(b)_{\mathbb{R}}\right)$ or just $\forall n\left(d_{X}(z, c(n)) \leq_{\mathbb{R}}\right.$ $\left.(g(n))_{\mathbb{R}}\right)$ to the conclusion, where the bound then additionally depends on $g: \mathbb{N} \rightarrow \mathbb{N}$.

(4) Statements (1), (2) and (3) also hold if we replace ' $f$ n.e.' with ' $f$ Lipschitz continuous', 'f Hölder-Lipschitz continuous' (with $L, \alpha \in \mathbb{Q}_{+}^{*}$, where $\alpha \leq 1$, as parameters) or ' $f$ uniformly continuous' (with a modulus of uniform continuity $\omega: \mathbb{N} \rightarrow \mathbb{N}$ as a type-1 parameter). For Lipschitz and LipschitzHölder continuous functions the bound additionally depends on the new parameters $L$, resp. $L, \alpha$, and for uniformly continuous functions the bound additionally depends on a given modulus of uniform continuity $\omega: \mathbb{N} \rightarrow \mathbb{N}$.

(5) Furthermore, (1), (2) and (3) hold if we replace ' $f$ n.e.' with ' $f$ weakly quasi-nonexpansive'. For weakly quasi-nonexpansive functions (with fixed point $p$ ) we need to replace the premise ' $d_{X}(z, f(z)) \leq_{\mathbb{R}}(b)_{\mathbb{R}}$ ' by ' $d_{X}(z, p)$ $\leq_{\mathbb{R}}(b)_{\mathbb{R}}$ in the conclusion.

(6) Moreover, (1), (2) and (3) hold if in the conclusion $f$ satisfies ' $d_{X}(z, f(z))$ $\leq_{\mathbb{R}}(b)_{\mathbb{R}}$ ' and if instead of ' $f$ n.e.' we assume

$$
\forall n^{0}, z_{1}^{X}, z_{2}^{X}\left(d_{X}\left(z_{1}, z_{2}\right)<_{\mathbb{R}}(n)_{\mathbb{R}} \rightarrow d_{X}\left(f\left(z_{1}\right), f\left(z_{2}\right)\right) \leq_{\mathbb{R}}\left(\Omega_{0}(n)\right)_{\mathbb{R}}\right),
$$

where $\Omega_{0}$ is a function $\mathbb{N} \rightarrow \mathbb{N}$ treated as a parameter. The bound then depends on $\Omega_{0}$ and $b$.

(7) Finally, (1),(2) and (3) hold if ' $f$ n.e.' is replaced by

$$
\forall n^{0}, \tilde{z}^{X}\left(d_{X}(z, \tilde{z})<_{\mathbb{R}}(n)_{\mathbb{R}} \rightarrow d_{X}(z, f(\tilde{z})) \leq_{\mathbb{R}}(\Omega(n))_{\mathbb{R}}\right),
$$

where $\Omega$ is a function $\mathbb{N} \rightarrow \mathbb{N}$ treated as a parameter. Then we can drop ' $d_{X}(z, f(z)) \leq_{\mathbb{R}}(b)_{\mathbb{R}}$ ' in the conclusion and the extracted bound only depends on $\Omega$ instead of $b$.

Proof. In the following we write for simplicity e.g. ' $d(z, \tilde{z}) \leq n$ ' instead of its formal representation ' $d_{X}(z, \tilde{z}) \leq_{\mathbb{R}}(n)_{\mathbb{R}}$ ' as a formula of $\mathcal{L}\left(\mathcal{A}^{\omega}[X, d]\right)$. For (1), by the comment after Definition 4.19 the premise ' $f$ n.e.' is a $\forall$-formula and hence an admissible premise in Corollary 4.17. The parameters ranging over the Polish metric spaces $P$, resp., compact metric spaces $K$, are treated as before. Choose $a=z$; then trivially $0 \gtrsim^{z} z$ and $\lambda n^{0} \cdot(n+b) \gtrsim^{z} f$, as using $d(z, f(z)) \leq b$ and the nonexpansivity of $f$ and assuming $d(z, \tilde{z}) \leq n$ we get $^{18}$

$$
d(z, f(\tilde{z})) \leq d(z, f(z))+d(f(z), f(\tilde{z})) \leq b+d(z, \tilde{z}) \leq n+b .
$$

For (2) and (3), note that trivially $b \gtrsim^{z} z, z^{\prime}$ and $\lambda n^{0} . b \gtrsim^{z} c$, resp. $g^{M} \gtrsim^{z} c$.

\footnotetext{
${ }^{18}$ Here and in the following we simply write for better readability $d$ and $b$ instead of $d_{X}$ and $(b)_{\mathbb{R}}$, etc.
} 
For (4) and (6), we will show that $d(z, f(z)) \leq b$ in conjunction with the requirement that $f$ is Lipschitz continuous, Hölder-Lipschitz continuous, uniformly continuous or $f$ satisfying (*) allows one to derive an $\Omega$ such that $f$ satisfies (**), thereby reducing these cases to (7). Similarly, if $f$ is weakly quasi-nonexpansive (with fixed point $p$ ) and the premise $d(z, p) \leq b$ is fulfilled, $f$ satisfies $(* *)$. All these conditions on $f$, including $(*)$, can be written as $\forall$-formulas (in the case of ' $f$ weakly quasi-nonexpansive' with a parameter $p$ ) and may hence serve as a premise according to our metatheorem.

For (7), if $f$ satisfies $\forall \tilde{z} \in X(d(z, \tilde{z})<n \rightarrow d(z, f(\tilde{z})) \leq \Omega(n))$, then trivially $\lambda n . \Omega^{M}(n+1) \gtrsim^{z} f$, as $d(z, \tilde{z}) \leq n$ implies $d(z, \tilde{z})<n+1$ which by $(* *)$ implies $d(z, f(\tilde{z})) \leq \Omega(n+1)$. Using the fact that $<_{\mathbb{R}}$ is a $\Sigma_{1}^{0}$-statement and $\leq_{\mathbb{R}}$ is a $\Pi_{1}^{0}$ statement we can express $(* *)$ as a $\forall$-formula. Also note that $f$ satisfying $(* *)$ implies a bound on $d(z, f(z))$ : since $d(z, z)<1$ by $(* *)$ we have $d(z, f(z)) \leq \Omega(1)$.

If $f$ is Lipschitz continuous with constant $L>0$, then one shows, using $d(z, f(z))$ $\leq b$ and the triangle inequality and assuming $d(z, \tilde{z}) \leq n$

$$
d(z, f(\tilde{z})) \leq d(z, f(z))+d(f(z), f(\tilde{z})) \leq L \cdot d(z, \tilde{z})+b \leq L \cdot n+b,
$$

so $f$ satisfies $(* *)$ with $\Omega(n):=\lceil L \cdot n\rceil+b$. If $f$ is Hölder-Lipschitz continuous, i.e. $d(f(x), f(y)) \leq L \cdot d(x, y)^{\alpha}$ for constants $L>0$ and $0<\alpha \leq 1$, then $f$ satisfies $(* *)$ with $\Omega(n):=\left\lceil L \cdot n^{\alpha}\right\rceil+b$.

If $f: X \rightarrow X$ with $(X, d, W)$ a hyperbolic space is uniformly continuous with modulus ${ }^{19} \omega$, then $f$ satisfies $(* *)$ with $\Omega(n):=n \cdot 2^{\omega(0)}+b+1$. Given $z, \tilde{z} \in X$ with $d(z, \tilde{z})<n$ we can (using $W$ with $z, \tilde{z}$ and suitable $\lambda$ to construct $z_{1}, W$ with $z_{1}, \tilde{z}$ and suitable $\lambda$ to construct $z_{2}$, etc.) inductively construct points $z_{1}, \ldots, z_{k-1}$ (with $k=n \cdot 2^{\omega(0)}+1$ ) such that

$$
d\left(z, z_{1}\right), d\left(z_{1}, z_{2}\right), \ldots, d\left(z_{k-1}, \tilde{z}\right)<2^{-\omega(0)}
$$

and hence

$$
d\left(f(z), f\left(z_{1}\right)\right), d\left(f\left(z_{1}\right), f\left(z_{2}\right)\right), \ldots, d\left(f\left(z_{k-1}\right), f(\tilde{z})\right) \leq 1\left(=2^{-0}\right) .
$$

Then by the triangle inequality $d(f(z), f(\tilde{z})) \leq k=n \cdot 2^{\omega(0)}+1$, and another use of the triangle inequality yields $d(z, f(\tilde{z})) \leq d(z, f(z))+d(f(z), f(\tilde{z})) \leq n \cdot 2^{\omega(0)}+b+1$.

For weakly quasi-nonexpansive functions $f$ - with fixed point $p$ and with the premise ' $d(z, p) \leq b$ ' - the function $f$ satisfies $(* *)$ with $\Omega(n):=n+2 b$, as given $d(z, \tilde{z})<n$

$$
\begin{aligned}
d(z, f(\tilde{z})) & \leq d(z, p)+d(f(\tilde{z}), p) \leq d(z, p)+d(\tilde{z}, p) \\
& \leq d(z, p)+d(\tilde{z}, z)+d(z, p) \leq n+2 b .
\end{aligned}
$$

Alternatively, choosing $a=p$ and writing (**) with $p$ instead of $z$ (and adjusting the other majorants accordingly) $f$ even satisfies $(* *)$ with $\Omega(n):=n$, as given $d(p, \tilde{z})<n$

$$
d(p, f(\tilde{z})) \leq d(p, \tilde{z}) \leq n .
$$

If $f$ satisfies $d(z, f(z)) \leq b$ and $(*)$, then given $d(z, \tilde{z})<n$,

$$
d(z, f(\tilde{z})) \leq d(z, f(z))+d(f(z), f(\tilde{z})) \leq \Omega_{0}(n)+b
$$

and hence $f$ satisfies $(* *)$ with $\Omega(n):=\Omega_{0}(n)+b$.

The results then follow using Corollary 4.17 together with Remark 4.18.

\footnotetext{
${ }^{19}$ Recall, that $f: X \rightarrow X$ uniformly continuous with modulus $\omega: \mathbb{N} \rightarrow \mathbb{N}$ is defined as $\forall x, y \in X \forall k \in \mathbb{N}\left(d(x, y)<2^{-\omega(k)} \rightarrow d(f(x), f(y)) \leq 2^{-k}\right)$.
} 
Note, that neither the space nor the range of $f$ are in any way assumed to be bounded, but still the bound $\Phi$ is highly uniform as it depends only on $b$ (and additional inputs $L, \alpha, \omega, \Omega_{0}$ and $\Omega$ as stated in cases (3)-(7)), but not directly on the points $z, z^{\prime}$, the sequence $c$ or the function $f$.

Remark 4.21. Even if ' $z$ ' does not occur in $B_{\forall}, C_{\exists}$ so that ' $\forall z$ ' is a 'dummy' quantifier, we still need in (1)-(4) and (6) in the conclusion a number $b$ with $b \geq d(z, f(z))$ for some $z$ as this is used in constructing a majorant for $f$. In (5) we could identify $z$ with $p$ (and $b:=1$ say) and construct a $p$-majorant of $f$. In (7) we can construct an $f$-majorant without reference to $b$.

Remark 4.22. As the proof of Corollary 4.20 shows, in '(4)' and '(6)-(7)' we may even allow to have the formulas $B_{\forall}, C_{\exists}$ depend on the additional arguments $L, \alpha, \omega$, $\Omega_{0}, \Omega$. The corollary also applies to proofs which use that the parameters are fixed as certain closed terms of $\mathcal{A}^{\omega}$ (and so - in the case of $\omega, \Omega_{0}, \Omega$ - are fixed computable, primitive recursively in the sense of Gödel, functions).

Remark 4.23. Note that for $f$ nonexpansive, Lipschitz, Hölder-Lipschitz or uniformly continuous, $f$ is provably extensional. For $f$ weakly quasi-nonexpansive or $f$ satisfying conditions $(*)$ or $(* *)$ it does not follow that $f$ is extensional. Thus in these cases, if an instance of the extensionality of $f$ is used in a proof, it must either be provable via the extensionality rule (or one must explicitly require $f$ to be (provably) extensional, e.g. by requiring that $f$ is at least uniformly continuous).

Remark 4.24. Except for the case of $f$ being uniformly continuous all results also hold for general (non-hyperbolic) metric spaces $\mathcal{A}^{\omega}[X, d]_{-b},(X, d)$. This also applies to Corollaries 4.26 and 5.2 below. Note that in general metric spaces uniformly continuous functions cannot be majorized, i.e. for (**) no suitable $\Omega(n)$ can be defined, because given $x, y \in X$ we cannot construct intermediate points in order to be able to make use of the uniform continuity of $f$.

For a study of metric spaces for which uniformly continuous functions $f$ admit the definition of a suitable $\Omega$ see [36]. Otherwise, in the setting of metric spaces, we need to require explicitly that a given uniformly continuous function $f$ with modulus $\omega$ also satisfies $(* *)$ with a suitable $\Omega$.

As a generalization of Corollary 3.11 in [25] we prove the following:

Definition 4.25. Let $f: X \rightarrow X$. Then

- for Fix $(f):=\left\{x^{X} \mid x=_{X} f(x)\right\}$ the formula $\operatorname{Fix}(f) \neq \emptyset$ expresses $f$ has a fixed point,

- for $F_{i x}(f, y, b):=\left\{x^{X} \mid d_{X}(x, f(x)) \leq_{\mathbb{R}} \varepsilon \wedge d_{X}(x, y) \leq_{\mathbb{R}} b\right\}$ and $\varepsilon>$ 0 the formula $F_{i x}(f, y, b) \neq \emptyset$ expresses $f$ has an $\varepsilon$-fixed point in a $b$ neighborhood of $y$.

Corollary 4.26. (1) Let $P$ (resp. $K$ ) be a $\mathcal{A}^{\omega}$-definable Polish (resp. compact) metric space and let $B_{\forall}$ and $C_{\exists}$ be as before. If $\mathcal{A}^{\omega}[X, d, W]_{-b}$ proves a sentence

$$
\forall x \in P \forall y \in K \forall z^{X}, f^{X \rightarrow X}\left(f \text { n.e. } \wedge F i x(f) \neq \emptyset \wedge \forall u^{0} B_{\forall} \rightarrow \exists v^{0} C_{\exists}\right),
$$


then there exists a computable functional $\Phi^{1 \rightarrow 0 \rightarrow 0}$ (on representatives $r_{x}$ : $\mathbb{N} \rightarrow \mathbb{N}$ of elements $x$ of $P$ ) s.t. for all $r_{x} \in \mathbb{N}^{\mathbb{N}}, b \in \mathbb{N}$

$\forall y \in K \forall z^{X}, f^{X \rightarrow X}\left(f\right.$ n.e. $\wedge \forall \varepsilon>0 F i x_{\varepsilon}(f, z, b) \neq \emptyset$

$$
\left.\wedge d_{X}(z, f(z)) \leq_{\mathbb{R}}(b)_{\mathbb{R}} \wedge \forall u^{0} \leq \Phi\left(r_{x}, b\right) B_{\forall} \rightarrow \exists v^{0} \leq \Phi\left(r_{x}, b\right) C_{\exists}\right) .
$$

holds in any nonempty hyperbolic space $(X, d, W)$. The analogous statement holds for for $\mathcal{A}^{\omega}[X, d, W, \mathrm{CAT}(0)]_{-b}$ and $\mathrm{CAT}(0)$ spaces $(X, d, W)$. As before we may have several spaces $P$ and $K$ and corresponding tuples of variables $\underline{x}, \underline{y}$.

(2) The corollary also holds if ' $f$ n.e.' is replaced by $f$ Lipschitz continuous, Hölder-Lipschitz continuous or uniformly continuous, where the extracted bound then additionally will depend on the respective constants and moduli.

(3) Considering the premise ' $f$ weakly quasi-nonexpansive', i.e.

$$
\exists p^{X}\left(f(p)=_{X} p \wedge \forall w^{X}\left(d_{X}(f(p), f(w)) \leq_{\mathbb{R}} d_{X}(p, w)\right)\right)
$$

instead of ' $f$ n.e. $\wedge$ Fix $(f) \neq \emptyset$ ' we may weaken this premise to

$$
\begin{aligned}
& \forall \varepsilon>0 \exists p^{X} \\
& \left(d_{X}(f(p), p) \leq_{\mathbb{R}} \varepsilon \wedge d_{X}(z, p) \leq_{\mathbb{R}}(b)_{\mathbb{R}} \wedge \forall w^{X}\left(d_{X}(f(p), f(w)) \leq_{\mathbb{R}} d_{X}(p, w)\right)\right) .
\end{aligned}
$$

(4) Let $\Psi:(X \rightarrow X) \rightarrow X \rightarrow 1$ be a closed term of $\mathcal{A}^{\omega}[X, d, W]_{-b}$ (without $\left.0_{X}\right)$. Then in (1) and (2) instead of ' $F i x(f) \neq \emptyset$ ' we may weaken $\exists p^{X}\left(\Psi(f, p)=_{\mathbb{R}} 0\right)$ ', expressing that $\Psi(f, \cdot)$ has a root $p$, to

$$
\forall \varepsilon>0 \exists p^{X}\left(d_{X}(z, p) \leq_{\mathbb{R}}(b)_{\mathbb{R}} \wedge|\Psi(f, p)| \leq_{\mathbb{R}} \varepsilon\right)^{\prime},
$$

expressing that $\Psi(f, \cdot)$ has $\varepsilon$-roots $p$ which are b-close to $z$ for every $\varepsilon>0$.

Proof. The statement provable by assumption can be written as

$$
\forall x \in P \forall y \in K \forall z^{X}, p^{X}, f^{X \rightarrow X}\left(f \text { n.e. } \wedge f(p)=_{X} p \wedge \forall u^{0} B_{\forall} \rightarrow \exists v^{0} C_{\exists}\right),
$$

where $f(p)=_{X} p$ can be written as $\forall k^{0}\left(d_{X}(p, f(p)) \leq_{\mathbb{R}} 2^{-k}\right)$ and both $d_{X}(p, f(p))$ $\leq_{\mathbb{R}} 2^{-k}$ and $f$ n.e., resp. the other conditions on $f$, are $\forall$-formulas. Note that $p$ does not occur in $B_{\forall}, C_{\exists}$. By Corollary 4.20(2), under the additional (purely universal) premises $d_{X}(z, p), d_{X}(z, f(z)) \leq_{\mathbb{R}}(b)_{\mathbb{R}}$, we extract a functional $\Phi$ s.t. for all $x \in P$ if $r_{x} \in \mathbb{N}^{\mathbb{N}}$ represents $x$, then

$$
\begin{aligned}
\forall y \in K \forall z^{X}, p^{X}, f^{X \rightarrow X}\left(d_{X}(z, p), d_{X}(z, f(z)) \leq_{\mathbb{R}}(b)_{\mathbb{R}} \wedge f\right. \text { n.e. } \\
\left.\wedge d_{X}(f(p), p) \leq_{\mathbb{R}} 2^{-\Phi\left(r_{x}, b\right)} \wedge \forall u^{0} \leq \Phi\left(r_{x}, b\right) B_{\forall} \rightarrow \exists v^{0} \leq \Phi\left(r_{x}, b\right) C_{\exists}\right)
\end{aligned}
$$

holds in all nonempty hyperbolic spaces $(X, d, W)$ (similarly for the other conditions on $f$, except that then the extracted bound depends on the additional constants and moduli $L, \alpha$ and $\omega)$.

The statement $d_{X}(z, p) \leq_{\mathbb{R}}(b)_{\mathbb{R}} \wedge d_{X}(f(p), p) \leq_{\mathbb{R}} 2^{-\Phi\left(r_{x}, b\right)}$ expresses that $f$ has a $2^{-\Phi\left(r_{x}, b\right)}$-fixed point in a $b$-neighborhood of $z$, which, since $2^{-\Phi\left(r_{x}, b\right)}$ does not depend on $p$, is implied by $\forall \varepsilon>0 F i x_{\varepsilon}(f, z, b) \neq \emptyset$, so (1) and (2) follow from Corollary 4.20 . The weakening of the premise ' $f$ weakly quasi-nonexpansive' in (3) is treated similarly.

For (4), similar to the treatment of $f(p)=_{X} p$ in (1), (2) and (3) we may write $\Psi(f, p)=_{\mathbb{R}} 0$ as $\forall k^{0}\left(|\Psi(f, p)|_{\mathbb{R}} \leq_{\mathbb{R}} 2^{-k}\right)$. Then as before we may weaken this statement to $\forall \varepsilon>0 \exists p^{X}\left(d_{X}(z, p) \leq_{\mathbb{R}}(b)_{\mathbb{R}} \wedge|\Psi(f, p)| \leq_{\mathbb{R}} \varepsilon\right)$. 
Remark 4.27. In the presence of the premise ' $\forall \varepsilon>0 F i x_{\varepsilon}(f, z, b) \neq \emptyset$ ' in the conclusion of (1) one may actually drop the additional premise ' $d_{X}(z, f(z)) \leq_{\mathbb{R}}(b)_{\mathbb{R}}$ ' since $p \in F i x_{1}(f, z, b)$ implies that

$$
\begin{aligned}
& d_{X}(z, f(z)) \leq_{\mathbb{R}} d_{X}(z, p)+_{\mathbb{R}} d_{X}(p, f(p))+_{\mathbb{R}} d_{X}(f(p), f(z)) \\
& \leq_{\mathbb{R}} 2 d_{X}(z, p)+_{\mathbb{R}} d_{X}(p, f(p)) \leq_{\mathbb{R}} 2 b+1 .
\end{aligned}
$$

By more complicated arguments corresponding to the proof of Corollary 4.20(4) and 4.20(5) above, this also applies to (2) and (3) but not to (4).

Remark 4.28. Note that in (1) the original premise ' $F i x(f) \neq \emptyset$ ' is weakened to ' $\forall \varepsilon>0 F i x_{\varepsilon}(f, z, b) \neq \emptyset$ '. By Theorem 1 in [11], nonexpansive mappings on bounded hyperbolic spaces always have $\varepsilon$-fixed points for arbitrary $\varepsilon>0$, while they need not have exact fixed points in general (not even in the case of bounded, closed and convex subsets of Banach spaces such as $c_{0}$; see [39]). Hence, for bounded hyperbolic spaces and nonexpansive mappings the premise ' $\forall \varepsilon>0 F i x_{\varepsilon}(f, z, b) \neq \emptyset$ ' can be dropped if $b$ is taken as an upper bound on the metric $d$. For further discussion, see Remark 3.13 in [25].

\section{HeRBRAND NORMAL FORMS}

The metatheorems in the previous sections allow one to treat at most classical proofs of formulas that prenex to the form $\forall \exists A_{q f}$. Already for the formula class $\Pi_{3}^{0}$, i.e. $\forall x^{0} \exists y^{0} \forall z^{0} A_{q f}(x, y, z)$, there are counterexamples where one can no longer extract effective bounds from a given classical proof. These counterexamples basically correspond to the undecidability of the halting problem for Turing machines.

However, the Herbrand normal form $B^{H}=\forall x^{0} \forall h_{z}^{1} \exists y^{0} A_{q f}\left(x, y, h_{z}(y)\right)$ of $B=$ $\forall x^{0} \exists y^{0} \forall z^{0} A_{q f}(x, y, z)$ does have the appropriate form (and the Herbrand index function $h_{z}$ has a suitable restricted type) to allow the extraction of a bound $\Phi\left(x, h_{z}\right)$ on $\exists y A_{q f}\left(x, y, h_{z}(y)\right)$. Even though $B$ and $B^{H}$ are (ineffectively) equivalent, an extracted bound for $\exists y$ in the Herbrand normal form $B^{H}$ does not yield a bound for $\exists y$ in the original formula $B$, as it may depend, in addition to $x$, on the index function $h_{z}$.

The extraction of bounds for Herbrand normal forms can be generalized to a large class of formulas, more precisely to those for which there exists a prenexation such that the Herbrand index functions are of suitable restricted type. The types of the Herbrand index functions depend on the $\exists \forall$ configurations that occur in the prenexation. A configuration $\exists y^{\rho} \forall z^{\tau}$ gives rise to $\forall h_{z}^{\rho \rightarrow \tau} \exists y^{\rho}$, i.e. Herbrand index functions of type $\rho \rightarrow \tau$. Restricting ourselves to cases where the Herbrand index functions are guaranteed to have majorants, we only allow configurations $\exists y^{\rho} \forall z^{\tau}$ where $\rho=0$ and $\tau$ is of degree $(0, X)$ or 1 . Then the types of the Herbrand index functions are of degree $(0, X)$ or 1 as well. This class of formulas covers all arithmetical formulas as well as many other interesting classes involving the extended types $\mathbf{T}^{X}$.

Clearly, one may extract effective bounds for the Herbrand normal form of formulas if the Herbrand index functions are of suitable restricted type, where naturally, the extracted bounds depend on ( $a$-majorants for) the Herbrand index functions. Of even greater interest is the fact that we may, similar to the result of Corollary 4.26 , weaken or even eliminate some premises of a theorem, even though the conclusion might be of too general a form to allow one to extract effective bounds on $A$ rather than $A^{H}$, as we will show next. 
Definition 5.1. The class $\mathcal{H}$ of formulas consists of all formulas $F$ that have a prenexation $F^{\prime} \equiv \exists x_{1}^{0} \forall y_{1}^{\tau_{1}} \ldots \exists x_{n}^{0} \forall y_{n}^{\tau_{n}} F_{\exists}(\underline{x}, \underline{y})$ where $F_{\exists}$ is an $\exists$-formula and the types $\tau_{i}$ are of degree 1 or $(0, X)$.

We state the following corollary:

Corollary 5.2. (1) Let $P$ (resp. K) be a $\mathcal{A}^{\omega}$-definable Polish (resp. compact) metric space and let the formula $A$ be in the class $\mathcal{H}$, where moreover $A$ does not contain $0_{X}$. If $\mathcal{A}^{\omega}[X, d, W]_{-b}$ proves a sentence

$$
\forall x \in P \forall y \in K \forall z^{X}, f^{X \rightarrow X}(f \text { n.e. } \wedge F i x(f) \neq \emptyset \rightarrow A),
$$

then the following holds in every nonempty hyperbolic space $(X, d, W)$ :

$$
\begin{aligned}
\forall x \in P & \forall y \in K \forall z^{X}, f^{X \rightarrow X} \\
& \left(f \text { n.e. } \wedge \exists b^{0} \forall \varepsilon>0\left(F i x_{\varepsilon}(f, z, b) \neq \emptyset\right) \rightarrow A\right) .
\end{aligned}
$$

The analogous statement holds for $\mathcal{A}^{\omega}[X, d, W, \mathrm{CAT}(0)]_{-b}$ and $\mathrm{CAT}(0)$ spaces $(X, d, W)$. Instead of single spaces $P, K$ we may have several Polish, resp. compact metric, spaces and corresponding tuples $\underline{x}, \underline{y}$ of variables.

(2) The corollary also holds if we replace 'f n.e.' with $f$ Lipschitz continuous, Hölder-Lipschitz continuous or uniformly continuous.

(3) Considering the premise ' $f$ weakly quasi-nonexpansive', i.e.

$$
\exists p^{X}\left(f(p)=_{X} p \wedge \forall w^{X}\left(d_{X}(f(p), f(w)) \leq_{\mathbb{R}} d_{X}(p, w)\right)\right)
$$

instead of ' $f$ n.e. $\wedge F i x(f) \neq \emptyset$ ', we may weaken this premise to

$$
\begin{aligned}
& \exists b^{0} \forall \varepsilon>0 \exists p^{X} \\
& \left(d_{X}(f(p), p) \leq_{\mathbb{R}} \varepsilon \wedge d_{X}(z, p) \leq_{\mathbb{R}}(b)_{\mathbb{R}} \wedge \forall w^{X}\left(d_{X}(f(p), f(w)) \leq_{\mathbb{R}} d_{X}(p, w)\right)\right) .
\end{aligned}
$$

(4) Let $\Psi:(X \rightarrow X) \rightarrow X \rightarrow 1$ be a closed term of $\mathcal{A}^{\omega}[X, d, W]_{-b}$ (without $0_{X}$ ). Then in (1) and (2) instead of 'Fix $(f) \neq \emptyset$ ' we may weaken $\exists p^{X} \Psi(f, p)=_{\mathbb{R}} 0$ ', expressing that $\Psi(f, \cdot)$ has a root in $p$, to $\exists b^{0} \forall \varepsilon>$ $0 \exists p^{X}\left(d_{X}(z, p) \leq_{\mathbb{R}}(b)_{\mathbb{R}} \wedge|\Psi(f, p)| \leq_{\mathbb{R}} \varepsilon\right)$ ', expressing that $\Psi(f, \cdot)$ has $\varepsilon$-roots $p$ which are $b$-close to $z$ for every $\varepsilon>0$.

Proof. Since $A \rightarrow A^{H}$ is logically valid, the statement

$$
\forall x \in P \forall y \in K \forall z^{X}, f^{X \rightarrow X}(f \text { n.e. } \wedge \text { Fix }(f) \neq \emptyset \rightarrow A)
$$

trivially implies

$$
\forall x \in P \forall y \in K \forall z^{X}, f^{X \rightarrow X}\left(f \text { n.e. } \wedge F i x(f) \neq \emptyset \rightarrow A^{H}\right)
$$

where $A^{H}$ is the Herbrand normal form of a suitable prenexation of $A$ as suggested by the formula class $\mathcal{H}$. Pulling outside the universal quantifiers in $A^{H}$, which range over the Herbrand index functions, the statement now has a suitable form, and the index functions have a suitable type to make possible the extraction of an effective numerical bound (by Corollary 4.26) on the numerical universal quantifiers in the premise and existential quantifiers in the conclusion.

As is to be expected, the extracted bound depends on the parameter $x$ via a representative $r_{x}$, on a bound $b \geq d(z, f(z))$ (and $b \geq d\left(z, 0_{X}\right)$ if $0_{X}$ occurs in $A$ ) and on majorants for the Herbrand index functions. Such majorants always exist, as the Herbrand index functions $h$ all are of type degree 1, in which case $h^{M}$ is an $a$-majorant, or of type degree $(0, X)$, i.e. basically a sequence of elements in $X$, in which case we (ineffectively) choose as an $a$-majorant $h^{*}$ any nondecreasing sequence of numbers such that $h^{*}(n) \geq d(h(m), a)$ for all $n \in \mathbb{N}$ and all $m \leq n$, 
e.g. we may take $h^{*}:=\tilde{h}^{M}$, where $\tilde{h}(n):=\lceil d(h(n), a)\rceil$. As before, using the representation of $P$ and $K$ in $\mathcal{A}^{\omega}$, we obtain majorants for (representatives of) $x$ and $y$.

Thus, by Theorem 4.10 and reasoning as in the proof of Corollary 4.26 we may weaken the universal premise ' $F i x(f) \neq \emptyset$ ' to ' $\forall \varepsilon>0 F i x_{\varepsilon}(f, z, b) \neq \emptyset$ '. Shifting the quantifiers ranging over the Herbrand index functions back in, we obtain:

$$
\begin{aligned}
\forall x \in P & \forall y \in K \forall z^{X}, f^{X \rightarrow X} \forall b^{0} \\
& \left(f \text { n.e. } \wedge \forall \varepsilon>0 F i x_{\varepsilon}(f, z, b) \neq \emptyset \wedge d_{X}(z, f(z)) \leq_{\mathbb{R}}(b)_{\mathbb{R}} \rightarrow A^{H}\right) .
\end{aligned}
$$

But using that (ineffectively) $A^{H}$ implies back $A$ this yields that

$$
\begin{aligned}
\forall x \in P & \forall y \in K \forall z^{X}, f^{X \rightarrow X} \forall b^{0} \\
& \left(f \text { n.e. } \wedge \forall \varepsilon>0 F i x_{\varepsilon}(f, z, b) \neq \emptyset \wedge d_{X}(z, f(z)) \leq_{\mathbb{R}}(b)_{\mathbb{R}} \rightarrow A\right)
\end{aligned}
$$

holds in all nonempty hyperbolic spaces $(X, d, W)$.

Finally, since here we are not interested in effective bounds but only the (classical) truth of the statement, we may furthermore omit the premise ' $d_{X}(z, f(z)) \leq_{\mathbb{R}}$ $(b)_{\mathbb{R}}$ ': if for a given $z \in X$ and $f: X \in X$ there exists a $b$ such that ' $\forall \varepsilon>$ $0 F i x_{\varepsilon}(f, z, b) \neq \emptyset$ ' holds, then there also exists a $b^{\prime}$ satisfying both premises, as we may simply take $b^{\prime}=\left\lceil\max \left(b, d_{X}(z, f(z))\right)\right\rceil$.

The cases (2),(3) and (4) are treated similarly.

To see that the restrictions on the types of the Herbrand index functions are necessary consider the following counterexample. In $\mathcal{A}^{\omega}[X, d, W]_{-b}$ one trivially proves that:

$$
\forall f^{X \rightarrow X}(f \text { n.e. } \wedge \text { Fix }(f) \neq \emptyset \rightarrow F i x(f) \neq \emptyset),
$$

where ' $F i x(f) \neq \emptyset$ ' is expressed by ' $\exists z^{X} \forall k^{0}\left(d_{X}(z, f(z)) \leq_{\mathbb{R}} 2^{-k}\right)$ '.

Without the restrictions on the types of the Herbrand index functions in $A^{H}$ and hence on $A$, Corollary 5.2 would allow us to weaken the premise ' $f$ has a fixed point' to ' $f$ has $\varepsilon$-fixed points' and in the case of bounded hyperbolic spaces even eliminate the premise completely since nonexpansive mappings on bounded hyperbolic spaces always have approximate fixed points. Hence we could prove that for the bounded hyperbolic case every nonexpansive mapping has exact fixed points. As we mentioned already, this is false even for bounded closed convex subsets of Banach spaces, such as e.g. $c_{0}$.

This counterexample is ruled out by the restrictions on the types of the Herbrand index functions. Since the statement ' $f$ has a fixed point' is expressed by $\exists z^{X} \forall k^{0}\left(d_{X}(z, f(z)) \leq_{\mathbb{R}} 2^{-k}\right)$, the resulting Herbrand index functions have the type $X \rightarrow 0$. But already this very simple type is not allowed in the formula class $\mathcal{H}$ since $z$ has type $X$ and not 0 . Hence our corollary does not apply.

\section{Metatheorems For NORMED Linear spaCes}

We now discuss the setting of (real) normed linear spaces with convex subsets $C$. As discussed in Machado [34], one may characterize convex subsets of normed spaces in the setting of hyperbolic spaces in terms of additional conditions on the function $W$. The additional conditions are (I) that the convex combinations do not depend on the order in which they are carried out, and (II) that the distance is homothetic. These additional conditions are:

$$
\text { (I) } \begin{aligned}
\forall x, y, z \in X \forall \lambda_{1}, \lambda_{2}, \lambda_{3} \in[0,1]\left(\lambda_{1}+\lambda_{2}+\lambda_{3}={ }_{\mathbb{R}} 1 \rightarrow\right. \\
\left.W\left(z, W\left(y, x, \frac{\lambda_{1}}{1-\lambda_{3}}\right), 1-\lambda_{3}\right)=W\left(x, W\left(z, y, \frac{\lambda_{2}}{1-\lambda_{1}}\right), 1-\lambda_{1}\right)\right),
\end{aligned}
$$


(II) $\forall x, y, z \in X \forall \lambda \in[0,1](d(W(z, x, \lambda), W(z, y, \lambda))=\lambda \cdot d(x, y))$.

The formal version of axiom (I) will look slightly different, as expressing the axiom with $\lambda_{1}+\lambda_{2}+\lambda_{3}=\mathbb{R} 1$ (with the equality as a premise) is problematic for our purposes. Equality on the reals is a universal statement, and hence the axiom itself would no longer be purely universal.

Instead, given $\lambda_{1}, \lambda_{2}$ we may explicitly define $\overline{\lambda_{1}}, \overline{\lambda_{2}}$ and $\overline{\lambda_{3}}$ s.t. provably (in $\mathcal{A}^{\omega}$ ) both $\overline{\lambda_{1}}+\overline{\lambda_{2}}+\bar{\lambda}_{3}=\mathbb{R} 1$ and if $\lambda_{i} \in[0,1]$ and $\lambda_{1}+\lambda_{2}+\lambda_{3}=\mathbb{R} 1$, then $\overline{\lambda_{i}}=\lambda_{i}$ for $i=1,2,3$. The formal versions of the axioms are then as follows:

(I) $\forall x^{X}, y^{X}, z^{X} \forall \lambda_{1}^{1}, \lambda_{2}^{1}$

$\left(W_{X}\left(z, W_{X}\left(y, x, \frac{\overline{\lambda_{1}}}{1-\lambda_{3}}\right), 1-\overline{\lambda_{3}}\right)=W_{X}\left(x, W_{X}\left(z, y, \frac{\overline{\lambda_{2}}}{1-\lambda_{1}}\right), 1-\overline{\lambda_{1}}\right)\right)$,

where $\overline{\lambda_{1}}={ }_{1} \tilde{\lambda_{1}}, \overline{\lambda_{2}}={ }_{1} \min _{\mathbb{R}}\left(\tilde{\lambda_{2}}, 1-{ }_{\mathbb{R}} \overline{\lambda_{1}}\right)$ and $\overline{\lambda_{3}}={ }_{1} 1-{ }_{\mathbb{R}}\left(\overline{\lambda_{1}}+{ }_{\mathbb{R}} \overline{\lambda_{2}}\right)$,

(II) $\forall x^{X}, y^{X}, z^{X} \forall \lambda^{1}\left(d_{X}\left(W_{X}(z, x, \lambda), W_{X}(z, y, \lambda)\right)==_{\mathbb{R}} \tilde{\lambda} \cdot \mathbb{R}_{\mathbb{R}} d_{X}(x, y)\right)$,

where $\tilde{\lambda}$ is the construction in Definition 2.4. As discussed for the other $(X, d, W)$ axioms in Remark 2.6, the axiom (II) is formulated with $W_{X}$ to implicitly satisfy $W_{X}(x, y, \lambda)=_{X} W_{X}(x, y, \tilde{\lambda})$.

Thus, theorems concerning convex subsets of normed linear spaces which can be formalized in $\mathcal{A}^{\omega}[X, d, W]_{-b}+$ Machado's two additional axioms can already be treated using the above Theorem 4.10 (as discussed in Remark 4.13). However, as discussed in [25], metatheorems covering normed linear spaces in general rather than just convex subsets of normed linear spaces can be expected to have many more applications than the applications in fixed point theory investigated so far.

For the new metatheorem for normed linear spaces (with convex subset $C$ ) there are, compared to the new metatheorems for (unbounded) metric spaces, two differences: (1) we fix the choice $a=0_{X}$ and (2) one cannot meaningfully differentiate between $0_{X}$ occurring or not occurring in the theorem to be treated by the metatheorem, since it implicitly occurs whenever the norm is used as the latter measures the distance from $0_{X}$ (in metric spaces, the only purpose of the constant $0_{X}$ was to witness the nonemptiness of the space by a closed term). It is this link between the constant $0_{X}$ and the other constants of normed linear spaces that lets us choose $a=0_{X}$ (one could also use an arbitrary $a$, but then the majorant of the norm would depend on $a$, i.e. the norm is - in contrast to the metric - not uniformly majorizable).

As in [25], the type $C$ for the convex subset $C$ and quantification over elements of types involving $C$ are defined notions, i.e. an element $x \in X$ is of type $C$ if $\chi_{C}(x)={ }_{0} 0$, where $\chi_{C}$ is a constant of type $X \rightarrow 0$ representing the characteristic function of $C$. Note, however, that our weakly extensional context does not allow us to prove that $x=_{X} y \wedge \chi_{C}(x)={ }_{0} 0 \rightarrow \chi_{C}(y)=_{0} 0$, but only if $s=_{X} t$ is provable then $\chi_{C}(s)={ }_{0} \chi_{C}(t)$ (see [25] for a discussion of this point).

Quantification is treated using the following abbreviations:

$$
\begin{array}{ll}
\forall x^{C} A(x) & : \equiv \forall x^{X}\left(\chi_{C}\left(x^{X}\right)=_{0} 0 \rightarrow A(x)\right), \\
\forall f^{1 \rightarrow C} A(f) & : \equiv \forall f^{1 \rightarrow X}\left(\forall y^{1}\left(\chi_{C}(f(y))={ }_{0} 0\right) \rightarrow A(f)\right), \\
\forall f^{X \rightarrow C} A(f) & : \equiv \forall f^{X \rightarrow X}\left(\forall y^{X}\left(\chi_{C}(f(y))={ }_{0} 0\right) \rightarrow A(f)\right) \\
\forall f^{C \rightarrow C} A(f) & : \equiv \forall f^{X \rightarrow X}\left(\forall x^{X}\left(\chi_{C}(x)={ }_{0} 0 \rightarrow \chi_{C}(f(x))={ }_{0} 0\right) \rightarrow A(\tilde{f})\right),
\end{array}
$$

where $\tilde{f}(x)=\left\{\begin{array}{l}f(x), \text { if } \chi_{C}(x)={ }_{0} 0, \\ c_{X}, \text { otherwise. }\end{array}\right.$

Analogously, for the corresponding $\exists$-quantifiers with ' $\wedge$ ' instead of ' $\rightarrow$ '. 
Note that the additional premises to the conclusion are $\forall$-formulas if we have parameters of these defined types. This extends to types of degree $(1, X, C)$ where $\rho$ is of degree $(1, X, C)$ if it has the form $\tau_{1} \rightarrow \ldots \rightarrow \tau_{k} \rightarrow C$, where $\tau_{i}$ has degree 1 or equals $X$ or $C$.

Also note that if we write ' $f$ nonexpansive' for a function $f: C \rightarrow C$, this is to be understood as the $\forall$-formula

$$
\forall x^{X}, y^{X}\left(\chi_{C}(x)={ }_{0} 0={ }_{0} \chi_{C}(y) \rightarrow\|f(x)-f(y)\|_{X} \leq_{\mathbb{R}}\|x-y\|_{X}\right) .
$$

Analogously, for the other notions in Definition 4.19.

Note that by construction it is clear that $A(\tilde{f})$ is (when interpreted in $\mathcal{S}^{\omega, X}$ ) extensional in $f$ w.r.t.

$$
f_{1}={ }_{C \rightarrow C} f_{2}: \equiv \forall x^{X}\left(\chi_{C}(x)={ }_{0} 0 \rightarrow f_{1}(x)={ }_{X} f_{2}(x)\right)
$$

for functions $f_{i}$ satisfying

$$
\forall x^{X}\left(\chi_{C}(x)={ }_{0} 0 \rightarrow \chi_{C}\left(f_{i}(x)\right)={ }_{0} 0\right) .
$$

In the following, when writing $\forall f^{C \rightarrow C} A(f)$ we tacitly assume that $A(f)$ is already extensional in this sense, which, in particular, implies that $A(\tilde{f})$ does not depend on the interpretation of $c_{X}$ used in the construction $f \mapsto \tilde{f}$. This is automatically the case for any formula $A(f)$ that arises by formalizing a property of functions $f: C \rightarrow C$.

Remark 6.1. When we aim to treat parameters $f: C \rightarrow C$ in our metatheorems, we need to majorize not $f$, but rather the extension $\tilde{f}$ to a function $X \rightarrow C$. In [25], where only norm-bounded convex subsets $C$ are considered, the extended function $\tilde{f}$ is easily majorized using the $b$-boundedness of $C$ (as are parameters of type $(1, X, C)$ in general). In this paper, where we consider unbounded convex subsets $C$, majorization must employ special properties of the function $f$, such as e.g. $f$ being nonexpansive. However, the extension $\tilde{f}$ does not in general inherit such properties from $f$, so instead a majorant for $\tilde{f}$ in general will result from deriving a majorant for $f$ on $C$ from special properties of $f$, deriving a majorant for $\tilde{f}$ on $X \backslash C$ from the definition of $\tilde{f}$ and taking the maximum over these two majorants.

Definition 6.2. We say that a sentence of $\mathcal{L}\left(\mathcal{A}^{\omega}[X,\|\cdot\|, C]_{-b}\right)$ holds in a nontrivial (real) normed linear space with a nonempty convex subset $C$, if it holds in the models ${ }^{20}$ of $\mathcal{A}^{\omega}[X,\|\cdot\|, C]_{-b}$ obtained by letting the variables range over the appropriate universes of the full set-theoretic type structure $\mathcal{S}^{\omega, X}$ with the sets $\mathbb{N}, X$ as the universes for the base types 0 and $X$. Here $0_{X}$ is interpreted by the zero vector of the linear space $X, 1_{X}$ by some vector $a \in X$ with $\|a\|=1,+_{X}$ is interpreted as addition in $X,-_{X}$ is the inverse of $x$ w.r.t. + in $X, \cdot{ }_{X}$ is interpreted as $\lambda \alpha \in \mathbb{N}^{\mathbb{N}}, x \in X . r_{\alpha} \cdot x$, where $r_{\alpha}$ is the unique real number represented by $\alpha$, and - refers to scalar multiplication in the $\mathbb{R}$-linear space $X$. Finally, $\|\cdot\|_{X}$ is interpreted by $\lambda x \in X .(\|x\|)_{\circ}$. For the nonempty convex subset $C \subseteq X, \chi_{C}$ is interpreted as the characteristic function for $C$ and $c_{X}$ by some arbitrary element of $C$.

\footnotetext{
${ }^{20}$ Again we use the plural, as in the setting of normed linear spaces the interpretation of $1_{X}$ and $c_{X}$ are not uniquely determined.
} 
The new metatheorem for normed linear spaces (to be proved in section 9 below) is:

Theorem 6.3. (1) Let $\rho$ be of degree $(1, X),(1, X, C)$ or 2 and let $B_{\forall}(x, u)$, resp. $C_{\exists}(x, v)$, be $\forall$-resp. $\exists$-formulas that only contain $x, u$ free, resp. $x, v$ free. Assume

$$
\mathcal{A}^{\omega}[X,\|\cdot\|, C]_{-b} \vdash \forall x^{\rho}\left(\forall u^{0} B_{\forall}(x, u) \rightarrow \exists v^{0} C_{\exists}(x, v)\right) .
$$

Then there exists a partial functional $\Phi: S_{\widehat{\rho}} \times \mathbb{N} \rightarrow \mathbb{N}$ s.t. $\Phi$ is defined on all strongly majorizable elements $M_{\widehat{\rho}}$ of $S_{\widehat{\rho}}$ and computable restricted to those, and the following holds in all nontrivial (real) normed linear spaces $(X,\|\cdot\|, C)$ with a nonempty convex subset $C$ : for all $x \in S_{\rho}, x^{*} \in S_{\widehat{\rho}}$ and $n \in \mathbb{N}$ if $x^{*} \gtrsim^{0} x$ and $(n)_{\mathbb{R}} \geq_{\mathbb{R}}\left\|c_{X}\right\|_{X}$, then

$$
\forall u \leq \Phi\left(x^{*}, n\right) B_{\forall}(x, u) \rightarrow \exists v \leq \Phi\left(x^{*}, n\right) C_{\exists}(x, v) .
$$

In particular, if $\rho$ is in addition of degree $\widehat{1}$, then $\Phi: S_{\widehat{\rho}} \times \mathbb{N} \rightarrow \mathbb{N}$ is totally computable.

(2) For (real) uniformly convex spaces with modulus of uniform convexity $\eta$ statement (1) holds with $(X,\|\cdot\|, C, \eta), \mathcal{A}^{\omega}[X,\|\cdot\|, C, \eta]_{-b}$ instead of $(X,\|\cdot\|, C), \mathcal{A}^{\omega}[X,\|\cdot\|, C]_{-b}$, where the extracted bound $\Phi$ additionally depends on $\eta$.

(3) Analogously, for $\mathcal{A}^{\omega}[X,\langle\cdot, \cdot\rangle, C]_{-b}$, nontrivial (real) inner product spaces $(X,\langle\cdot, \cdot\rangle)$ and nonempty convex subsets $C \subseteq X$.

$A s$ in the metric case, instead of single variables $x, u, v$ and single premises $\forall u B_{\forall}(x, u)$ we may have tuples of variables and finite conjunctions of premises.

Remark 6.4. In the case of metric spaces, if $0_{X}$ did not occur in the formula for which we want to extract a bound, the bound did not depend on a bound on the distance between the chosen $a$ and $0_{X}$. This is mainly because the axioms of $\mathcal{A}^{\omega}[X, d]_{-b}$ place no requirements on $0_{X}$. This is not the case for $c_{X}$ and normed linear spaces, as in the theory $\mathcal{A}^{\omega}[X,\|\cdot\|, C]_{-b}$ the constant $c_{X}$ is necessary for the interpretation of one of the axioms (stating the nonemptiness of $C$ ), and hence in general the extracted term may depend on a bound on the norm of $c_{X}$, even though $c_{X}$ does not occur in the formulas $B_{\forall}$ and $C_{\exists}$. However, if $c_{X}$ does not occur in the formulas $B_{\forall}$ and $C_{\exists}$ and we have another parameter $z \in C$ for which we have a bound on the norm, we need not explicitly demand a bound on $\left\|c_{X}\right\|$, since in the model $c_{X}$ may be interpreted by an arbitrary element of $C$ and we may then interpret $c_{X}$ by $z$.

As a corollary we prove Theorem 3.30 in [25].

Corollary 6.5. (1) Let $\sigma$ be of degree 1 and $\rho$ of degree 1 or $(1, X)$ and let $\tau$ be a type of degree $(1, X, C)$. Let $s$ be a closed term of type $\sigma \rightarrow \rho$ and $B_{\forall}, C_{\exists}$ be $\forall$-resp. $\exists$-formulas. If a sentence

$$
\forall x^{\sigma} \forall y \leq_{\rho} s(x) \forall z^{\tau}\left(\forall u^{0} B_{\forall}(x, y, z, u) \rightarrow \exists v^{0} C_{\exists}(x, y, z, v)\right)
$$

is provable in $\mathcal{A}^{\omega}[X,\|\cdot\|, C]$, then one can extract a computable functional $\Phi: S_{\sigma} \times \mathbb{N} \rightarrow \mathbb{N}$ s.t. for all $x \in S_{\sigma}$

$$
\forall y \leq_{\rho} s(x) \forall z^{\tau}\left(\forall u^{0} \leq \Phi(x, b) B_{\forall}(x, y, z, u) \rightarrow \exists v^{0} \leq \Phi(x, b) C_{\exists}(x, y, z, v)\right)
$$


holds in any non-trivial (real) normed linear space $(X,\|\cdot\|)$ and any nonempty b-bounded (in the norm) convex subset $C \subset X$ (with ' $b_{X}$ ' interpreted by ' $b$ ').

(2) For (real) uniformly convex spaces $(X,\|\cdot\|, \eta)$ with modulus of uniform convexity $\eta$ '(1)' holds with $\mathcal{A}^{\omega}[X,\|\cdot\|, C, \eta]$ and $(X,\|\cdot\|, C, \eta)$ instead of $\mathcal{A}^{\omega}[X,\|\cdot\|, C]$ and $(X,\|\cdot\|, C)$. This time $\Phi$ is a computable functional in $x, b$ and a modulus $\eta$ of uniform convexity for $(X,\|\cdot\|)$ (which interprets the constant ' $\eta$ ').

(3) Analogously, for $\mathcal{A}^{\omega}[X,\langle\cdot, \cdot\rangle, C]$, nontrivial (real) inner product spaces $(X,\langle\cdot, \cdot\rangle)$ and nonempty convex subsets $C \subseteq X$.

Instead of single variables $x, y, z, u, v$ we may also have finite tuples of variables $\underline{x}, \underline{y}, \underline{z}, \underline{u}, \underline{v}$ as long as the elements of the respective tuples satisfy the same type of restrictions as $x, y, z, u, v$.

Moreover, instead of a single premise of the form $\forall u^{0} B_{\forall}(x, y, z, u)^{\prime}$ we may have a finite conjunction of such premises.

Proof. As before in the proof of Corollary 4.15, for $x$ we have $x^{M} \gtrsim^{0_{X}} x$ and for $s(x) \geq_{\rho} y$ we get (using Lemma 3.6) that $s^{*}\left(x^{M}\right) \gtrsim^{0_{X}} y$, where $s^{*}$ is some majorant of $s$ (which exists by Lemma 9.11 as a closed term of $\mathcal{A}^{\omega}$ ). Next, given a norm bound $b \in \mathbb{N}$ on $C$, trivially $(b)_{\mathbb{R}} \geq\left\|c_{X}\right\|$ and writing $\tau=\tau_{1} \rightarrow \ldots \rightarrow \tau_{k} \rightarrow C$, then also $\lambda \underline{x} \underline{\underline{\tau}} . b \gtrsim_{\tau}^{0_{X}} z$.

Thus by Theorem 6.3 we can extract a bar recursive functional $\phi$ such that $\phi\left(x^{M}, s^{*}\left(x^{M}\right), \lambda \underline{\underline{\underline{\tau}}} \cdot b, b\right)$ is a bound on $\exists v$, resp. $\forall u$, for any nontrivial real normed linear space and any (nonempty) $b$-bounded convex subset $C$. Since both the functional $(\cdot)^{M}$, the $0_{X}$-majorant $s^{*}$ for $s$ and the $0_{X}$-majorant $\lambda \underline{x}^{\widehat{\tau}} . b$ for $z$ are given by closed terms of $\mathcal{A}^{\omega}$, the functional

$$
\Phi: \equiv \lambda x, b . \phi\left(x^{M}, s^{*}\left(x^{M}\right), \lambda \underline{x}^{\widehat{\underline{x}}} \cdot b, b\right)
$$

is computable and yields the desired bound.

Note that in $\mathcal{A}^{\omega}[X,\|\cdot\|, C]$ we have the boundedness of $C$ as an axiom, while Theorem 6.3 only allows one to treat the boundedness as an implicative assumption. Therefore, as in the proof of Corollary 4.15, this corollary follows from the proof of Theorem 6.3, rather than from the theorem itself.

We furthermore prove the analogue of Corollary 4.20, though with one important difference: it is no longer sufficient to just have a bound on $\|z-f(z)\|,\left\|z-z^{\prime}\right\|$, etc. as in the metric case. Since the choice of $a$ is fixed to $a=0_{X}$ in the normed linear case, we also need a bound on the distance between $z$ and $0_{X}$, i.e. $\|z\|$. Moreover, for the functions $f: C \rightarrow C$ we consider as parameters, the majorization of $f$, or rather of the extension $\tilde{f}: X \rightarrow C$, requires special care (see Remark 6.1).

Corollary 6.6. (1) Let $P$ (resp. K) be a $\mathcal{A}^{\omega}$-definable Polish (resp. compact) metric space and let $B_{\forall}, C_{\exists}$ be $\forall$-resp. $\exists$-formulas. Assume we prove in $\mathcal{A}^{\omega}[X,\|\cdot\|, C]_{-b}$ a sentence

$$
\forall x \in P \forall y \in K \forall z^{C} \forall f^{C \rightarrow C}\left(f \text { n.e. } \wedge \forall u^{0} B_{\forall}(x, y, z, f, u) \rightarrow \exists v^{0} C_{\exists}(x, y, z, f, v)\right),
$$

where $c_{X}$ does not occur in $B_{\forall}$ and $C_{\exists}$. Then there exists a computable functional $\Phi: \mathbb{N}^{\mathbb{N}} \times \mathbb{N} \rightarrow \mathbb{N}$ s.t. for all representatives $r_{x} \in \mathbb{N}^{\mathbb{N}}$ of $x \in P$ and all $b \in \mathbb{N}$

$$
\begin{aligned}
\forall y \in K \forall z^{C} \forall f^{C \rightarrow C}\left(f \text { n.e. } \wedge\|z\|_{X},\|z-f(z)\|_{X} \leq_{\mathbb{R}}(b)_{\mathbb{R}}\right. \\
\left.\wedge \forall u^{0} \leq \Phi\left(r_{x}, b\right) B_{\forall}(x, y, z, f, u) \rightarrow \exists v^{0} \leq \Phi\left(r_{x}, b\right) C_{\exists}(x, y, z, f, v)\right)
\end{aligned}
$$


holds in all nontrivial (real) normed linear spaces $(X,\|\cdot\|)$ and nonempty convex subsets $C$.

Analogously, for uniformly convex spaces $(X,\|\cdot\|, C, \eta)$ and (real) inner product spaces $(X,\langle\cdot, \cdot\rangle)$, where for uniformly convex spaces the bound $\Phi$ additionally depends on a modulus of uniform convexity $\eta$.

(2) The corollary also holds for an additional parameter $\forall z^{\prime C}$, if we add the additional premise $\left\|z-z^{\prime}\right\|_{X} \leq_{\mathbb{R}}(b)_{\mathbb{R}}$ to the conclusion.

(3) Furthermore, the corollary holds for an additional parameter $\forall c^{0 \rightarrow C}$ if we add the additional premise $\forall n\left(\|z-c(n)\|_{X} \leq_{\mathbb{R}}(b)_{\mathbb{R}}\right)$ or just $\forall n\left(\|z-c(n)\|_{X}\right.$ $\left.\leq_{\mathbb{R}}(g(n))_{\mathbb{R}}\right)$ to the conclusion, where the bound then additionally depends on $g: \mathbb{N} \rightarrow \mathbb{N}$.

(4) (1), (2) and (3) also hold if we replace ' $f$ n.e.' with ' $f$ Lipschitz continuous' or 'f Hölder-Lipschitz continuous' (with $L, \alpha \in \mathbb{Q}_{+}^{*}$, where $\alpha \leq 1$, as parameters) or ' $f$ uniformly continuous' (with a modulus $\omega: \mathbb{N} \rightarrow \mathbb{N}$ as parameter). For Lipschitz and Lipschitz-Hölder continuous functions the bound additionally depends on the new parameters $L$, resp. $L, \alpha$, and for uniformly continuous functions the bound additionally depends on a given modulus of uniform continuity $\omega: \mathbb{N} \rightarrow \mathbb{N}$.

(5) Furthermore, (1), (2) and (3) hold if we replace ' $f$ n.e.' with ' $f$ weakly quasi-nonexpansive'. For weakly quasi-nonexpansive functions (with fixed point $p$ ) we need to replace the premise ' $\|z-f(z)\|_{X} \leq_{\mathbb{R}}(b)_{\mathbb{R}}$ ' by ' $\|p\|_{X} \leq_{\mathbb{R}}$ $(b)_{\mathbb{R}}$ 'in the conclusion.

(6) (1), (2) and (3) also hold if we replace ' $f$ n.e.' in the premise and the conclusion by

$\forall n^{0}, z_{1}^{C}, z_{2}^{C}\left(\left\|z_{1}-z_{2}\right\|_{X}<_{\mathbb{R}}(n)_{\mathbb{R}} \rightarrow\left\|f\left(z_{1}\right)-f\left(z_{2}\right)\right\|_{X} \leq_{\mathbb{R}}\left(\Omega_{0}(n)\right)_{\mathbb{R}}\right)$,

where $\Omega_{0}$ is a function $\mathbb{N} \rightarrow \mathbb{N}$ treated as a parameter and the bound additionally depends on $\Omega_{0}$.

(7) Finally, (1), (2) and (3) hold if the previous conditions on $f$ are replaced by

$$
\forall n^{0}, \tilde{z}^{C}\left(\|\tilde{z}\|_{X}<_{\mathbb{R}}(n)_{\mathbb{R}} \rightarrow\|f(\tilde{z})\|_{X} \leq_{\mathbb{R}}(\Omega(n))_{\mathbb{R}}\right),
$$

where $\Omega$ is a function $\mathbb{N} \rightarrow \mathbb{N}$ treated as a parameter and the bound additionally depends on $\Omega$. In this case we can drop the assumption ' $\|z-f(z)\|_{X} \leq$ $(b)_{\mathbb{R}}$ ' in the conclusion whereas ' $\|z\|_{X} \leq(b)_{\mathbb{R}}$ ' has to remain.

Note that $(*),(* *)$ are logically equivalent to $\forall$-formulas.

Again we may have tuples of variables $\underline{x}, \underline{y}$ ranging over various Polish, resp., compact, metric spaces $P, K$.

Proof. This is basically the same proof as the proof of Corollary 4.20, except for two points: (a) as discussed we need to fix $a=0_{X}$ and we need an additional premise, $\|z\| \leq b$ and (b) the $0_{X}$-majorization of $f$ (actually $\tilde{f}$ ) requires extra care. From the definition of $\tilde{f}$ it is obvious that $n \gtrsim_{X}^{0_{X}} f(x)$ for $x \in X \backslash C$ if $n \geq\left\|c_{X}\right\|$. Also note that since we assume $c_{X}$ does not occur in $B_{\forall}$ and $C_{\exists}$ we may, by Remark 6.4 , interpret $c_{X}$ by the parameter $z$ in the model, so that $\left\|c_{X}\right\| \leq b$. Hence, given an $a$-majorant $\lambda n . f^{*}(n) \gtrsim_{X}^{0_{X}} f$ on the convex subset $C$, we obtain the $0_{X^{-}}$ majorant $\lambda n \cdot \max \left(f^{*}(n), b\right)$ for $\tilde{f}$, and thus the extracted bound does not depend on an explicit bound on the norm of $c_{X}$. In the following we may, therefore, focus on $0_{X}$-majorants for $f$ on the convex subset $C$. 
For (1), (2) and (3) we have that $b \gtrsim^{0_{X}} z, 2 b \gtrsim^{0_{X}} z^{\prime}$ and $\lambda n^{0} .2 b \gtrsim^{0_{X}} c$, resp. $\lambda n . g^{M}(n)+b \gtrsim^{0_{X}} c$. For $\lambda n^{0} . n+3 b \gtrsim^{0_{X}} f$, where $f$ is nonexpansive, we reason as follows: assume $\|\tilde{z}\| \leq n$; then

$$
\begin{aligned}
\|f(\tilde{z})\| & =\|f(\tilde{z})-f(z)+f(z)-z+z\| \\
& \leq\|f(\tilde{z})-f(z)\|+\|f(z)-z\|+\|z\| \\
& \leq\|\tilde{z}-z\|+b+b \\
& \leq\|\tilde{z}\|+\|z\|+2 b \\
& \leq n+3 b .
\end{aligned}
$$

Similarly, for (4), (5), (6) and (7) one obtains $\lambda n^{0} \cdot \Omega^{M}(n+1) \gtrsim^{0_{X}} f$ if $\Omega$ satisfies $(* *)$ from (7). As in the metric case, one may obtain a bound on $\|f(z)-z\|$ using $(* *):\|z\| \leq b(\Rightarrow\|z\|<b+1)$ implies $\|f(z)\| \leq \Omega(b+1)$ and hence $\|f(z)-z\| \leq$ $\Omega(b+1)+b$. For (4), (5) and (6) we derive the various $\Omega$ 's, under the assumptions $\|z\|,\|f(z)-z\| \leq b$, as follows:

If $f$ is Lipschitz continuous with constant $L>0$ and we assume $\|\tilde{z}\| \leq n$, then using the triangle inequality and the aforementioned assumptions

$$
\begin{aligned}
\|f(\tilde{z})\| & \leq\|f(\tilde{z})-f(z)\|+\|f(z)-z\|+\|z\| \\
& \leq L \cdot\|\tilde{z}-z\|+b+b \\
& \leq L \cdot(n+b)+2 b,
\end{aligned}
$$

so $f$ satisfies $(* *)$ with $\Omega(n):=\lceil L \cdot(n+b)\rceil+2 b$. Likewise, one obtains that $f$ HölderLipschitz continuous with constants $L, \alpha$ satisfies $(* *)$ with $\Omega(n):=\left\lceil L \cdot(n+b)^{\alpha}\right\rceil+2 b$.

For $f$ uniformly continuous functions with modulus $\omega$ the argument is similar to that in the hyperbolic case: assuming $\|\tilde{z}\| \leq n$ and using $\|z\| \leq b$ we obtain $\|\tilde{z}-z\| \leq n+b$. Dividing the line segment between $\tilde{z}, z$ into $(n+b) \cdot 2^{\omega(0)}+1$ pieces of length $<2^{-\omega(0)}$ we obtain $\|f(\tilde{z})-f(z)\| \leq(n+b) \cdot 2^{\omega(0)}+1$. Thus we obtain

$$
\|f(\tilde{z})\| \leq\|f(\tilde{z})-f(z)\|+\|f(z)-z\|+\|z\| \leq(n+b) \cdot 2^{\omega(0)}+2 b+1,
$$

i.e. $f$ satisfies $(* *)$ with $\Omega(n):=(n+b) \cdot 2^{\omega(0)}+2 b+1$ for uniformly continuous functions $f$ with modulus $\omega$.

As in the metric case, for weakly quasi-nonexpansive functions, the fixed point $p$ is an additional parameter and we require the premise $\|p\| \leq b$. For weakly quasi-nonexpansive functions $f$, we then obtain $\Omega(n):=n+2 b$ as follows:

$\|\tilde{z}\| \leq n \rightarrow\|f(\tilde{z})\| \leq\|f(\tilde{z})-p\|+\|p\| \leq\|\tilde{z}-p\|+\|p\| \leq\|\tilde{z}\|+\|p\|+\|p\| \leq n+2 b$.

At last, if $f$ satisfies $\|z-f(z)\| \leq b$ and $(*)$ with an $\Omega_{0}$, then $f$ satisfies $(* *)$ with $\Omega(n):=\Omega_{0}(n+b)+2 b$.

The result then follows from Theorem 6.3 using as in the proof of Corollary 4.17 the representation of $P, K$.

Remark 6.7. The previous Remarks 4.22, 4.21 apply accordingly in the normed case.

Defining, in the setting of normed linear spaces, the notions of Fix $(f)$ and $F i x_{\varepsilon}(f, z, b)$ as before, we prove the following corollary.

Corollary 6.8. $\quad$ (1) Let $P$ (resp. $K$ ) be a $\mathcal{A}^{\omega}$-definable Polish (resp. compact) metric space and let $B_{\forall}$ and $C_{\exists}$ be as before. If $\mathcal{A}^{\omega}[X,\|\cdot\|, C]_{-b}$ proves a sentence

$\forall x \in P \forall y \in K \forall z^{C}, f^{C \rightarrow C}\left(f\right.$ n.e. $\left.\wedge F i x(f) \neq \emptyset \wedge \forall u^{0} B_{\forall} \rightarrow \exists v^{0} C_{\exists}\right)$, 
then there exists a computable functional $\Phi^{1 \rightarrow 0 \rightarrow 0}$ (on representatives $r_{x}$ : $\mathbb{N} \rightarrow \mathbb{N}$ of elements of $P$ ) s.t. for all $r_{x} \in \mathbb{N}^{\mathbb{N}}, b \in \mathbb{N}$

$$
\begin{aligned}
\forall y \in & K \forall \forall z^{C}, f^{C \rightarrow C}\left(f \text { n.e. } \wedge \forall \varepsilon>0 F i x_{\varepsilon}(f, z, b) \neq \emptyset\right. \\
& \left.\wedge\|z\|_{X},\|z-f(z)\|_{X} \leq_{\mathbb{R}}(b)_{\mathbb{R}} \wedge \forall u^{0} \leq \Phi\left(r_{x}, b\right) B_{\forall} \rightarrow \exists v^{0} \leq \Phi\left(r_{x}, b\right) C_{\exists}\right)
\end{aligned}
$$

holds in all nontrivial (real) normed linear spaces $(X,\|\cdot\|)$ with nonempty convex subset $C$ for which $\left\|c_{X}\right\| \leq b$. $b$.

If $c_{X}$ does not occur in $B_{\forall}, C_{\exists}$, we can drop the requirement that $\left\|c_{X}\right\| \leq$

Analogously, for (real) uniformly convex spaces $(X,\|\cdot\|, C, \eta)$ and (real) inner product spaces $(X,\langle\cdot, \cdot\rangle)$, where for uniformly convex spaces the bound $\Phi$ additionally depends on the modulus of uniform convexity $\eta$.

(2) The corollary also holds if ' $f$ n.e.' is replaced by $f$ Lipschitz continuous, Hölder-Lipschitz continuous or uniformly continuous, where the extracted bound will then depend on the respective constants and moduli.

(3) Considering the premise ' $f$ weakly quasi-nonexpansive', i.e.

$$
\exists p^{C}\left(f(p)=_{X} p \wedge \forall w^{C}\left(\|f(p)-f(w)\|_{X} \leq_{\mathbb{R}}\|p-w\|_{X}\right)\right)
$$

instead of ' $f$ n.e. $\wedge F i x(f) \neq \emptyset$ ', we may weaken this premise to

$$
\begin{aligned}
\forall \varepsilon>0 \exists p^{C}\left(\|f(p)-p\|_{X} \leq_{\mathbb{R}} \varepsilon \wedge\|z-p\|_{X} \leq \mathbb{R}(b)_{\mathbb{R}}\right. \\
\left.\wedge \forall w^{C}\left(\|f(p)-f(w)\|_{X} \leq_{\mathbb{R}}\|p-w\|_{X}\right)\right) .
\end{aligned}
$$

(4) Let $\Psi:(X \rightarrow X) \rightarrow X \rightarrow 1$ be a closed term of $\mathcal{A}^{\omega}[X,\|\cdot\|, C]_{-b}$ (without $\left.c_{X}\right)$; then in (1) and (2) instead of ' $F i x(f) \neq \emptyset$ ' we may weaken $\exists p^{C}\left(\Psi(f, p)=\mathbb{R}_{\mathbb{R}} 0\right)$ ' expressing that $\Psi(f, \cdot)$ has a root $p$ in $C$ to $\forall \varepsilon>$ $0 \exists p^{C}\left(\|z-p\|_{X} \leq_{\mathbb{R}}(b)_{\mathbb{R}} \wedge|\Psi(f, p)| \leq_{\mathbb{R}} \varepsilon\right)^{\prime}$ ', expressing that $\Psi(f, \cdot)$ has $\varepsilon-$ roots $p$ which are $b$-close to $z$ for every $\varepsilon>0$.

Again we may have tuples $\underline{x}, \underline{y}$ of variables ranging over various Polish, resp. compact, metric spaces.

Proof. This essentially is the same proof as for Corollary 4.26, except that $b$ not only bounds the distance between $z$ and $f(z)$ and the diameter of the subset where $\varepsilon$-fixed points are to be found, but also the norm of $z$ itself and the norm of the element $c_{X}$ of the convex subset $C$. The result then follows using Corollary 6.6.

Remark 6.9. Remark 4.27 applies to the above result as well.

Similar to Corollary 5.2 we may also in the setting of normed linear spaces allow a Herbrand normal form version of the previous corollary, which allows one to weaken premises even though the conclusion is of a too general form to allow extraction of explicit bounds.

\section{Simultaneous treatment of several spaces}

The generalized approach to majorization developed in the previous section may also be extended to simultaneously cover finite collections of spaces. Instead of a single space $X$ and a single element $a \in X$ we may have a collection of spaces $X_{1}, \ldots, X_{n}$ and corresponding elements $a_{i} \in X_{i}$ that we take as reference points for the majorization relation. We then may consider elements of products of these spaces and functions between such product-elements. 
$\mathbf{T}^{X_{1}, \ldots, X_{n}}$ is the set of all finite types $\rho$ over the ground types $0, X_{1}, \ldots, X_{n}$. For $\rho \in \mathbf{T}^{X_{1}, \ldots, X_{n}}$ the type $\hat{\rho}$ defines the type which results from $\rho$ by replacing all occurrences of $X_{i}, 1 \leq i \leq n$, by 0 . The relation $\gtrsim \underline{a}$ is then defined as follows:

Definition 7.1. We define a ternary relation $\gtrsim \frac{a}{\rho}$ between objects $x, y$ and an $n$ tuple $\underline{a}$ of type $\widehat{\rho}, \rho$ and $X_{1}, \ldots, X_{n}$, respectively, as follows:

- $x^{0} \geq \frac{a}{0} y^{0}: \equiv x \geq_{\mathbb{N}} y$,

- $x^{0} \gtrsim \frac{a}{X_{i}} y^{X_{i}}: \equiv(x)_{\mathbb{R}} \geq_{\mathbb{R}} d_{X_{i}}\left(y, a_{i}\right)$,

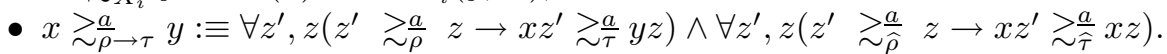

If $X_{i}$ is a normed linear space we require $a_{i}=0_{X_{i}}$ s.t. $d_{X_{i}}\left(x, a_{i}\right)={ }_{\mathbb{R}}\|x\|_{X_{i}}$.

E.g. if we have two metric spaces $\left(X_{1}, d_{X_{1}}\right)$ and $\left(X_{2}, d_{X_{2}}\right)$, then an $\left(a_{1}, a_{2}\right)$ majorant for $f^{X_{1} \rightarrow X_{2}}$ is a monotone function $f^{*}$ of type 1 such that

$$
\forall n^{0}, x^{X_{1}}\left(d_{X_{1}}\left(x, a_{1}\right) \leq_{\mathbb{R}}(n)_{\mathbb{R}} \rightarrow d_{X_{2}}\left(f(x), a_{2}\right) \leq_{\mathbb{R}}\left(f^{*}(n)\right)_{\mathbb{R}}\right) .
$$

If $f$ is nonexpansive and $a_{2}:=f\left(a_{1}\right)$, then $f$ is $\left(a_{1}, a_{2}\right)$-majorized by the identity function $\lambda n . n^{0}$.

Functions involving product types are treated using "currying" in the form of the following two patterns:

- a function $f: X_{1} \times \ldots \times X_{n} \rightarrow \rho$ is represented by $f: X_{1} \rightarrow \ldots \rightarrow X_{n} \rightarrow \rho$,

- a function $\rho \rightarrow X_{1} \times \ldots \times X_{n}$ is represented by an $n$-tuple of functions $f_{i}: \rho \rightarrow X_{i}$.

Thus e.g. a function $f: X_{1} \times X_{2} \rightarrow X_{1} \times X_{2}$ will be represented by a pair $f_{1,2}: X_{1} \rightarrow\left(X_{2} \rightarrow X_{1,2}\right)$, a function $g:\left(X_{1} \times X_{2} \rightarrow X_{1} \times X_{2}\right) \rightarrow X_{1} \times X_{2}$ by a pair $g_{1,2}:\left(X_{1} \rightarrow\left(X_{2} \rightarrow X_{1}\right)\right) \rightarrow\left(\left(X_{1} \rightarrow\left(X_{2} \rightarrow X_{2}\right)\right) \rightarrow X_{1,2}\right)$ and similarly for products of greater arity and functions of more complex types.

\section{Applications}

Application 8.1. Let $(X, d, W)$ be an arbitrary (nonempty) hyperbolic space, $k \in \mathbb{N}, k \geq 1$, and $\left(\lambda_{n}\right)_{n \in \mathbb{N}}$ a sequence in $\left[0,1-\frac{1}{k}\right]$ with $\sum_{n=0}^{\infty} \lambda_{n}=\infty$, and define for $f: X \rightarrow X, x \in X$ the Krasnoselski-Mann iteration $\left(x_{n}\right)_{n}$ starting from $x([30,35])$ by

$$
x_{0}:=x, x_{n+1}:=\left(1-\lambda_{n}\right) x_{n} \oplus \lambda_{n} f\left(x_{n}\right) .
$$

In [11] (Theorem 1) the following is proved $^{21}$

$$
\forall x \in X, f: X \rightarrow X\left(\left(x_{n}\right)_{n} \text { bounded and } f \text { n.e. } \rightarrow \lim _{n \rightarrow \infty} d\left(x_{n}, f\left(x_{n}\right)\right)=0\right) .
$$

As observed in [2], it actually suffices to assume that $\left(x_{n}^{*}\right)_{n}$ starting from some $x^{*}$ is bounded. Therefore

$\forall x \in X, f: X \rightarrow X\left(\exists x^{*} \in X\left(\left(x_{n}^{*}\right)_{n}\right.\right.$ bounded $)$ and $f$ n.e. $\left.\rightarrow \lim _{n \rightarrow \infty} d\left(x_{n}, f\left(x_{n}\right)\right)=0\right)$.

The proof given in [11] (and [2]) can easily be formalized in $\mathcal{A}^{\omega}[X, d, W]_{-b}$ (see [25] for more details on this). As an application of Corollary 4.20 we obtain (see the proof below) the following effective and uniform version: There exists a computable bound $\Phi(k, \alpha, b, l)$ such that in any (nonempty) hyperbolic space $(X, d, W)$, for any

\footnotetext{
${ }^{21}$ For the case of convex subsets $C \subseteq X$ of normed linear spaces $(X,\|\cdot\|)$ this result is already due to [13]. [11] even treats spaces of hyperbolic type.
} 
$l, b, k \in \mathbb{N}$ and any $\alpha: \mathbb{N} \rightarrow \mathbb{N}$ the following holds: if $\left(\lambda_{n}\right)$ is a sequence in $[0,1)$ such that

then

$$
\forall n \in \mathbb{N}\left(\lambda_{n} \leq 1-\frac{1}{k} \wedge n \leq \sum_{i=0}^{\alpha(n)} \lambda_{i}\right),
$$

$$
\begin{aligned}
& \forall x, x^{*} \in X \forall f: X \rightarrow X \\
& \quad\left(\forall i, j\left(d\left(x, x^{*}\right), d\left(x_{i}^{*}, x_{j}^{*}\right) \leq b\right) \wedge f \text { n.e. } \rightarrow \forall m \geq \Phi(k, \alpha, b, l) d\left(x_{m}, f\left(x_{m}\right)\right)<2^{-l}\right) .
\end{aligned}
$$

Proof. As mentioned already, $\mathcal{A}^{\omega}[X, d, W]_{-b}$ proves the following (formalized version of Theorem 1 in [11]): if $k \geq 1, \lambda_{(\cdot)}^{0 \rightarrow 1}$ represents an element of the compact Polish space $[0,1]^{\infty}$ (with the product metric) and $\alpha: \mathbb{N} \rightarrow \mathbb{N}$ such that

$$
\forall n \in \mathbb{N}\left(\lambda_{n} \leq_{\mathbb{R}} 1-\frac{1}{k} \wedge n \leq_{\mathbb{R}} \sum_{i=0}^{\alpha(n)} \lambda_{i}\right),
$$

where $\sum_{i=0}^{\alpha(n)} \lambda_{i}$ represents the corresponding summation of the real numbers in $[0,1]$ represented by $\lambda_{i}$, then

$\forall l, b \in \mathbb{N}, x, x^{*}, f^{X \rightarrow X}\left(\left(x_{n}^{*}\right)_{n} b\right.$-bounded $\wedge f$ n.e. $\left.\rightarrow \exists n \in \mathbb{N}\left(d_{X}\left(x_{n}, f\left(x_{n}\right)\right)<\mathbb{R}^{-l}\right)\right)$, where ' $(*)$ ' and ' $\left(x_{n}^{*}\right)_{n} b$-bounded' are $\forall$-formulas and ' $d_{X}\left(x_{n}, f\left(x_{n}\right)\right)<\mathbb{R} 2^{-l}$ ' is an $\exists$-formula.

Now Corollary 4.20 yields the existence of a computable functional $\Phi(k, \alpha, b, l)$ such that for $n:=\Phi(k, \alpha, b, l)$

$$
\begin{array}{r}
\forall\left(\lambda_{m}\right) \in[0,1]^{\infty} \forall x, x^{*} \in X \forall f: X \rightarrow X\left((*) \wedge \forall i, j\left(d(x, f(x)), d\left(x, x^{*}\right), d\left(x_{i}^{*}, x_{j}^{*}\right) \leq b\right)\right. \\
\left.\wedge f \text { n.e. } \rightarrow \exists m \leq n\left(d\left(x_{m}, f\left(x_{m}\right)\right)<2^{-l}\right)\right)
\end{array}
$$

holds for all $k, \alpha, b, l$ in any (nonempty) hyperbolic space $(X, d, W)$.

Since $\left(d\left(x_{n}, f\left(x_{n}\right)\right)\right)_{n}$ is a nonincreasing sequence $([11])$ the conclusion actually implies

$$
\forall m \geq \Phi(k, \alpha, b, l)\left(d\left(x_{m}, f\left(x_{m}\right)\right)<2^{-l}\right) .
$$

The only thing which remains to show is that the assumption ' $d(x, f(x)) \leq b$ ' is redundant: by Theorem 1 from [11] we know, in particular, that $d\left(x_{n}^{*}, f\left(x_{n}^{*}\right)\right) \rightarrow 0$ and so a fortiori

$$
\exists n \in \mathbb{N}\left(d\left(x_{n}^{*}, f\left(x_{n}^{*}\right)\right) \leq b\right) .
$$

Using $d\left(x, x^{*}\right), d\left(x_{i}^{*}, x_{j}^{*}\right) \leq b$ for all $i, j$ and the nonexpansivity of $f$ yields

$$
\begin{aligned}
d(x, f(x)) \leq & d\left(x, x^{*}\right)+d\left(x^{*}, x_{n}^{*}\right)+d\left(x_{n}^{*}, f\left(x_{n}^{*}\right)\right)+d\left(f\left(x_{n}^{*}\right), f\left(x^{*}\right)\right) \\
& +d\left(f\left(x^{*}\right), f(x)\right) \leq 5 b .
\end{aligned}
$$

So replacing ' $b$ ' in the bound by ' $5 b$ ' we can drop the assumption ' $d(x, f(x)) \leq$ $b^{\prime}$.

As a corollary it follows that for bounded hyperbolic spaces $(X, d, W)$ the convergence $d\left(x_{n}, f\left(x_{n}\right)\right) \rightarrow 0$ is uniform in $x, f$ and - except for a bound $b$ on the metric - in $(X, d, W)$. This corollary was first proved as Theorem 2 in $[11]^{22}$ and was shown to follow from a general logical metatheorem in [25] where a detailed

\footnotetext{
${ }^{22}$ For the case of bounded convex subsets of normed spaces and constant $\lambda_{n}=\lambda \in(0,1)$ the uniformity in $x$ was already shown in [7] and - for $\left(\lambda_{n}\right)_{n}$ in $[a, b] \subset(0,1)$ and non-increasing - in $[5]$.
} 
discussion of this point is given. In [28], the extraction of an actual effective uniform rate of convergence was carried out and it was noticed that the assumption on $X$ to be bounded could be weakened to a bound $b$ on $d\left(x, x^{*}\right)$ and $\left(x_{n}^{*}\right)_{n}$ for some $x^{*} \in X$. At that time, there was no explanation in terms of a general result from logic for the fact that these local bounds where sufficient. This latter fact can now for the first time be explained by our refined logical metatheorems as well. Note that the proof of Theorem 2 in [11] (as well as the alternative proof for constant $\lambda_{n}=\lambda \in(0,1)$ given in [15]) crucially uses that the whole space $X$ is assumed to be bounded. So the uniformity result guaranteed a priorily by the metatheorems of the present paper applied to Theorem 1 of [11] not only immediately yields Theorem 2 from [11] (called the 'main result') but even a qualitatively stronger uniformity which apparently cannot be obtained by the functional analytic embedding techniques used in [11] (or in [15]).

The aforementioned explicit bound extracted in [28] (see theorem 3.21 and remark 3.13) is as follows (for the case of convex subsets of normed linear spaces the result is already due to $[21,23])$ :

$$
\begin{aligned}
& \Phi(k, \alpha, b, l):=\widehat{\alpha}(\lceil 12 b \cdot \exp (k(M+1))\rceil-1, M), \text { where } \\
& M:=(1+6 b) \cdot 2^{l}, \widehat{\alpha}(0, n):=\tilde{\alpha}(0, n), \widehat{\alpha}(i+1, n):=\tilde{\alpha}(\widehat{\alpha}(i, n), n), \text { with } \\
& \tilde{\alpha}(i, n):=i+\alpha^{+}(i, n), \text { where } \alpha^{+}(i, n):=\max _{j \leq i}[\alpha(n+j)-j+1] .
\end{aligned}
$$

Before we come to the next application we need the following

Proposition $8.2([13,11])$. Let $(X, d, W)$ be a (nonempty) compact hyperbolic space and $\left(\lambda_{n}\right), f,\left(x_{n}\right)$ as in Application 8.1. Then $\left(x_{n}\right)_{n}$ converges towards a fixed point of $f$ (see [24] for details).

Proof. By the result mentioned in Application 8.1 we have that $d\left(x_{n}, f\left(x_{n}\right)\right) \rightarrow 0$ since the compactness of $X$ implies that $X$ - and hence $\left(x_{n}\right)_{n}$ - is bounded. Again using the compactness of $X$, we know that $\left(x_{n}\right)_{n}$ has a convergent subsequence $\left(x_{n_{k}}\right)_{k}$ with limit $\widehat{x}$. One easily shows (using the continuity of $f$ ) that $\widehat{x}$ is a fixed point of $f$. The proof is concluded by verifying the easy fact that for any fixed point $\widehat{x}$ of $f$

$$
\forall n \in \mathbb{N}\left(d\left(x_{n+1}, \widehat{x}\right) \leq d\left(x_{n}, \widehat{x}\right)\right),
$$

which implies that $\left(x_{n}\right)_{n}$ already converges towards $\widehat{x}$.

In particular it follows that $\left(x_{n}\right)_{n}$ is a Cauchy sequence, and for this corollary one does not need the completeness of $X$ but only its total boundedness: suppose $X$ is totally bounded. Then its metric completion $\widehat{X}$ (which again is a hyperbolic space) is totally bounded too and hence compact. $f$ extends to a nonexpansive function $\widehat{f}$ on the completion so that the previous result applies. Since $\widehat{f}$ coincides with $f$ on $X$, the Krasnoselski-Mann iteration of $\widehat{f}$ also coincides with that of $f$ when starting from a point $x \in X$. Hence we conclude that $\left(x_{n}\right)_{n}$ is a Cauchy sequence.

Application 8.3. Let us consider the proof of the Cauchy property of $\left(x_{n}\right)$ from the asymptotic regularity (i.e. $d\left(x_{n}, f\left(x_{n}\right)\right) \rightarrow 0$ ) (taken as an assumption) $)^{23}$ under

\footnotetext{
${ }^{23}$ The proof relative to this assumption only uses the fact that $\left(\lambda_{n}\right)_{n}$ is a sequence in $[0,1]$ but not the other assumptions on $\left(\lambda_{n}\right)_{n}$ (which are only needed to prove that $d\left(x_{n}, f\left(x_{n}\right)\right) \rightarrow 0$ ).
} 
the additional assumption of $X$ being totally bounded, i.e. the proof of

$(+) \quad X$ totally bounded $\wedge \lim d\left(x_{n}, f\left(x_{n}\right)\right)=0 \wedge f$ n.e.

$$
\rightarrow \forall k \in \mathbb{N} \exists n \in \mathbb{N} \forall m \geq n\left(d\left(x_{n}, x_{m}\right) \leq 2^{-k}\right)
$$

which can be formalized in $\mathcal{A}^{\omega}[X, d, W]_{-b}$.

In order to apply Corollary 4.20 we first have to modify $(+)$ so that the logical form required in the corollary is obtained. In order to do so we first have to make the assumptions explicit:

- due to the fact that $d\left(x_{n}, f\left(x_{n}\right)\right)_{n}$ is nonincreasing, we can write the asymptotic regularity equivalently as $\forall l \in \mathbb{N} \exists n \in \mathbb{N}\left(d\left(x_{n}, f\left(x_{n}\right)\right) \leq 2^{-l}\right)$ which asks for a witnessing rate of asymptotic regularity $\delta: \mathbb{N} \rightarrow \mathbb{N}$ such that

$$
\forall l \in \mathbb{N}\left(d\left(x_{\delta(l)}, f\left(x_{\delta(l)}\right)\right) \leq 2^{-l}\right),
$$

- the total boundedness of $X$ is expressed by the existence of a sequence $\left(a_{n}\right)_{n}$ of points in $X$ and a function $\gamma: \mathbb{N} \rightarrow \mathbb{N}$ such that

$$
\forall l \in \mathbb{N}, x \in X \exists n \leq \gamma(l)\left(d\left(x, a_{n}\right) \leq 2^{-l}\right) .
$$

A function $\gamma$ such that a sequence $\left(a_{n}\right)_{n}$ in $X$ satisfying (2) exists is called a modulus of total boundedness for $X$.

It is important to notice that both (1) and (2) are (provably equivalent to) $\forall$-formulas.

The conclusion, i.e. the Cauchy property of $\left(x_{n}\right)$, is a $\Pi_{3}^{0}$-formula and so too complicated to be covered by our metatheorems. In fact, as shown in [24] there is no Cauchy rate computable in the parameters even for a very simple computable sequence of nonexpansive functions on $X=[0,1]$ and $\lambda_{n}=\frac{1}{2}$. We therefore modify the conclusion to its Herbrand normal form ${ }^{24}$

$$
\forall l \in \mathbb{N} \forall g: \mathbb{N} \rightarrow \mathbb{N} \exists n \in \mathbb{N} \forall i, j \in[n ; n+g(n)]\left(d\left(x_{i}, x_{j}\right)<2^{-l}\right),
$$

where $[n ; m]$ denotes the subset $\{n, n+1, \ldots, m-1, m\}$ of $\mathbb{N}$ for $m \geq n$.

Classically, $(H)$ is equivalent to the Cauchy property for $\left(x_{n}\right)_{n}$ but - since the proof is ineffective - a computable bound on $(H)$ does not yield a computable Cauchy modulus for $\left(x_{n}\right)_{n}$. Note that

$$
\exists n \in \mathbb{N} \forall i, j \in[n ; n+g(n)]\left(d\left(x_{i}, x_{j}\right)<2^{-l}\right)
$$

is (equivalent to) an $\exists$-formula.

$\mathcal{A}^{\omega}[X, d, W]_{-b}$ proves that

$$
\begin{aligned}
& \forall\left(\lambda_{m}\right) \in[0,1]^{\infty} \forall x^{X} \forall f^{X \rightarrow X},\left(a_{n}\right)^{0 \rightarrow X}, l^{0}, \gamma^{1}, \delta^{1}, g^{1} \\
& \quad\left((1) \wedge(2) \wedge f \text { n.e. } \rightarrow \exists m \in \mathbb{N} \forall i, j \in[m ; m+g(m)]\left(d\left(x_{i}, x_{j}\right)<2^{-l}\right)\right) .
\end{aligned}
$$

The total boundedness of $X$ implies that the metric of $X$ is bounded and a bound can be computed by $b:=\max \left\{d\left(a_{i}, a_{j}\right): i, j \leq \gamma(0)\right\}+2$. However, in order to guarantee our result to be independent from $\left(a_{n}\right)_{n}$ we add a bound $b$ of $X$ as an additional input. Hence by Corollary 4.15 we obtain a computable bound $\Omega(l, b, \gamma, \delta, g)$ such that for all $\left(\lambda_{n}\right)$ in $[0,1], x \in X,\left(a_{n}\right)$ in $X, f: X \rightarrow X, l \in \mathbb{N}$ and $\gamma, \delta, g: \mathbb{N} \rightarrow \mathbb{N}:$

$$
(1) \wedge(2) \wedge f \text { n.e. } \rightarrow \exists m \leq \Omega(l, b, \gamma, \delta, g) \forall i, j \in[m ; m+g(m)]\left(d\left(x_{i}, x_{j}\right)<2^{-l}\right)
$$

\footnotetext{
${ }^{24}$ More precisely, the Herbrand normal form of a slightly different but trivially equivalent formulation of the Cauchy property.
} 
holds in any (nonempty) $b$-bounded, totally bounded (with modulus $\gamma$ ) hyperbolic space $(X, d, W)$.

A concrete bound $\Omega$ of this kind has in fact been extracted first in [24], where the extraction itself was guided by the algorithm provided by the proof of Corollary 4.15 as well as the proof-theoretic study of the Bolzano-Weierstraß principle carried out in [20]. This concrete $\Omega$ is even independent from $b$ and is defined as follows:

$$
\Omega(l, g, \delta, \gamma):=\max _{i \leq \gamma(l+3)} \Psi_{0}(i, l, g, \delta),
$$

where

$$
\left\{\begin{array}{l}
\Psi_{0}(0, l, g, \delta):=0 \\
\Psi_{0}(n+1, l, g, \delta):=\delta\left(l+2+\left\lceil\log _{2}\left(\max _{i \leq n} g\left(\Psi_{0}(i, l, g, \delta)\right)+1\right)\right\rceil\right) .
\end{array}\right.
$$

For $X$ being $b$-bounded and $\left(\lambda_{n}\right)$ s.t. $\lambda_{i} \in\left[0,1-\frac{1}{k}\right], \sum_{i=0}^{\alpha(n)} \lambda_{i} \geq n$, we can take $\delta(l):=\Phi(k, \alpha, b, l)$ from Application 8.1.

Application 8.4. Let $(X, d, W), k,\left(\lambda_{n}\right), f, x$ and $\left(x_{n}\right)$ be as in Application 8.1. In [2], the following result is proved:

$$
\forall x \in X, f: X \rightarrow X\left(f \text { n.e. } \rightarrow \lim _{n \rightarrow \infty} d\left(x_{n}, f\left(x_{n}\right)\right)=r(f)\right),
$$

where $r(f):=\inf _{y \in X} d(y, f(y))$ is the so-called minimal displacement of $f$. As $\left(x_{n}\right)$ is no longer assumed to be bounded, $r(f)$ can very well be strictly positive: e.g. for $\mathbb{R}$ (with the natural metric) and $f: \mathbb{R} \rightarrow \mathbb{R}, f(x):=x+1$ we have $r(f)=1$ although $f$ is nonexpansive.

The above theorem can be written equivalently as follows (again using the fact that $\left(d\left(x_{n}, f\left(x_{n}\right)\right)\right)$ is nonincreasing $):^{25}$

$$
\forall l \in \mathbb{N} \forall x, x^{*} \in X, f: X \rightarrow X \exists n \in \mathbb{N}\left(d\left(x_{n}, f\left(x_{n}\right)\right)<d\left(x^{*}, f\left(x^{*}\right)\right)+2^{-l}\right) .
$$

The proof given in [2] can be formalized in $\mathcal{A}^{\omega}[X, d, W]_{-b}$, and so Corollary 4.20 yields (like in the proof of Application 8.1 above) an effective bound $n:=\Psi(k, \alpha, b, l)$ such that in any (nonempty) hyperbolic space $(X, d, W)$, for any $l, b, k \in \mathbb{N}$ and any $\alpha: \mathbb{N} \rightarrow \mathbb{N}$ such that $\lambda_{n} \leq 1-\frac{1}{k}$ and $n \leq \sum_{i=0}^{\alpha(n)} \lambda_{i}$ the following holds:

$$
\begin{aligned}
& \forall x, x^{*} \in X \forall f: X \rightarrow X \\
& \quad\left(d\left(x, x^{*}\right), d(x, f(x)) \leq b \wedge f \text { n.e. } \rightarrow \exists k \leq n\left(d\left(x_{k}, f\left(x_{k}\right)\right)<d\left(x^{*}, f\left(x^{*}\right)\right)+2^{-l}\right)\right) .
\end{aligned}
$$

Therefore (by the fact that $\left(d\left(x_{n}, f\left(x_{n}\right)\right)\right)_{n}$ is nonincreasing)

$$
\begin{aligned}
\forall x, x^{*} \in X \forall f & : X \rightarrow X\left(d\left(x, x^{*}\right), d(x, f(x)) \leq b \wedge f\right. \text { n.e. } \\
& \left.\rightarrow \forall m \geq \Psi(k, \alpha, b, l)\left(d\left(x_{m}, f\left(x_{m}\right)\right)<d\left(x^{*}, f\left(x^{*}\right)\right)+2^{-l}\right)\right) .
\end{aligned}
$$

Such an explicit bound $\Psi$ (which is very similar to the bound $\Phi$ mentioned in connection with Application 8.1) has been extracted first in [28] (for the special case of convex subsets of normed spaces this is already due to [21] and - in a stronger form - in [23]). Our refined metatheorems for the first time allow one to explain this finding as an instance of a general result in logic. For functional analytic applications of the uniformity provided by $\Psi$ see [27].

\footnotetext{
${ }^{25}$ For details see [23].
} 


\section{Proofs of Theorems 4.10 And 6.3}

We focus on proving Theorems 4.10 and 6.3 for the theories $\mathcal{A}^{\omega}[X, d, W]_{-b}$ and $\mathcal{A}^{\omega}[X,\|\cdot\|, C]_{-b}$, respectively. From these proofs, the corresponding proofs for the other variants of the theories for metric and normed linear spaces can easily be obtained by treating one of the following kinds of extensions: (1) adding another purely universal axiom to the theory (purely universal axioms are their own functional interpretation), e.g. for $\mathcal{A}^{\omega}[X, d, W, \mathrm{CAT}(0)]_{-}$, or (2) adding a new majorizable constant to the language as e.g. for $\mathcal{A}^{\omega}[X,\|\cdot\|, C, \eta]_{-b}$, where the modulus of uniform convexity $\eta$ is given as a number theoretic function $\eta: \mathbb{N} \rightarrow \mathbb{N}$.

The proofs of Theorems 4.10 and 6.3 closely follow the general proof outline in [25], but both the interpretation of the theories $\mathcal{A}^{\omega}[X, d, W]_{-b}, \mathcal{A}^{\omega}[X,\|\cdot\|, C]_{-b}$ by bar-recursive functionals and the subsequent interpretation of these functionals in an extension of the Howard-Bezem strongly majorizable functionals to all types $\mathbf{T}^{X}$ are now parametrized by an element $a \in X$ for the relation $\gtrsim^{a}$. For the interpretation of $\mathcal{A}^{\omega}[X, d, W]_{-b}$ by bar-recursive functionals this in effect leads to a family of functional interpretations parametrized by $a \in X$, where the interpretation of the element $a \in X$ is fixed later during the majorization process (see Remark 9.10). For $\mathcal{A}^{\omega}[X,\|\cdot\|, C]_{-b}$ the choice $a=0_{X}$ is fixed from the beginning.

Based on $\gtrsim^{a}$ we redefine Kohlenbach's [25] extension of Bezem's [1] type structure of hereditarily strongly majorizable set-theoretical functionals to all types $\mathbf{T}^{X}$ (based on $\gtrsim^{a}$ instead of s-maj) as follows:

Definition 9.1. Let $(X, d)$ be a nonempty metric space, resp. $(X,\|\cdot\|)$ a nontrivial real normed linear space, and let $a \in X$ be given. The extensional type structure $\mathcal{M}^{\omega, X}$ of all hereditarily strongly $a$-majorizable set-theoretic functionals of type $\rho \in \mathbf{T}^{X}$ over $\mathbb{N}$ and $X$ is defined as

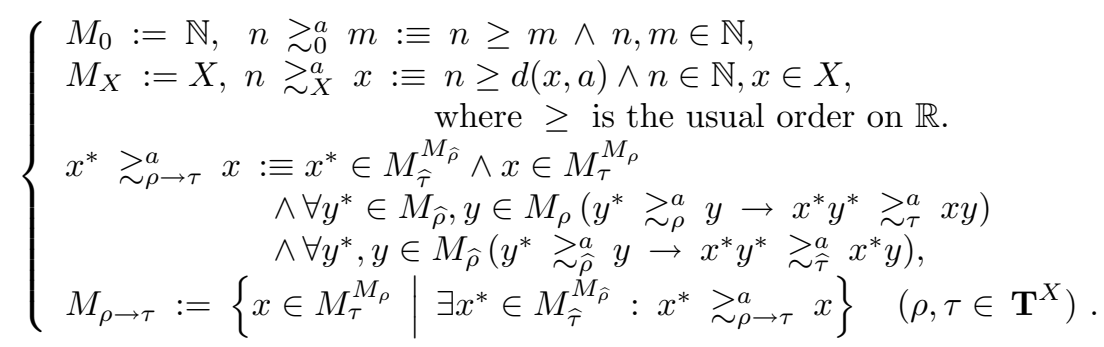

Remark 9.2. Restricted to the types $\mathbf{T}$, this type structure is identical to Bezem's original type structure $\mathcal{M}^{\omega}$ of strongly hereditarily majorizable functionals. This is because for $\rho \in \mathbf{T}$ the relations s-maj $\mathrm{j}_{\rho}$ and $\gtrsim_{\rho}^{a}$ are the same and hence for $\rho \in \mathbf{T}$ we may freely write s-maj $j_{\rho}$ instead of $\gtrsim_{\rho}^{a}$, as here the parameter $a \in X$ is irrelevant.

Even though the $a$-majorization relation is parametrized by an element $a \in X$, the resulting model of all hereditarily strongly $a$-majorizable functionals is independent of the choice of $a \in X$, as the following lemma shows:

Lemma 9.3. For every $\rho \in \mathbf{T}^{X}$ there is a (primitive recursive) mapping $\Phi_{\rho}$ in $M_{\widehat{\rho} \rightarrow 0 \rightarrow \widehat{\rho}}$ s.t. for any given $a, b \in X$, all $x^{*} \in M_{\widehat{\rho}}, x \in M_{\rho}^{a}$ and all $n \in \mathbb{N}$ s.t. $d(a, b) \leq n$,

$$
x^{*} \gtrsim_{\rho}^{a} x \rightarrow \Phi_{\rho}\left(x^{*}, n\right) \gtrsim_{\rho}^{b} x,
$$

and for all $x^{*}, \widehat{x} \in M_{\widehat{\rho}}, n, m \in \mathbb{N}$

$$
x^{*} s-m a j_{\widehat{\rho}} \widehat{x} \wedge n \geq m \rightarrow \Phi_{\rho}\left(x^{*}, n\right) s-m a j_{\widehat{\rho}} \Phi_{\rho}(\widehat{x}, m) .
$$


In particular: $M_{\rho}^{a}=M_{\rho}^{b}$ and - trivially $-M_{\widehat{\rho}}^{a}=M_{\widehat{\rho}}^{b}$. Note, that this property is symmetric in $a, b \in X$.

Proof. The proof is by induction on the type $\rho \in \mathbf{T}^{X}$.

For $\rho=0$ define $\Phi_{0}(x, n):=x$. Trivially $M_{0}^{a}=M_{0}^{b}$ by definition.

For $\rho=X$ the mapping $\Phi_{X}$ is the mapping $\Phi_{X}\left(x^{*}, n\right)=x^{*}+n$, as $x^{*} \gtrsim_{X}^{a} x$ is equivalent to $x^{*} \geq d(x, a)$, but then by the triangle inequality $x^{*}+n \geq d(x, b)$ and hence $x^{*}+n \gtrsim_{X}^{b} x$. Obviously, $M_{X}^{a}=M_{X}^{b}$.

For $\rho=\sigma \rightarrow \tau$, we need to construct the mapping $\Phi_{\sigma \rightarrow \tau}$ and show that $x \in$ $M_{\sigma \rightarrow \tau}^{a}$ implies $x \in M_{\sigma \rightarrow \tau}^{b}$. Assume $x^{*} \gtrsim_{\sigma \rightarrow \tau}^{a} x$ for $x \in M_{\sigma \rightarrow \tau}^{a}$, and let $y^{*} \in M_{\widehat{\sigma}}$ and $y \in M_{\sigma}$ be given such that $y^{*} \gtrsim_{\sigma}^{b} y$. By the induction hypothesis for $\sigma$ there is a $\Phi_{\sigma}$ such that, using the symmetry in $a$ and $b, \Phi_{\sigma}\left(y^{*}, n\right) \gtrsim_{\sigma}^{a} y$. Next, by the definition of $\gtrsim_{\sigma \rightarrow \tau}^{a}$ we have that $x^{*}\left(\Phi_{\sigma}\left(y^{*}, n\right)\right) \gtrsim_{\tau}^{a} x y$. But then by the induction hypothesis for $\tau$ there is a mapping $\Phi_{\tau}$ such that

$$
\Phi_{\tau}\left(x^{*}\left(\Phi_{\sigma}\left(y^{*}, n\right)\right), n\right) \gtrsim_{\sigma}^{b} x y .
$$

Also for $y^{*} \mathrm{~s}-m a j_{\widehat{\sigma}} \widehat{y}$ and $n \geq m$ we have by I.H. that $\Phi_{\sigma}\left(y^{*}, n\right) \mathrm{s}-\operatorname{maj}_{\widehat{\sigma}} \Phi_{\sigma}(\widehat{y}, m)$ and so for $x^{*} \mathrm{~s}^{-m a j} \hat{j}_{\hat{\rho}} \widehat{x}$ we get $x^{*}\left(\Phi_{\sigma}\left(y^{*}, n\right)\right) \operatorname{s} \operatorname{maj}_{\widehat{\tau}} \widehat{x}\left(\Phi_{\sigma}(\widehat{y}, m)\right)$ which in turn implies that $\Phi_{\tau}\left(x^{*}\left(\Phi_{\sigma}\left(y^{*}, n\right)\right), n\right)$ s-maj ${ }_{\widehat{\tau}} \Phi_{\tau}\left(\widehat{x}\left(\Phi_{\sigma}(\widehat{y}, m)\right), m\right)$. The desired $\Phi_{\sigma \rightarrow \tau}$ is then obtained by $\lambda$-abstracting $x^{*}, n$ and $y^{*}$. In particular, $\lambda y^{*} . \Phi_{\tau}\left(x^{*} \Phi_{\sigma}\left(y^{*}, n\right), n\right)$ is a $b$-majorant for $x$ and hence $x \in M_{\sigma \rightarrow \tau}^{b}$.

Remark 9.4. Even though it is independent of the choice of $a \in X$ whether or not a certain functional is $a$-majorizable, the complexity and possible uniformities of the majorants depend crucially on the choice of $a \in X$. In particular, for normed linear spaces $a$-majorants for $a \neq 0_{X}$ will usually depend on an upper bound $n \geq\|a\|$ and hence will not have the uniformity w.r.t. $a$ that we aim for in our applications.

We also need the following lemmas:

Lemma 9.5. $x^{*} \gtrsim_{\rho}^{a} x \rightarrow x^{*} \gtrsim_{\widehat{\rho}}^{a} x^{*} \in M_{\widehat{\rho}}$ for all $\rho \in \mathbf{T}^{X}$.

Proof. By induction on $\rho$ using the fact that by definition of $\gtrsim^{a}$ if $x^{*} \gtrsim_{\rho \rightarrow \tau}^{a} x$, then $\forall z^{*}, z\left(z^{*} \gtrsim \widehat{\rho} z \rightarrow x^{*} z^{*} \gtrsim_{\widetilde{\tau}}^{a} x^{*} z\right)$.

Lemma 9.6. Let $\rho=\rho_{1} \rightarrow \ldots \rightarrow \rho_{k} \rightarrow \tau$. Let $x^{*}: M_{\widehat{\rho}_{1}} \rightarrow\left(M_{\widehat{\rho}_{2}} \rightarrow \ldots \rightarrow M_{\widehat{\tau}}\right)$ and $x: M_{\rho_{1}} \rightarrow\left(M_{\rho_{2}} \rightarrow \ldots \rightarrow M_{\tau}\right)$. Then $x^{*} \gtrsim_{\rho}^{a} x$ iff

$$
\begin{aligned}
& \text { (I) } \forall y_{1}^{*}, y_{1}, \ldots, y_{k}^{*}, y_{k}\left(\bigwedge_{i=1}^{k}\left(y_{i}^{*} \gtrsim_{\rho_{i}}^{a} y_{i}\right) \rightarrow x^{*} y_{1}^{*} \ldots y_{k}^{*} \gtrsim_{\tau}^{a} x y_{1} \ldots y_{k}\right) \text { and } \\
& \text { (II) } \forall y_{1}^{*}, y_{1}, \ldots, y_{k}^{*}, y_{k}\left(\bigwedge_{i=1}^{k}\left(y_{i}^{*} \gtrsim_{\rho_{i}}^{a} y_{i}\right) \rightarrow x^{*} y_{1}^{*} \ldots y_{k}^{*} \gtrsim_{\widehat{\tau}}^{a} x^{*} y_{1} \ldots y_{k}\right) .
\end{aligned}
$$

Proof. By induction on $k$. The case $k=1$ follows from the definition of $\gtrsim^{a}$.

$k=n+1$ : Let $\tau_{0}=\rho_{n+1} \rightarrow \tau$. For ' $\Rightarrow$ ', we have by induction hypothesis

$$
\forall y_{1}^{*}, y_{1}, \ldots, y_{n}^{*}, y_{n}\left(\bigwedge_{i=1}^{n}\left(y_{i}^{*} \gtrsim_{\rho_{i}}^{a} y_{i}\right) \rightarrow x^{*} y_{1}^{*} \ldots y_{n}^{*} \gtrsim_{\tau_{0}}^{a} x y_{1} \ldots y_{n}\right) .
$$

Now assume $y_{n+1}^{*} \gtrsim_{\rho_{n+1}}^{a} y_{n+1}$. Then by definition of $\gtrsim^{a}$

$$
x^{*} y_{n}^{*} \ldots y_{n}^{*} y_{n+1}^{*} \gtrsim_{\tau}^{a} x y_{1} \ldots y_{n} y_{n+1},
$$

so (I) follows. (II) can be treated analogously. 
For ' $\Leftarrow$ ', assume

$$
\forall y_{1}^{*}, y_{1}, \ldots, y_{n+1}^{*}, y_{n+1}\left(\bigwedge_{i=1}^{n+1}\left(y_{i}^{*} \gtrsim_{\rho_{i}}^{a} y_{i}\right) \rightarrow x^{*} y_{1}^{*} \ldots y_{n+1}^{*} \gtrsim_{\tau}^{a} x y_{1} \ldots y_{n+1}\right)
$$

and

$$
\forall y_{1}^{*}, y_{1}, \ldots, y_{n+1}^{*}, y_{n+1}\left(\bigwedge_{i=1}^{n+1}\left(y_{i}^{*} \gtrsim \frac{a}{\rho_{i}} y_{i}\right) \rightarrow x^{*} y_{1}^{*} \ldots y_{n+1}^{*} \gtrsim \underset{\tau}{a} x^{*} y_{1} \ldots y_{n+1}\right) .
$$

We need to show that under these assumptions

(1) $\forall y_{1}^{*}, y_{1}, \ldots, y_{n}^{*}, y_{n}\left(\bigwedge_{i=1}^{n} y_{i}^{*} \gtrsim_{\rho_{i}}^{a} y_{i} \rightarrow x^{*} y_{1}^{*} \ldots y_{n}^{*} \gtrsim_{\tau_{0}}^{a} x y_{1} \ldots y_{n}\right)$ and

(2) $\forall y_{1}^{*}, y_{1}, \ldots, y_{n}^{*}, y_{n}\left(\bigwedge_{i=1}^{n} y_{i}^{*} \gtrsim_{\widehat{\rho}_{i}}^{a} y_{i} \rightarrow x^{*} y_{1}^{*} \ldots y_{n}^{*} \gtrsim_{\tau_{0}}^{a} x^{*} y_{1} \ldots y_{n}\right)$ hold. Then using the induction hypothesis we are done.

There are four cases to check:

$$
\begin{aligned}
& \text { (1a) } \forall y_{1}^{*}, y_{1}, \ldots, y_{n+1}^{*}, y_{n+1}\left(\bigwedge_{i=1}^{n+1} y_{i}^{*} \gtrsim_{\rho_{i}}^{a} y_{i} \rightarrow\left(x^{*} y_{1}^{*} \ldots y_{n}^{*}\right) y_{n+1}^{*}\right. \\
& \left.\gtrsim_{\tau}^{a}\left(x y_{1} \ldots y_{n}\right) y_{n+1}\right), \\
& \text { (1b) } \forall y_{1}^{*}, y_{1}, \ldots, y_{n+1}^{*}, y_{n+1}\left(\bigwedge_{i=1}^{n} y_{i}^{*} \gtrsim_{\rho_{i}}^{a} y_{i} \wedge y_{n+1}^{*} \gtrsim_{\hat{\rho}_{n+1}}^{a} y_{n+1} \rightarrow\left(x^{*} y_{1}^{*} \ldots y_{n}^{*}\right) y_{n+1}^{*}\right. \\
& \left.\gtrsim_{\widehat{\tau}}^{a}\left(x^{*} y_{1}^{*} \ldots y_{n}^{*}\right) y_{n+1}\right), \\
& \text { (2a) } \forall y_{1}^{*}, y_{1}, \ldots, y_{n+1}^{*}, y_{n+1}\left(\bigwedge_{i=1}^{n+1} y_{i}^{*} \gtrsim_{\hat{\rho}_{i}}^{a} y_{i} \rightarrow\left(x^{*} y_{1}^{*} \ldots y_{n}^{*}\right) y_{n+1}^{*}\right. \\
& \left.\gtrsim_{\widehat{\tau}}^{a}\left(x^{*} y_{1} \ldots y_{n}\right) y_{n+1}\right), \\
& \text { (2b) } \forall y_{1}^{*}, y_{1}, \ldots, y_{n+1}^{*}, y_{n+1}\left(\bigwedge_{i=1}^{n+1} y_{i}^{*} \gtrsim_{\widehat{\rho}_{i}}^{a} y_{i} \rightarrow\left(x^{*} y_{1}^{*} \ldots y_{n}^{*}\right) y_{n+1}^{*}\right. \\
& \left.\gtrsim_{\widehat{\tau}}^{a}\left(x^{*} y_{1}^{*} \ldots y_{n}^{*}\right) y_{n+1}\right) .
\end{aligned}
$$

(1a) and (2a) hold by assumption, (1b) and (2b) follow from (2a) using Lemma 9.5 .

In the next lemma (whose easy proof we omit) we need the (primitive recursive) maximum functional for sequences of objects of a common type in $\mathbf{T}$ from Definition 4.9 which allows one to construct majorants out of pointwise majorants for objects having a sequence type $0 \rightarrow \rho$, where $\rho \in \mathbf{T}^{X}$ (note that the pointwise majorizing functional then has the type $0 \rightarrow \widehat{\rho}$ with $\widehat{\rho} \in \mathbf{T})$.

Lemma 9.7. Let $\rho \in \mathbf{T}^{X}$ and $x^{*} \in M_{\widehat{\rho}}^{M_{0}}, x \in M_{\rho}^{M_{0}}$. If $\forall n\left(x^{*}(n) \gtrsim_{\rho}^{a} x(n)\right)$, then $\left(x^{*}\right)^{M} \gtrsim_{0 \rightarrow \rho}^{a} x \in M_{0 \rightarrow \rho}$.

We now prove Theorem 4.10. We focus on hyperbolic spaces $(X, d, W)$ and the corresponding theory $\mathcal{A}^{\omega}[X, d, W]_{-b}$. The case of ordinary metric spaces $(X, d)$ follows by simply omitting the axioms concerning $W$, while for CAT(0)-spaces we merely need to consider the additional purely universal axiom $\mathbf{C N}^{-}$. The next lemma is an adaptation of the corresponding result from [25] and states that $\mathcal{A}^{\omega}[X, d, W]_{-b}$ has (via its so-called negative translation) a Gödel functional interpretation in $\mathcal{A}^{\omega}[X, d, W]_{-b} \backslash\{\mathrm{QF}-\mathrm{AC}\}$ (actually even without $\mathrm{DC}$ and in a quantifier-free fragment of this theory) augmented by the schema (BR) of simultaneous bar recursion in all types of $\mathbf{T}^{X}$ (for $\mathcal{A}^{\omega}$ this fundamental result is due to [40] (see [33] for a comprehensive treatment) which extends [10], where functional interpretation was introduced). 
Let $\mathcal{A}^{\omega}[X, d, W]_{-b}^{-}:=\mathcal{A}^{\omega}[X, d, W]_{-b} \backslash\{\mathrm{QF}-\mathrm{AC}\}$.

Analogously, we define $\mathcal{A}^{\omega}[X,\|\cdot\|, C]_{-b}^{-}:=\mathcal{A}^{\omega}[X,\|\cdot\|, C]_{-b} \backslash\{\mathrm{QF}-\mathrm{AC}\}$, etc.

Lemma 9.8. Let $A$ be a sentence in the language of $\mathcal{A}^{\omega}[X, d, W]_{-b}$. Then the following rule holds:

$$
\left\{\begin{array}{l}
\mathcal{A}^{\omega}[X, d, W]_{-b} \vdash A \\
\Rightarrow \text { one can construct a tuple of closed terms } \underline{t} \text { of } \mathcal{A}^{\omega}[X, d, W]_{-b}+(\mathrm{BR}) \text { s.t. } \\
\mathcal{A}^{\omega}[X, d, W]_{-b}^{-}+(\mathrm{BR}) \vdash \forall \underline{y}\left(A^{\prime}\right)_{D}(\underline{t}, \underline{y}),
\end{array}\right.
$$

where $A^{\prime}$ is the negative translation of $A$ and $\left(A^{\prime}\right)^{D} \equiv \exists \underline{x} \forall \underline{y}\left(A^{\prime}\right)_{D}(\underline{x}, \underline{y})$ is the Gödel functional interpretation of $A^{\prime}$.

Proof. This is Lemma 4.4 in [25] (see also the correction at the end of [9]), except that we have one purely universal axiom less to interpret: the axiom that the metric of $(X, d, W)$ is bounded by $b$. Also, as discussed in section 2 the axioms concerning $W_{X}$ have been reformulated to implicitly satisfy $W_{X}(x, y, \lambda)={ }_{X} W_{X}(x, y, \tilde{\lambda})$. Recall that, in general, purely universal axioms not containing $\vee$, such as the additional axioms for metric, hyperbolic and CAT(0)-spaces, are interpreted by themselves.

Lemma 9.9. Let $(X, d, W)$ be a nonempty hyperbolic space. Then $\mathcal{M}^{\omega, X}$ is a model of $\mathcal{A}^{\omega}[X, d, W]_{-b}^{-}+(\mathrm{BR})$ (for a suitable interpretation of the constants of $\mathcal{A}^{\omega}[X, d, W]_{-b}^{-}+(\mathrm{BR})$ in $\left.\mathcal{M}^{\omega, X}\right)$, where we may interpret $0_{X}$ by an arbitrary element $a \in X$.

Moreover, for any closed term $t$ of $\mathcal{A}^{\omega}[X, d, W]_{-b}^{-}+(\mathrm{BR})$ one can construct a closed term $t^{*}$ of $\mathcal{A}^{\omega}+(\mathrm{BR})$ - so, in particular, $t^{*}$ does not contain the constants $0_{X}, d_{X}$ and $W_{X}-$ such that

$$
\mathcal{M}^{\omega, X} \models \forall a^{X} \forall n^{0}\left((n)_{\mathbb{R}} \geq d\left(0_{X}, a\right) \rightarrow t^{*}(n) \gtrsim^{a} t\right) .
$$

In particular, if we interpret $0_{X}$ by $a \in X$, then it holds in $\mathcal{M}^{\omega, X}$ that $t^{*}\left(0^{0}\right)$ is an a-majorant of $t$ (note that $t^{*}\left(0^{0}\right)$ does not depend on a).

Proof. The constants of $\mathcal{A}^{\omega}[X, d, W]_{-b}^{-}+(\mathrm{BR})$ are interpreted as in [25], so next we need to show that all these functionals are in $\mathcal{M}^{\omega, X}$ by constructing $a$-majorants. To show that we can construct a suitable $a$-majorant $t^{*}$ for any closed term $t$ of $\mathcal{A}^{\omega}[X, d, W]_{-b}^{-}+(\mathrm{BR})$ it suffices to describe $a$-majorants for the constants of $\mathcal{A}^{\omega}[X, d, W]_{-b}^{-}+(\mathrm{BR})$.

We first describe the (trivially uniform) $a$-majorants for the constants of classical analysis $\mathcal{A}^{\omega}$, which now are taken over the extended set of types $\mathbf{T}^{X}$. Using Lemma 9.6 one easily verifies that:

- $0 \gtrsim_{0}^{a} 0$,

- $S \gtrsim_{1}^{a} S$,

- $\Pi_{\widehat{\rho}, \widehat{\tau}} \gtrsim^{a} \Pi_{\rho, \tau}$,

- $\Sigma_{\widehat{\sigma}, \widehat{\rho}, \widehat{\tau}} \gtrsim^{a} \Sigma_{\sigma, \rho, \tau}$.

To produce $a$-majorants for the (simultaneous) recursor(s) $\underline{R}$ and the (simultaneous) bar-recursor(s) $\underline{B},{ }^{26}$ we only need the functional $\max _{\rho}$ defined for all types

\footnotetext{
${ }^{26}$ As in [25], our formal systems are formulated with simultaneous recursion $\underline{R}$ and simultaneous bar-recursion $\underline{B}$, both of which could be defined primitive recursively in ordinary recursion $R$ and ordinary bar-recursion $B$ on the presence of appropriate product types. For convenience we suppress the tuple notation in the following. See [25] for a detailed discussion.
} 
$\rho \in \mathbf{T}$. As $a$-majorants for $R$ and $B$ only operate on the types $\mathbf{T}$, we do not need to extend $\max _{\rho}$ to the types $\mathbf{T}^{X}$ as it was done in [25].

By induction on $n$ and using Lemma 9.6 one easily proves $\forall n\left(R_{\widehat{\rho}} n \gtrsim_{\rho}^{a} R_{\rho} n\right)$ and hence by Lemma $9.7 R_{\widehat{\rho}}^{M} \gtrsim_{\rho}^{a} R_{\rho}$.

The majorant for the bar-recursor $B_{\rho, \tau}$ is defined as

$$
B_{\widehat{\rho}, \widehat{\tau}}^{*}:=\lambda x, z, u, n, y \cdot\left(B_{\widehat{\rho}, \widehat{\tau}} x^{M} z^{M} u_{z}\right)^{M} n y,
$$

where $x^{M}\left(y^{0 \rightarrow \widehat{\rho}}\right):=x\left(y^{M}\right), z^{M} n y:=z n y^{M}$ and $u_{z}:=\lambda v, n, y \cdot \max \left(z n y^{M}, u v n y^{M}\right)$. As the defining axioms of $B$ involve $0_{X}$, we here assume $a=0_{X}$, though by Lemma 9.3 given a bound on $d\left(a, 0_{X}\right)$ we may transform this majorant into a majorant for any choice of $a$. If $0_{X}$ does not occur, such that we may interpret $0_{X}$ by $a \in X$, the dependency on $0_{X}$, resp. a bound on $d\left(a, 0_{X}\right)$, disappears.

The crucial step in proving $B_{\widehat{\rho}, \widehat{\tau}}^{*} \gtrsim^{0_{X}} \quad B_{\rho, \tau}$ is to establish the following: Let $x^{*}, z^{*}, u^{*}, x, z, u, \widehat{x}, \widehat{z}, \widehat{u}$ be given s.t. $x^{*} \gtrsim_{\alpha} x, x^{*} \gtrsim_{\widehat{\alpha}} \widehat{x}, z^{*} \gtrsim_{\beta} z, z^{*} \gtrsim_{\widehat{\beta}} \widehat{z}, u^{*} \gtrsim_{\gamma} u$ and $u^{*} \gtrsim_{\widehat{\gamma}} \widehat{u}$, where $\alpha, \beta$ and $\gamma$ are determined by $\rho$ and $\tau$. Then

$$
\forall y \in M_{0 \rightarrow \widehat{\rho}} \forall n^{0} Q(\overline{y, n} ; n),
$$

where $Q\left(y_{i} n\right): \equiv$

$$
\left\{\begin{array}{l}
\forall y^{*}, \tilde{y}, \widehat{\tilde{y}} \in \mathcal{M}^{\omega, X}\left(\forall k\left(y^{*} k \gtrsim_{\rho} \tilde{y} k \wedge y^{*} k \gtrsim_{\widehat{\rho}} \widehat{\tilde{y}} k\right) \wedge \overline{y^{*}, n}=_{0 \rightarrow \widehat{\rho}} \overline{y, n}\right. \\
\quad \Rightarrow B^{+} x^{*} z^{*} u^{*} n y^{*} \gtrsim_{\tau} B x z u n \tilde{y} \\
\left.\wedge B^{+} x^{*} z^{*} u^{*} n y^{*} \gtrsim_{\widehat{\tau}} B^{+} x^{*} z^{*} u^{*} n \widehat{\tilde{y}}, B^{+} \widehat{x} \widehat{z} \widehat{u} n \widehat{\tilde{y}}\right)
\end{array}\right.
$$

with $B_{\rho, \tau}^{+}:=\lambda x, z, u, n, y \cdot B_{\rho, \tau} x^{M} z^{\mu} u_{z} n y$. By Lemmas 9.6 and 9.7 it then follows that $B^{*} \gtrsim B$ and $B^{*}, B \in \mathcal{M}^{\omega, X}$.

The proof of $(+)$ uses the following form of dependent choice, also called bar induction (which holds in $\mathcal{M}^{\omega, X}$ since by Lemma 9.7 we have $M_{0 \rightarrow \rho}=\left(M_{\rho}\right)^{\mathbb{N}}$ ):

$$
\left\{\begin{array}{l}
\forall y \in M_{0 \rightarrow \widehat{\rho}} \exists n_{0} \in \mathbb{N} \forall n \geq n_{0} Q(\overline{y, n} ; n) \\
\wedge \forall y \in M_{0 \rightarrow \widehat{\rho}}, n \in \mathbb{N}\left(\forall D \in M_{\widehat{\rho}} Q(\overline{y, n} * D ; n+1) \rightarrow Q(\overline{y, n} ; n)\right) \\
\rightarrow \forall y \in M_{0 \rightarrow \widehat{\rho}}, n \in \mathbb{N} Q(\overline{y, n} ; n) .
\end{array}\right.
$$

For the additional constants of $\mathcal{A}^{\omega}[X, d, W]_{-b}^{-}$we define the following $a$-majorants:

- $n^{0} \gtrsim^{a} 0_{X}$ for every $n$ with $(n)_{\mathbb{R}} \geq_{\mathbb{R}} d_{X}\left(a, 0_{X}\right)$, where as just mentioned we can take $n:=0$ if we interpret $0_{X}$ by $a$,

- $0^{0} \gtrsim^{a} a$, since $d_{X}(a, a)={ }_{\mathbb{R}}(0)_{\mathbb{R}}$,

- $\lambda x^{0}, y^{0} \cdot\left((x+y)_{\mathbb{R}}\right)_{\circ} \gtrsim^{a} d_{X}^{X \rightarrow X \rightarrow 1},{ }^{27}$

- $\lambda x^{0}, y^{0}, z^{1} \cdot \max _{0}(x, y) \gtrsim^{a} W_{X}^{X \rightarrow X \rightarrow 1 \rightarrow X}$.

The $a$-majorants for $0_{X}$ and $a$ are obvious. The $a$-majorant for $d_{X}$ follows from the triangle inequality: assume $n_{1} \gtrsim^{a} x$ and $n_{2} \gtrsim^{a} y$; then

$$
d(x, y) \leq d(x, a)+d(y, a) \leq n_{1}+n_{2} .
$$

Hence, as in the model $\mathcal{M}^{\omega, X}$ the expression $d_{X}(x, y)$ is interpreted by $(d(x, y))$ 。 (see [25]) and by Lemma $4.6 n_{1}+n_{2} \geq d(x, y)$ implies $\left(\left(n_{1}+n_{2}\right)_{\mathbb{R}}\right)_{\circ}$ s-maj $_{1}(d(x, y))_{\circ}$, the validity of the $a$-majorant for $d_{X}$ follows.

Finally, the $a$-majorant for $W_{X}$ can be justified by the first axiom for hyperbolic spaces:

$$
\forall x^{X}, y^{X}, z^{X} \forall \lambda^{1}\left(d_{X}\left(z, W_{X}(x, y, \lambda)\right) \leq_{\mathbb{R}}\left(1-_{\mathbb{R}} \tilde{\lambda}\right) d_{X}(z, x)+_{\mathbb{R}} \tilde{\lambda} d_{X}(z, y)\right) .
$$

\footnotetext{
${ }^{27}$ Here we refer to Remark 4.5.
} 
The construction $\tilde{\lambda}$ turns representatives $\lambda$ of arbitrary real numbers into representatives $\tilde{\lambda}$ of real numbers in the interval $[0,1]$. Hence we may reason that $d_{X}\left(z, W_{X}(x, y, \lambda)\right)$ is less than the maximum of $d_{X}(z, x)$ and $d_{X}(z, y)$ and hence less than the maximum of respective upper bounds on $d_{X}(z, x)$ and $d_{X}(z, y)$. Note that without the reformulation of axioms (4)-(7) for hyperbolic spaces discussed in section 2 (see Remark 2.6), this reasoning only holds in the model $\mathcal{M}^{\omega, X}$ (in which $W_{X}(x, y, \lambda)$ is interpreted by $W\left(x, y, r_{\tilde{\lambda}}\right)$, where $W$ is the function of the hyperbolic space $(X, d, W)$ and $r_{\tilde{\lambda}}$ is the real number in $[0,1]$ represented by $\left.\tilde{\lambda}\right)$, whereas now it is even provable in $\mathcal{A}^{\omega}[X, d, W]_{-b}$.

Note that the $a$-majorants for $d_{X}, W_{X}$ are uniform, i.e. they do not depend on $a$. The $a$-majorant for $a$ also does not depend on $a$, other than the requirement that the variable $a$ and the element $a$ in $\gtrsim^{a}$ denote the same element. Only the $a$ majorant for $0_{X}$ depends on $a$. Also note that the $(\cdot)_{\circ}$-operator, which is ineffective in general, only is applied to natural numbers, where it is effectively (even primitive recursively) computable.

Thus given a closed term $t$ of $\mathcal{A}^{\omega}[X, d, W]_{-b}^{-}+(\mathrm{BR})$ we construct an $a$-majorant $t^{*}$ by induction on the term structure from the $a$-majorants given above, where we furthermore $\lambda$-abstract the majorant $n$ for $0_{X}$. Then one easily shows that

$$
\mathcal{M}^{\omega, X} \models \forall a^{X} \forall n^{0}\left((n)_{\mathbb{R}} \geq_{\mathbb{R}} d_{X}\left(0_{X}, a\right) \rightarrow t^{*}(n) \gtrsim^{a} t\right),
$$

where $t^{*}$ does not contain $0_{X}, d_{X}$ and $W_{X}$ and we may take $n:=0$ if we interpret $0_{X}$ by $a$. Here we use the fact that for elements in $\mathcal{M}^{\omega, X}$ the interpretation of the syntactic relation $\gtrsim^{a}$ in this model coincides with the semantic relation used to define the model (note that $(n)_{\mathbb{R}} \geq_{\mathbb{R}}(d(x, a))_{\circ}$ is equivalent to $\left.n \geq d(x, a)\right)$.

Lemma 9.9 also covers $\mathcal{A}^{\omega}[X, d]_{-b}$, simply by omitting the parts concerning the $W$-function, and $\mathcal{A}^{\omega}[X, d, W, \mathrm{CAT}(0)]_{-b}$, as this theory contains no additional constants that need to be majorized but only another purely universal axiom which is interpreted by itself.

\section{Proof of Theorem 4.10.}

Assume

$$
\mathcal{A}^{\omega}[X, d, W]_{-b} \vdash \forall x^{\rho}\left(\forall u^{0} B_{\forall}(x, u) \rightarrow \exists v^{0} C_{\exists}(x, v)\right) .
$$

As in [25], this yields (using the fact that negative translation and (partial) functional interpretation of the formula in question results in $\exists U, V \forall x^{\rho}\left(B_{\forall}(x, U(x)) \rightarrow\right.$ $\left.\left.C_{\exists}(x, V(x))\right)\right)$ by Lemmas 9.8 and 9.9 the extractability of closed terms $t_{U}, t_{V}$ of $\mathcal{A}^{\omega}[X, d, W]+(\mathrm{BR})$ and closed terms $t_{U^{*}}, t_{V^{*}}$ of $\mathcal{A}^{\omega}+(\mathrm{BR})$ (in particular, $t_{U^{*}}, t_{V^{*}}$ do not contain $0_{X}, d_{X}, W_{X}$ and the other constants only for types in $\mathbf{T}$ ) such that for all $n^{0} \geq d\left(0_{X}, a\right)$

$$
\mathcal{M}^{\omega, X} \models\left\{\begin{aligned}
t_{U^{*}}(n) \gtrsim^{a} t_{U} \wedge t_{V^{*}}(n) & \gtrsim^{a} t_{V} \\
\wedge \forall x^{\rho}\left(B_{\forall}\left(x, t_{U}(x)\right)\right. & \left.\rightarrow C_{\exists}\left(x, t_{V}(x)\right)\right) .
\end{aligned}\right.
$$

Next, define the functional $\Phi\left(x^{\widehat{\rho}}, n\right):=\max \left(t_{U^{*}}(n, x), t_{V^{*}}(n, x)\right)$. Then

$$
\mathcal{M}^{\omega, X} \models \forall u \leq \Phi\left(x^{*}, n\right) B_{\forall}(x, u) \rightarrow \exists v \leq \Phi\left(x^{*}, n\right) C_{\exists}(x, v)
$$

holds for all $n \in \mathbb{N}, x \in M_{\rho}$ and $x^{*} \in M_{\widehat{\rho}}$ for which there exists an $a \in X$ such that $n \geq d\left(0_{X}, a\right)$ and $x^{*} \gtrsim^{a} x$. We now show that $(+)$ also holds in $\mathcal{S}^{\omega, X}$ : For the types $\gamma$ of degree $\widehat{1}$ or $(1, X)$ of the quantifiers hidden in the definition of $\forall / \exists$-formulas we have at least $M_{\gamma} \subseteq S_{\gamma}$, which is sufficient for our purposes. This is because types of that kind have arguments for whose types $\delta$ one has - using Lemma 9.7 - that 
$M_{\delta}=S_{\delta}$. For parameters $x^{\rho}$ with $\rho$ of degree 2 or $(1, X)$, we restricted ourselves to those $x \in S_{\rho}$ which have $a$-majorants $x^{*} \in S_{\widehat{\rho}}$. Since functionals of such types $\rho$ again only have arguments of types $\tau$ for which $M_{\tau}=S_{\tau}$, we get from $x^{*} \gtrsim_{\rho}^{a} x$ (which implies that $x^{*} \gtrsim_{\widehat{\rho}}^{a} x^{*}$ ) that $x^{*} \in M_{\widehat{\rho}}, x \in M_{\rho}$. Hence $\Phi\left(x^{*}, n\right)$ is defined and $(+)$ yields that

$(++) \quad \mathcal{S}^{\omega, X} \models \forall u \leq \Phi\left(x^{*}, n\right) B_{\forall}(x, u) \rightarrow \exists v \leq \Phi\left(x^{*}, n\right) C_{\exists}(x, v)$

holds for all $n \in \mathbb{N}, x \in S_{\rho}$ and $x^{*} \in S_{\widehat{\rho}}$ for which there exists an $a \in X$ such that $n^{0} \geq d\left(0_{X}, a\right)$ and $x^{*} \gtrsim^{a} x$ (here $\Phi\left(x^{*}, n\right)$ is interpreted as $\left.\left[\Phi\left(x^{*}, n\right)\right]_{\mathcal{M}^{\omega}}\right)$. Here we use the fact that for closed terms $t^{\eta}$ of $\mathcal{A}^{\omega}[X, d, W]_{-b}$ we have $[t]_{\mathcal{M}^{\omega, X}} \approx_{\eta}[t]_{\mathcal{S}^{\omega, X}}$, where

$$
\begin{aligned}
& x \approx_{0} y: \equiv x, y \in \mathbb{N} \wedge x=y, x \approx_{X} y: \equiv x, y \in X \wedge x=y, \\
& x \approx_{\rho \rightarrow \tau} y: \equiv x \in M_{\rho \rightarrow \tau}, y \in S_{\rho \rightarrow \tau} \wedge \forall u \in M_{\rho}, v \in S_{\rho}\left(u \approx_{\rho} v \rightarrow x u \approx_{\tau} y v\right) .
\end{aligned}
$$

This is easily proved for the constants and hence for the closed terms.

This finishes the proof, as $\Phi$ is a partial (bar recursive) functional in $S_{\widehat{\rho} \rightarrow 0 \rightarrow 0}$ (which is always defined on the majorizable elements $M_{\widehat{\rho}}$ of $S_{\widehat{\rho}}$ and whose restriction to $M_{\widehat{\rho}}$ is computable (in the sense of Kleene's S1-S9 relativised to $\mathcal{M}^{\omega}$ ). If in addition to being of degree $(1, X)$ the type $\rho$ is of degree $\widehat{1}$, then $\Phi$ is total since then $M_{\widehat{\rho}}=S_{\widehat{\rho}}$.

Finally, if $0_{X}$ does not occur in either $B_{\forall}$ or $C_{\exists}$ we may freely interpret $0_{X}$ by $a \in X$. We then get majorants $t_{U^{*}}, t_{V^{*}}$ and a resulting term $\Phi$ which no longer depends on a bound $n$ on $d\left(0_{X}, a\right)$ (as we can take $n:=0$ ).

Remark 9.10. The proof of the soundness theorem of Gödel's functional interpretation (by closed terms) requires that we have closed terms for each type which we can use e.g. in order to construct the functional interpretation of axioms such as $\perp \rightarrow A$. That closed term can be arbitrarily chosen and is usually taken as the constant-0-functional of suitable type, where for the type $X$ one takes by default $0_{X}$. However, one could also have chosen an open term such as the constant-afunctional for types which map arguments to the type $X$. So rather than having just one term extracted by functional interpretation we have a whole family of such terms parametrized by $a^{X}$. In the last step of the previous proof we make use of this by picking the $a$-th term according to our choice of the reference point $a$ for $\gtrsim^{a}$. By letting both $\gtrsim^{a}$ and the functional interpretation depend on $a$ in a simultaneous way we achieve that the extracted majorant does not depend on the distance between $a$ and any arbitrarily fixed constant such as $0_{X}$.

For normed linear spaces we focus on the theory $\mathcal{A}^{\omega}[X,\|\cdot\|, C]_{-b}$. The further cases $\mathcal{A}^{\omega}[X,\|\cdot\|, C, \eta]_{-b}$ and $\mathcal{A}^{\omega}[X,\langle\cdot, \cdot\rangle, C]_{-b}$ follow by extending $\mathcal{A}^{\omega}[X,\|\cdot\|, C]_{-b}$ respectively with an additional (majorizable) constant $\eta$ for the modulus of uniform convexity or an additional purely universal axiom expressing the properties of the inner product in terms of the norm.

Lemma 9.11. Let $(X,\|\cdot\|)$ be a nontrivial real normed linear space with a nonempty convex subset $C$. Then $\mathcal{M}^{\omega, X}$ is a model of $\mathcal{A}^{\omega}[X,\|\cdot\|, C]_{-b}^{-}+(\mathrm{BR})$ (for a suitable interpretation of the constants of $\mathcal{A}^{\omega}[X,\|\cdot\|, C]_{-b}+(\mathrm{BR})$ in $\mathcal{M}^{\omega, X}$ where we have to interpret $0_{X}$ by the zero vector 0 in $X$ and use $\left.\gtrsim^{0_{X}}\right)$. 
Moreover, for any closed term $t$ of $\mathcal{A}^{\omega}[X,\|\cdot\|, C]_{-b}+(\mathrm{BR})$ one can construct a closed term $t^{*}$ of $\mathcal{A}^{\omega}+(\mathrm{BR})$ such that

$$
\mathcal{M}^{\omega, X} \models \forall n^{0}\left((n)_{\mathbb{R}} \geq_{\mathbb{R}}\left\|c_{X}\right\|_{X} \rightarrow t^{*}(n) \gtrsim^{0_{X}} t\right) .
$$

Similarly for $\mathcal{A}^{\omega}[X,\|\cdot\|, C, \eta]_{-b}^{-}+(\mathrm{BR})$ and $\mathcal{A}^{\omega}[X,\langle\cdot, \cdot\rangle, C]_{-b}^{-}+(\mathrm{BR})$.

Proof. The proof is almost the same as the above proof of Lemma 9.9, and as before the interpretation of the constants of $\mathcal{A}^{\omega}[X,\|\cdot\|, C]_{-b}+(\mathrm{BR})$ is as in [25].

The main difference to the proof of Lemma 9.9 is that we fix $a=0_{X}$ (where $0_{X}$ now has to be interpreted by the zero vector of $X$ ), as otherwise we cannot define suitable (i.e. suitably uniform) majorants for the new constants of $\mathcal{A}^{\omega}[X,\|\cdot\|, C]_{-b}$. So now it suffices to state $0_{X}$-majorants for the new constants:

- $0^{0} \gtrsim^{0} 0_{X}$,

- $1^{0} \gtrsim^{0}{ }^{0} 1_{X}$,

- $\lambda x^{0} \cdot\left((x)_{\mathbb{R}}\right)_{\circ} \gtrsim^{0_{X}}\|\cdot\|_{X}^{X \rightarrow 1}$

- $\lambda x^{0}, y^{0} \cdot x+y \underset{z_{X}}{0_{X}}+\underset{X}{X} \rightarrow X \rightarrow X$,

- $\lambda x^{0} \cdot x \gtrsim^{0}-\underset{X}{\widetilde{X} \rightarrow X}$,

- $\lambda \alpha^{1}, x^{0} \cdot(\alpha(0)+1) \cdot x \gtrsim^{0} X \cdot{ }_{X}^{1 \rightarrow X \rightarrow X}$.

For the convex subset $C$, we have the characteristic term $\chi_{C}$ for the subset $C$, which is majorized as follows:

$$
\lambda x^{0} .1 \gtrsim^{0_{X}} \chi_{C}^{X \rightarrow 0} .
$$

For the constant $c_{X} \in C$ we have, given an $n \geq\left\|c_{X}\right\|$, the $0_{X}$-majorant

$$
n^{0} \gtrsim_{X}^{0} c_{X}
$$

For uniformly convex spaces we $0_{X}$-majorize the modulus $\eta: \mathbb{N} \rightarrow \mathbb{N}$ of uniform convexity by

$$
(\eta)^{M} \gtrsim_{1}^{0_{X}} \eta
$$

In [25], inner product spaces are defined by adding the so-called parallelogram law as another axiom. A norm satisfying the parallelogram law allows one to define an inner product in terms of the norm and hence the inner product is immediately $0_{X}$-majorizable. Conversely, if only an inner product is given the norm $\|\cdot\|$ can be recovered by defining $\|x\|:=\sqrt{\langle x, x\rangle}$.

The majorants for the constants of normed linear spaces, with the exception of the modulus $\eta$ of uniform convexity, are only seemingly uniform, since they depend on properties of the norm and hence on the choice of $a=0_{X}$. The $0_{X}$-majorants for $0_{X}, 1_{X}, \chi_{C}$ and $c_{X}$ are obvious. For $\|\cdot\|_{X}$ we need to consider the interpretation of $\|\cdot\|_{X}$ in the model $\mathcal{M}^{\omega, X}$ : the norm $\|x\|_{X}$ of an element $x \in X$ is interpreted by the actual norm using the ()$_{\circ}$-operator, i.e. by $(\|x\|)_{\circ}$. In order to show that (in the model) $\lambda x .\left((x)_{\mathbb{R}}\right)_{\circ} \gtrsim^{a}\|\cdot\|_{X}$ we need to show two things: (1) if $n \gtrsim^{0_{X}} x$, then $\left((n)_{\mathbb{R}}\right) \circ \mathrm{s}^{-\mathrm{maj}_{1}}(\|x\|) \circ$ and $(2)$ if $n \geq m$, then $\left((n)_{\mathbb{R}}\right)_{\circ} \mathrm{s}^{-\mathrm{maj}_{1}}\left((m)_{\mathbb{R}}\right)$ 。 (recall that for $\rho \in \mathbf{T ~ s - m a j} \rho$ and $\gtrsim_{\rho}^{a}$ are equivalent). For (1), if $n \gtrsim^{0_{X}} x$, then by definition $(n)_{\mathbb{R}} \geq_{\mathbb{R}}\|x\|_{X}$ and the result then follows by Lemma 4.6. For (2) the result follows directly from Lemma 4.6 (or even more easily from the definition of $\left((n)_{\mathbb{R}}\right)$ 。 as given in Remark 4.5). For $-_{X}$ the $0_{X}$-majorant is derived straightforwardly from basic properties of the norm $\|\cdot\|_{X}$. For $+_{X}$ we additionally use the triangle inequality to verify the majorant, i.e. $\|x+y\| \leq\|x\|+\|y\|$, and then if $n_{1} \gtrsim^{0_{X}} x$ and $n_{2} \gtrsim^{0_{X}} y$ we have that $n_{1}+n_{2} \geq\|x+y\|$, and the validity of the majorant follows. 
Finally, for scalar multiplication $\cdot_{X}$ we use the fact that $\alpha$ codes a real number via a Cauchy sequence of rational numbers with fixed rate of convergence. The rational numbers in turn are represented by natural numbers using a monotone coding function such that $(\alpha(n))_{\mathbb{Q}} \geq_{\mathbb{Q}}|\alpha(n)|_{\mathbb{Q}}$ for all $n$. Since $\left|\lambda n^{0} . \alpha(0)-\mathbb{R} \alpha\right| \leq 1$ the natural number $\alpha(0)+1$ is an upper bound for the real number represented by $|\alpha|_{\mathbb{R}}$. Now let $\alpha^{*} \mathrm{~s}^{-\mathrm{maj}_{1}} \alpha$. Then $\alpha^{*}(0)+1 \geq \alpha(0)+1$. Since $\|\alpha \cdot x\|_{X}=_{\mathbb{R}}|\alpha|_{\mathbb{R}} \cdot \mathbb{R}\|x\|_{X}$ we, therefore, have that $\alpha^{*}(0)+1$ taken as a natural number multiplied with an $n \gtrsim^{0_{X}} x$ is a $0_{X}$-majorant for $\alpha \cdot{ }_{X} x$.

Proof of Theorem 6.3. As in the proof of Theorem 4.10

$$
\mathcal{A}^{\omega}[X,\|\cdot\|, C]_{-b} \vdash \forall x^{\rho}\left(\forall u^{0} B_{\forall}(x, u) \rightarrow \exists v^{0} C_{\exists}(x, v)\right)
$$

yields (using an easy adaptation of Lemma 9.8 and Lemma 9.11) the extractability of closed terms $t_{U}, t_{V}$ of $\mathcal{A}^{\omega}[X,\|\cdot\|, C]_{-b}+(\mathrm{BR})$ and closed terms $t_{U^{*}}, t_{V^{*}}$ of $\mathcal{A}^{\omega}+(\mathrm{BR})$ (so, in particular, $t_{U^{*}}, t_{V^{*}}$ do not contain $0_{X}, 1_{X}, c_{X},+_{X},-_{X},{ }_{X}, \chi_{C}$ or $\left.\|\cdot\|_{X}\right)$ such that for all $n^{0} \geq\left\|c_{X}\right\|$

$$
\mathcal{M}^{\omega, X} \models\left\{\begin{array}{c}
t_{U^{*}}(n) \underset{0^{0}}{\gtrsim^{\rho}} t_{U} \wedge t_{V^{*}}(n) \gtrsim^{0_{X}} t_{V} \\
\wedge \forall x^{\rho}\left(B_{\forall}\left(x, t_{U}(x)\right) \rightarrow C_{\exists}\left(x, t_{V}(x)\right)\right) .
\end{array}\right.
$$

As before, defining $\Phi\left(x^{\widehat{\rho}}, n\right):=\max \left(t_{U^{*}}(n, x), t_{V^{*}}(n, x)\right)$, we then have that

$$
\mathcal{M}^{\omega, X} \models \forall u \leq \Phi\left(x^{*}, n\right) B_{\forall}(x, u) \rightarrow \exists v \leq \Phi\left(x^{*}, n\right) C_{\exists}(x, v)
$$

holds for all $n \in \mathbb{N}, x \in M_{\rho}$ and $x^{*} \in M_{\widehat{\rho}}$ for which $n \geq\left\|c_{X}\right\|$ and $x^{*} \gtrsim^{0 x} x$.

Also for normed linear spaces one verifies that for the types $\gamma$ of degree $\hat{1}$ and $(1, X)$ hidden in the definition of $\forall / \exists$-formulas, we have the necessary inclusion $M_{\gamma} \subseteq S_{\gamma}$. For parameters $x^{\rho}$ with types $\rho$ of degree $(1, X),(1, X, C)$ or 2 , we again restrict ourselves to functionals which have $0_{X}$-majorants $x^{*}$ and hence the necessary inclusions hold.

Thus, also

$$
\mathcal{S}^{\omega, X} \models \forall u \leq \Phi\left(x^{*}, n\right) B_{\forall}(x, u) \rightarrow \exists v \leq \Phi\left(x^{*}, n\right) C_{\exists}(x, v)
$$

holds for all $n \in \mathbb{N}, x \in S_{\rho}$ and $x^{*} \in S_{\widehat{\rho}}$ for which $n^{0} \geq\left\|c_{X}\right\|$ and $x^{*} \gtrsim^{0 x} x$. In uniformly convex spaces the bound additionally depends on a modulus $\eta$ of uniform convexity via its majorant $\eta^{M}$.

This finishes the proof as in the proof of Theorem 4.10.

\section{REFERENCES}

1. M. Bezem, Strongly majorizable functionals of finite type: a model of bar recursion containing discontinous functionals, J. of Symbolic Logic 50 (1985), 652-660. MR805674 (87c:03139)

2. J. M. Borwein, S. Reich, and I. Shafrir, Krasnoselski-Mann iterations in normed spaces, Canad. Math. Bull. 35 (1992), 21-28. MR1157459 (93e:47072)

3. M. R. Bridson and A. Haefliger, Metric spaces of non-positive curvature, Springer Verlag, Berlin, 1999. MR1744486 (2000k:53038)

4. F. Bruhat and J. Tits, Groupes réductifs sur un corps local. I. Données radicielles valuées, Inst. Hautes Études Sci. Publ. Math. 41 (1972), 5-251. MR0327923 (48:6265)

5. C. E. Chidume, On the approximation of fixed points of nonexpansive mappings, Houston Journal of Mathematics 7 (1981), 345-355. MR640975 (83b:47063)

6. W. G. Dotson, On the Mann iterative process, Trans. Amer. Math. Soc. 149 (1970), 65-73. MR0257828 (41:2477)

7. M. Edelstein and R.C. O'Brien, Nonexpansive mappings, asymptotic regularity and successive approximations, J. London Math. Soc. 17 (1978), 547-554. MR500642 (80b:47074) 
8. J. Garcia-Falset, E. Llorens-Fuster, and S. Prus, The fixed point property for mappings admitting a center, Nonlinear Analysis 66 (2007), 1257-1274.

9. P. Gerhardy and U. Kohlenbach, Strongly uniform bounds from semi-constructive proofs, Ann. Pure Appl. Logic 141 (2006), 89-107. MR2229932

10. K. Gödel, Über eine bisher noch nicht benützte Erweiterung des finiten Standpunktes, Dialectica 12 (1958), 280-287. MR0102482 (21:1275)

11. K. Goebel and W. A. Kirk, Iteration processes for nonexpansive mappings, Topological Methods in Nonlinear Functional Analysis (S.P. Singh, S. Thomeier, and B. Watson, eds.), Contemporary Mathematics, vol. 21, AMS, Providence, R.I., 1983, pp. 115-123. MR729507 (85a:47059)

12. W. A. Howard, Hereditarily majorizable functionals of finite type, Metamathematical investigation of intuitionistic arithmetic and analysis (A.S. Troelstra, ed.), Springer LNM, vol. 344, Springer-Verlag, Berlin, 1973, pp. 454-461. MR0469712 (57:9493)

13. S. Ishikawa, Fixed points and iterations of a nonexpansive mapping in a Banach space, Proc. Amer. Math. Soc. 59 (1976), 65-71. MR0412909 (54:1030)

14. W. A. Kirk, Krasnosel'skii iteration process in hyperbolic spaces, Numer. Funct. Anal. Optimiz. 4 (1982), 371-381. MR673318 (84e:47067)

15. - Nonexpansive mappings and asymptotic regularity, Nonlinear Analysis 40 (2000), 323-332. MR1768416 (2001m:47116)

16. S.C. Kleene, Recursive functionals and quantifiers of finite types, I., Trans. Amer. Math. Soc. 91 (1959), 1-52. MR0102480 (21:1273)

17. U. Kohlenbach, A logical uniform boundedness principle for abstract metric and hyperbolic spaces, Electronic Notes in Theoretical Computer Science 165 (2006), 81-93. MR2321765

18. _ Effective uniform bounds from proofs in abstract functional analysis, CiE 2005. New Computational Paradigms: Changing Conceptions of What is Computable (B. Cooper, B. Loewe, and A. Sorbi, eds.), Springer Publisher, To appear.

19. E_ffective moduli from ineffective uniqueness proofs. An unwinding of de La Vallee Poussin's proof for Chebycheff approximation, Ann. Pure Appl. Logic 64 (1993), 27-94. MR1241250 (95b:03061)

20. _ Arithmetizing proofs in analysis, Logic Colloquium '96 (J.M. Larrazabal, D. Lascar, and G. Mints, eds.), Springer Lecture Notes in Logic, vol. 12, Springer-Verlag, Berlin, 1998, pp. 115-158. MR1674949 (2000j:03085)

21. , A quantitative version of a theorem due to Borwein-Reich-Shafrir, Numer. Funct. Anal. and Optimiz. 22 (2001), 641-656. MR1849571 (2002i:47076)

22. - On the computational content of the Krasnoselski and Ishikawa fixed point theorems, Proc. of the Fourth Workshop on Computability and Complexity in Analysis (J. Blanck, V. Brattka, and P. Hertling, eds.), volume 2064 of Springer Lecture Notes in Computer Science, 2001, pp. 119-145. MR1893075 (2003b:47090)

23. __ Uniform asymptotic regularity for Mann iterates, J. Math. Anal. Appl. 279 (2003), 531-544. MR1974043 (2004d:47098)

24. Some computational aspects of metric fixed point theory, Nonlinear Analysis $\mathbf{6 1}$, no.5 (2005), 823-837. MR2130066 (2005k:47119)

25. - Some logical metatheorems with applications in functional analysis, Trans. Amer. Math. Soc. 357 (2005), 89-128 (For some minor errata see the end of [9]). MR2098088 (2005h:03110)

26. U. Kohlenbach and B. Lambov, Bounds on iterations of asymptotically quasi-nonexpansive mappings, Proc. International Conference on Fixed Point Theory and Applications, Valencia 2003 (J.G. Falset, E.L. Fuster, and B. Sims, eds.), Yokohama Publishers, 2004, pp. 143-172. MR2144170 (2006b:47102)

27. U. Kohlenbach and L. Leustean, The approximate fixed point property in product spaces, Nonlinear Analysis 66 (2007), 806-818.

28. _ Mann iterates of directionally nonexpansive mappings in hyperbolic spaces, Abstract and Applied Analysis 8 (2003), 449-477. MR1983075 (2004e:47092)

29. U. Kohlenbach and P. Oliva, Proof mining: a systematic way of analyzing proofs in mathematics, Proc. Steklov Inst. Math 242 (2003), 136-164. MR2054493 (2005a:03111)

30. M.A. Krasnoselski, Two remarks on the method of successive approximation, Usp. Math. Nauk (N.S.) 10 (1955), 123-127 (Russian). MR0068119 (16:833a) 
31. L. Leustean, A quadratic rate of asympotic regularity for CAT(0)-spaces, J. Math. Anal. Appl. 325 (2007), 386-399. MR2273533

32 . Proof mining in R-trees and hyperbolic spaces, Electronic Notes in Theoretical Computer Science 165 (2006), 95-106.

33. H. Luckhardt, Extensional Gödel functional interpretation, Springer LNM, vol. 306, SpringerVerlag, Berlin, 1973. MR0337512 (49:2281)

34. H. V. Machado, A characterization of convex subsets of normed spaces, Kodai Math. Sem. Rep. 25 (1973), 307-320. MR0326359 (48:4703)

35. W. R. Mann, Mean value methods in iteration, Proc. Amer. Math. Soc. 4 (1953), 506-510. MR0054846 (14:988f)

36. A. G. O'Farrell, When uniformly-continuous implies bounded, Irish Math. Soc. Bulletin 53 (2004), 53-56. MR2117500

37. S. Reich and I. Shafrir, Nonexpansive iterations in hyperbolic spaces, Nonlinear Analysis, Theory, Methods and Applications 15 (1990), 537-558. MR1072312 (91k:47135)

38. I. Shafrir, The approximate fixed point property in Banach and hyperbolic spaces, Israel J. Math. 71 (1990), 211-223. MR1088815 (92b:47096)

39. B. Sims, Examples of fixed point free mappings, Handbook of Metric Fixed Point Theory (W.A. Kirk and B. Sims, eds.), Kluwer Academic Publishers, 2001, pp. 35-48. MR1904273 (2003f:47099)

40. C. Spector, Provably recursive functionals of analysis: a consistency proof of analysis by an extension of principles formulated in current intuitionistic mathematics, Proceedings of Symposia in Pure Mathematics (J.C.E. Dekker, ed.), vol. 5, AMS, Providence, R.I., 1962, pp. 1-27. MR0154801 (27:4745)

41. W. Takahashi, A convexity in metric space and nonexpansive mappings, I. Kodai Math. Sem. Rep. 22 (1970), 142-149. MR0267565 (42:2467)

Department of Mathematics, University of Oslo, Blindern, N-0316 Oslo, Norway

Department of Mathematics, Technische Universität Darmstadt, SchlossgartenSTRASSE 7, D-64289 Darmstadt, Germany 